\title{
Search for lepton-flavor-violating decays of the $Z$ boson into a $\tau$ lepton and a light lepton with the ATLAS detector
}

\author{
M. Aaboud et al. \\ (ATLAS Collaboration)
}

(Received 26 April 2018; published 14 November 2018)

\begin{abstract}
Direct searches for lepton flavor violation in decays of the $Z$ boson with the ATLAS detector at the LHC are presented. Decays of the $Z$ boson into an electron or muon and a hadronically decaying $\tau$ lepton are considered. The searches are based on a data sample of proton-proton collisions collected by the ATLAS detector in 2015 and 2016, corresponding to an integrated luminosity of $36.1 \mathrm{fb}^{-1}$ at a center-of-mass energy of $\sqrt{s}=13 \mathrm{TeV}$. No statistically significant excess of events above the expected background is observed, and upper limits on the branching ratios of lepton-flavor-violating decays are set at the 95\% confidence level: $\mathcal{B}(Z \rightarrow e \tau)<5.8 \times 10^{-5}$ and $\mathcal{B}(Z \rightarrow \mu \tau)<2.4 \times 10^{-5}$. This is the first limit on $\mathcal{B}(Z \rightarrow e \tau)$ with ATLAS data. The upper limit on $\mathcal{B}(Z \rightarrow \mu \tau)$ is combined with a previous ATLAS result based on $20.3 \mathrm{fb}^{-1}$ of proton-proton collision data at a center-of-mass energy of $\sqrt{s}=8 \mathrm{TeV}$ and the combined upper limit at $95 \%$ confidence level is $\mathcal{B}(Z \rightarrow \mu \tau)<1.3 \times 10^{-5}$.
\end{abstract}

DOI: 10.1103/PhysRevD.98.092010

\section{INTRODUCTION}

One of the main goals of the physics program of the Large Hadron Collider (LHC) at CERN is to discover physics beyond the Standard Model (SM). The observation of lepton flavor violation in decays of the $Z$ boson into a pair of leptons of different flavors would give a clear indication for new physics. These decays can occur within the SM only via neutrino oscillations and would have a rate too small to be detected [1]. This paper presents searches by the ATLAS Collaboration for the decays of the $Z$ boson into a $\tau$ lepton and an electron or a muon, hereafter referred to as a light lepton or $\ell$. Only final states with a hadronically decaying $\tau$ lepton are considered.

Lepton-flavor-violating (LFV) $Z$ boson decays are predicted by models with heavy neutrinos [2], extended gauge models [3] and supersymmetry [4]. The most stringent bounds on such decays with a $\tau$ lepton in the final state are set by the LEP experiments: $\mathcal{B}(Z \rightarrow e \tau)<9.8 \times 10^{-6}[5]$ and $\mathcal{B}(Z \rightarrow \mu \tau)<1.2 \times 10^{-5}[6]$ at $95 \%$ confidence level (C.L.). The ATLAS experiment has set the upper limit $\mathcal{B}(Z \rightarrow \mu \tau)<1.7 \times 10^{-5}$ at $95 \%$ C.L. [7] by analyzing $20.3 \mathrm{fb}^{-1}$ of proton-proton collisions at a center-of-mass energy of $8 \mathrm{TeV}$. There are no previously published limits

*Full author list given at the end of the article.

Published by the American Physical Society under the terms of the Creative Commons Attribution 4.0 International license. Further distribution of this work must maintain attribution to the author(s) and the published article's title, journal citation, and DOI. Funded by SCOAP ${ }^{3}$. on $\mathcal{B}(Z \rightarrow e \tau)$ with ATLAS data. Regarding the LFV $Z \rightarrow$ $e \mu$ decays, at the LHC the CMS experiment set the upper bound at $\mathcal{B}(Z \rightarrow e \mu)<7.3 \times 10^{-7}$ at $95 \%$ C.L. [8]. The ATLAS experiment obtained a similar result with the upper bound set at $\mathcal{B}(Z \rightarrow e \mu)<7.5 \times 10^{-7}$ at $95 \%$ C.L. [9].

The searches for LFV $Z$ decays presented in this paper use a data sample of proton-proton collisions collected at a center-of-mass energy of $\sqrt{s}=13 \mathrm{TeV}$ with the ATLAS detector at the LHC. These data correspond to an integrated luminosity of $36.1 \mathrm{fb}^{-1}$. The signal model used assumes unpolarized $\tau$ leptons. Events are classified using neural networks, and the output distribution is used in a template fit to data to extract the $Z$ boson lepton-flavorviolating branching ratios, or otherwise set upper limits on these values. The major backgrounds to the search are reducible backgrounds such as $W+$ jets, top-quark pair production and $Z \rightarrow \ell \ell$, and the irreducible background $Z \rightarrow \tau \tau \rightarrow \ell+$ hadrons $+3 \nu$. Reducible backgrounds from events with a quark- or gluon-initiated jet misidentified as a hadronically decaying $\tau$ lepton, so-called "fakes," are estimated via a data-driven method. The reducible backgrounds from $Z \rightarrow \ell \ell$, where one light lepton fakes a hadronic $\tau$-lepton decay signature, are estimated using simulation. An event selection specifically designed to reduce the contribution from this background is applied. The shape of the template for the irreducible background from $Z \rightarrow \tau \tau$ is estimated via simulations and its magnitude is determined in the fit to data.

The results of the search for the LFV $Z \rightarrow \mu \tau$ decays presented in this paper are combined with the previous ATLAS results based on $8 \mathrm{TeV}$ data. 
This paper is structured as follows. Section II briefly describes the ATLAS detector and the reconstruction of the detected particles. Section III details the data sample and the simulations used in the analysis. Section IV describes the event selection and classification criteria. Section V discusses the methodology used to estimate the yield of events from background sources, and Sec. VI lists the experimental and theoretical systematic uncertainties affecting the analysis. The statistical interpretation of the observed data and the results are presented in Sec. VII. The combination of the result in the $Z \rightarrow \mu \tau$ channel with the previous ATLAS result from $8 \mathrm{TeV}$ data is also presented. Finally, Sec. VIII summarizes the analysis.

\section{THE ATLAS DETECTOR AND OBJECT RECONSTRUCTION}

The ATLAS detector ${ }^{1}[10]$ at the LHC is a multipurpose particle detector with a forward-backward symmetric cylindrical geometry and a nearly $4 \pi$ coverage in solid angle. It consists of an inner tracking detector, electromagnetic and hadronic calorimeters, and a muon spectrometer. The inner detector (ID), immersed in a $2 \mathrm{~T}$ axial magnetic field provided by a thin superconducting solenoid, includes silicon pixel and microstrip detectors, which provide precision tracking in the pseudorapidity range $|\eta|<2.5$, and a transition-radiation tracker providing additional tracking and information for electron identification for $|\eta|<2.0$. For the $\sqrt{s}=13 \mathrm{TeV}$ data-taking period, the ID was upgraded with a silicon-pixel insertable B-layer [11] that provides additional tracking information closer to the interaction point. The solenoid is surrounded by sampling calorimeters: a lead/liquid-argon (LAr) electromagnetic calorimeter covering the region $|\eta|<3.2$, a hadronic calorimeter with a steel/scintillator-tile barrel section for $|\eta|<1.7$ and two copper/LAr end caps for $1.5<|\eta|<3.2$. The forward region is covered by additional LAr calorimeters with coarser granularity up to $|\eta|=4$.9. The calorimeter is surrounded by the muon spectrometer, which is based on three large superconducting toroid magnets each containing eight coils. Precise momentum measurements for muons with pseudorapidity up to $|\eta|=2.7$ are provided by three layers of tracking chambers. The muon spectrometer also includes separate trigger chambers covering $|\eta|<2.4$. A two-level trigger

\footnotetext{
${ }^{1}$ ATLAS uses a right-handed coordinate system with its origin at the nominal interaction point (IP) in the center of the detector and the $z$ axis along the beam pipe. The $x$ axis points from the IP to the center of the LHC ring, and the $y$ axis points upward. Cylindrical coordinates $(r, \phi)$ are used in the transverse plane, with $\phi$ as the azimuthal angle around the beam pipe. The pseudorapidity is defined in terms of the polar angle $\theta$ as $\eta=-\ln \tan (\theta / 2)$. The transverse momentum and the transverse energy are defined as $p_{\mathrm{T}}=p \sin \theta$ and $E_{\mathrm{T}}=E \sin \theta$, respectively. The distance $\Delta R$ in $\eta-\phi$ space is defined as $\Delta R=$ $\sqrt{(\Delta \eta)^{2}+(\Delta \phi)^{2}}$.
}

system [12] was used during the $\sqrt{s}=13$ data-taking period. The first-level trigger (L1) is implemented in hardware and uses a subset of the detector information. This is followed by a software-based level which runs algorithms similar to the offline reconstruction software, reducing the event rate to approximately $1 \mathrm{kHz}$ on average from the maximum $\mathrm{L} 1$ rate of $100 \mathrm{kHz}$.

Electron candidates are reconstructed from energy deposits in the electromagnetic calorimeter which are matched to a charged-particle track measured in the inner detector. These candidates are required to satisfy "medium" likelihood-based identification criteria [13], to have a transverse momentum $p_{\mathrm{T}}>30 \mathrm{GeV}$ and to be in the acceptance region $|\eta|<2.47$ of the inner detector. Candidates in the transition region $1.37<|\eta|<1.52$ between the barrel and end cap calorimeters are excluded.

Muon candidates are reconstructed from track segments in the muon spectrometer which are matched to tracks reconstructed in the inner detector which satisfy $|\eta|<2.5$. The matched tracks are re-fitted using the complete track information from both detector subsystems. Muon candidates are required to satisfy "medium" identification criteria [14] and to have a $p_{\mathrm{T}}>30 \mathrm{GeV}$.

Isolation criteria are applied to both the electrons and muons using calorimeter- and track-based information. The calorimeter-based variables are corrected for the average energy contributions from additional proton-proton interactions in the same and neighboring bunch crossings ("pileup") [13]. A standard working point is used, where a $90 \%$ efficiency is obtained for leptons with $p_{\mathrm{T}}=25 \mathrm{GeV}$, rising to $99 \%$ efficiency at $p_{\mathrm{T}}=60 \mathrm{GeV}$ in $Z \rightarrow \ell \ell$ events. Events with electrons or muons that fail the isolation criteria are used to estimate background contributions, as described in Sec. V.

Topological clusters of energy deposits in the calorimeter are reconstructed into jets with the anti- $k_{t}$ algorithm [15] and radius parameter $R=0.4$ using the FASTJET software package [16]. Energy deposits from pileup are subtracted using an average pileup energy density and the jet area. Jets are then calibrated as described in Ref. [17]. Jet candidates are required to have $p_{\mathrm{T}}>20 \mathrm{GeV}$ and $|\eta|<2.5$. To further reduce the effect of pileup, a jet vertex tagger (JVT) algorithm is used for jets with $p_{\mathrm{T}}<60 \mathrm{GeV}$ and $|\eta|<2.4$. The JVT algorithm employs a multivariate technique based on jet energy, vertexing, and tracking variables in order to determine the likelihood that jets originate from or are heavily contaminated by pileup [18].

In order to identify jets containing $b$-hadrons ( $b$-jets), a multivariate algorithm is used that depends on the presence of tracks with a large impact parameter with respect to the primary vertex [19], on the presence of displaced secondary vertices, and on the reconstructed flight paths of $b$ - and $c$-hadrons associated with the jet [20]. Using this algorithm, jets are $b$-tagged if they satisfy criteria of a standard working point, which provides a $b$-jet efficiency of $77 \%$ 
and a light-jet rejection rate of about 134 in simulated $t \bar{t}$ events

Hadronic $\tau$-lepton decays result in a neutrino and a set of visible decay products $\left(\tau_{\text {had-vis }}\right)$, typically one or three charged pions and up to two neutral pions [21]. The reconstruction of the visible decay products [22] is seeded by jets. Selected $\tau_{\text {had-vis }}$ candidates are required to have $p_{\mathrm{T}}>20 \mathrm{GeV},|\eta|<2.5$ excluding $1.37<|\eta|<1.52$, one (1-prong) or three (3-prong) associated tracks with $p_{\mathrm{T}}>1 \mathrm{GeV}$, and an electric charge of \pm 1 . A boosted decision tree (BDT) identification procedure that is based on calorimetric shower shapes and tracking information is used to discriminate $\tau$-lepton decays from jet backgrounds $[23,24]$. All events used in this analysis must have a $\tau_{\text {had-vis }}$ candidate that passes the "loose" identification working point. For events in the signal region, the $\tau_{\text {had-vis }}$ candidate must satisfy the "tight" identification criterion. Selected events that are not in the signal region are used to estimate backgrounds (Sec. V). The combined reconstruction and identification efficiencies for loose and tight criteria are $60 \%(50 \%)$ and $45 \%$ (30\%) for 1-prong (3-prong) hadronic $\tau$-lepton decays, and are independent of the $\tau_{\text {had-vis }} p_{\mathrm{T}}$ and the number of pileup interactions. To reduce the number of muons misidentified as $\tau_{\text {had-vis, }}$ a $\tau_{\text {had-vis }}$ candidate is excluded if it is within $\Delta R=0.2$ of a reconstructed muon with $p_{\mathrm{T}}>2 \mathrm{GeV}$. An additional BDT, denoted hereafter by eBDT, is used to reduce the number of electrons misidentified as $\tau_{\text {had-vis, }}$, providing $85 \%$ (95\%) efficiency for 1-prong (3-prong) hadronic $\tau$-lepton decays. The leading- $p_{\mathrm{T}}$ candidate is selected as the $\tau_{\text {had-vis }}$ candidate, while any other candidates are considered to be jets.

To avoid potential ambiguities among objects, light lepton and $\tau_{\text {had-vis }}$ candidates are required to be separated from each other and from jets in the following order: (a) jets within $\Delta R=0.2$ of selected $\tau_{\text {had-vis }}$ candidates are excluded, (b) jets within $\Delta R=0.4$ of an electron or a muon are excluded, (c) any $\tau_{\text {had-vis }}$ within $\Delta R=0.2$ of an electron or a muon is excluded, and (d) electrons within $\Delta R=0.2$ of a muon are excluded.

The missing transverse momentum, with magnitude $E_{\mathrm{T}}^{\text {miss }}$, is calculated as the negative vectorial sum of the transverse momenta of all fully reconstructed and calibrated ("hard") physics objects and inner-detector tracks that originate from the hard-scattering vertex but are not matched to a reconstructed object ("soft term") [25]. The soft term is an important contribution for improving both the $E_{\mathrm{T}}^{\text {miss }}$ scale and its resolution.

\section{DATA AND SIMULATED EVENT SAMPLES}

This search analyzes proton-proton collisions recorded by the ATLAS detector at the LHC during 2015 and 2016 at a center-of-mass energy of $\sqrt{s}=13 \mathrm{TeV}$. The data correspond to a total integrated luminosity of $36.1 \mathrm{fb}^{-1}$ after requiring that all relevant components of the ATLAS detector were in good working condition during data collection. The uncertainty in the combined 2015 and 2016 integrated luminosity is $2.1 \%$. It was estimated following a methodology similar to the one described in Ref. [26]. The events considered for the $e \tau(\mu \tau)$ channel were selected by single-lepton triggers which require the presence of at least one electron (muon) candidate with transverse momentum above $24 \mathrm{GeV}(20 \mathrm{GeV})$ in 2015 data and $26 \mathrm{GeV}(26 \mathrm{GeV})$ in 2016 data. These triggers apply isolation criteria for electron (muon) candidates with $p_{\mathrm{T}}$ below $60 \mathrm{GeV}$ (40 GeV in 2015 and $50 \mathrm{GeV}$ in 2016). These isolation requirements are looser than the ones applied offline in the light-lepton selections used in this analysis.

Simulated Monte Carlo (MC) samples are used to predict the $Z / \gamma^{*} \rightarrow \ell \tau$ signal and the background contributions from $Z / \gamma^{*}+$ jets, $W+$ jets, $t \bar{t}$, single top-quark, Higgs boson and diboson ( $W W, W Z$ and $Z Z$ ) production.

Signal samples were simulated using PYTHIA 8.186 [27] with the NNPDF2.3 parton distribution function (PDF) set [28] and a set of tuned parameters called the A14 tune [29]. The lepton-flavor-violating $Z / \gamma^{*}$ decay was modeled assuming unpolarized $\tau$ leptons in the final state. To ensure that both the hypothetical signal $Z \rightarrow \ell \tau$ and the main background $Z / \gamma^{*} \rightarrow \tau \tau$ are normalized to the same production cross section, event weights computed as a function of the true boson transverse momentum are applied to the signal events to match the more accurate modeling of the $Z / \gamma^{*}$ production in the $Z / \gamma^{*} \rightarrow \tau \tau$ simulation described in the following. After this reweighting procedure, the signal events, together with the $Z / \gamma^{*} \rightarrow \tau \tau$ events, are normalized to the $Z / \gamma^{*}$ production cross section determined from data in the template fit described in Sec. VII. Therefore, the analysis is independent of the theoretical uncertainty in the $Z / \gamma^{*}$ production cross section. The SM value of this cross section is $2.1 \mathrm{nb}$, calculated at NNLO accuracy [30].

The production of $Z / \gamma^{*} \rightarrow \tau \tau$ events was simulated with SHERPA 2.2.1 [31]. The NNPDF 3.0 NNLO PDF set [32] was used for both the matrix element calculation and the dedicated parton-shower tuning developed by the authors of SHERPA. The event generation utilized COMIX [33] and OpenLoops [34] for the matrix element calculation, which was then matched to the SHERPA parton shower using the ME+PS@NLO prescription [35]. The matrix elements were calculated for up to two additional partons at NLO and for three and four partons at LO in QCD. As stated above, the normalization of this background process, together with the signal events, is determined in a fit to data.

The $Z / \gamma^{*} \rightarrow \mu \mu$, ee events were simulated with Powheg-Box [36-38] using the CT10 PDF set [39] and the AZNLO tune [40], and interfaced to PYTHIA 8.186. The normalization of the $Z / \gamma^{*} \rightarrow \mu \mu$, ee events is determined from data in a dedicated region enhanced in $Z \rightarrow \mu \mu$ events (Sec. V) as a function of the reconstructed transverse momentum of the $Z / \gamma^{*}$ boson. 
The other simulated processes account for only a small fraction (less than 0.3\%) of the background events. Samples of $W(\rightarrow \tau \nu)+$ jets events were simulated with SHERPA 2.2.1. Events with a top-quark pair or a single top quark produced via electroweak $t$-channel, $s$-channel and $W t$-channel processes were simulated with PowHEG-Box using the CT10 PDF set. The parton shower, fragmentation and underlying event were simulated using PYTHIA 6.428 [41] with the Perugia 2012 tune [42]. EvTGEN [43] was used to decay bottom and charm hadrons. Diboson processes were simulated with SHERPA 2.1.0 with the CT10 PDF set. Higgs boson events, $H \rightarrow W W, \tau \tau, \ell \ell$, produced via gluon-gluon fusion and vector-boson fusion were simulated with PowHEg-Box.

Simulated minimum-bias events were overlaid on all simulated samples to include the effect of pileup. These minimum-bias events were generated with PYтHIA 8.186, using the A2 tune [44] and the MSTW2008LO PDF set [45]. Each simulated event was processed using the GEANTbased ATLAS detector simulation [46,47] and the same event reconstruction algorithms used for the data. Reconstruction and identification efficiencies, as well as energy calibrations for all selected objects in simulated events, are corrected to match those measured in data.

\section{EVENT SELECTION AND CLASSIFICATION}

Of the events satisfying the trigger and the quality criteria described in Sec. III, the events selected in this analysis are required to contain exactly one isolated electron or muon that is geometrically matched to the object that fired the trigger, and no additional light leptons. These events must also contain at least one $\tau_{\text {had-vis }}$ candidate that passes the tight identification. The isolated light lepton and the $\tau_{\text {had-vis }}$ candidate are required to have opposite charge, $q_{\ell} q_{\tau_{\text {had-vis }}}=-1$. Events with one or more $b$-tagged jets are removed to reject background events with a topquark pair or a singly produced top quark. To reduce the $Z \rightarrow \ell \ell$ background, events with 1-prong $\tau_{\text {had-vis }}$

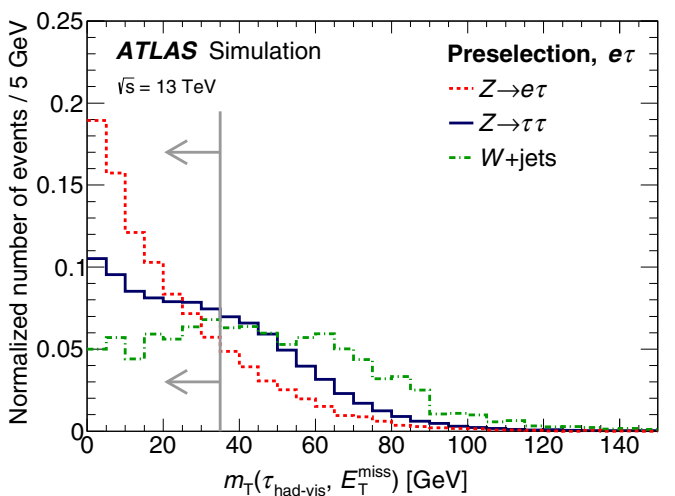

candidates that satisfy $\left|\eta\left(\tau_{\text {had-vis }}\right)\right|>2.2$ for the $e \tau$ channel or $\left|\eta\left(\tau_{\text {had-vis }}\right)\right|<0.1$ for the $\mu \tau$ channel are rejected. These regions of the detector are excluded because they are insufficiently instrumented and therefore affected by higher $\ell \rightarrow \tau$ misreconstruction and misidentification rates. The selection described here, denoted hereafter to as preselection, defines the sample of events used for the training of the neural network.

Further kinematic selections are applied to define the sample of events in the "signal region" (SR) which are used in the final template fit. Orthogonal sets of events in the socalled "calibration regions" (CR) are defined by inverting some of the preselection or SR selection requirements and used to estimate background contributions in the SR, as described in Sec. V.

Events accepted in the SR must satisfy the preselection and the following selections. The transverse mass,

$$
\begin{aligned}
& m_{\mathrm{T}}\left(\tau_{\text {had-vis }}, E_{\mathrm{T}}^{\mathrm{miss}}\right) \\
& \equiv \sqrt{2 p_{\mathrm{T}}\left(\tau_{\text {had-vis }}\right) E_{\mathrm{T}}^{\text {miss }}\left[1-\cos \left(\Delta \phi\left(\tau_{\text {had-vis }}, E_{\mathrm{T}}^{\text {miss }}\right)\right)\right]},
\end{aligned}
$$

is required to be smaller than $35(30) \mathrm{GeV}$ in the $e \tau(\mu \tau)$ channel. Signal events are expected to have the missing transverse momentum from the neutrino in a direction close to the $\tau_{\text {had-vis }}$ candidate, resulting in small $m_{\mathrm{T}}\left(\tau_{\text {had-vis }}, E_{\mathrm{T}}^{\text {miss }}\right)$ values. The $W(\rightarrow \ell \nu / \tau \nu)+$ jets events and some of the $Z / \gamma^{*} \rightarrow \tau \tau$ events have instead higher $m_{\mathrm{T}}\left(\tau_{\text {had-vis }}, E_{\mathrm{T}}^{\mathrm{miss}}\right)$ values. This selection allows the definition of a CR that is dominated by $W+$ jets events, which are the major contribution to fakes. The selection is illustrated in Fig. 1. In events with a 1-prong $\tau_{\text {had-vis }}$ candidate, an additional selection is applied to further reduce the $Z \rightarrow \ell \ell$ background. In most of these events, the momentum of the track matched to the 1-prong $\tau_{\text {had-vis }}$ candidate corresponds to the original momentum of the light lepton misidentified as $\tau_{\text {had-vis }}$, while the energy deposited in the calorimeter and used to estimate the energy of the $\tau_{\text {had-vis }}$ originates from

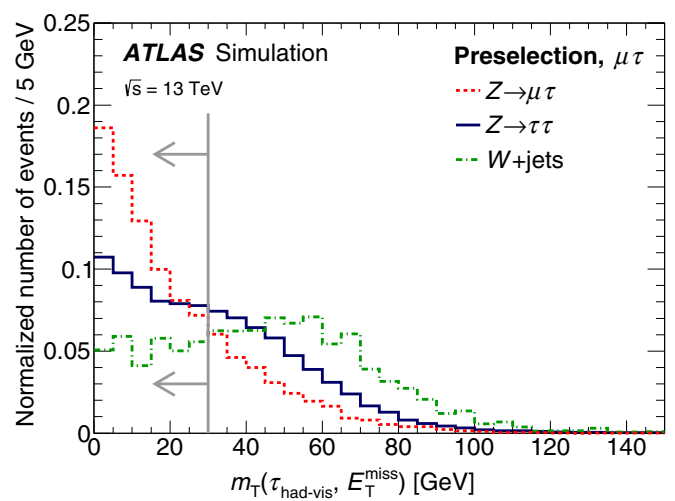

FIG. 1. Expected distributions of $m_{\mathrm{T}}\left(\tau_{\text {had-vis }}, E_{\mathrm{T}}^{\mathrm{miss}}\right)$ in $Z / \gamma^{*} \rightarrow \tau \tau, W(\rightarrow \ell \nu / \tau \nu)+$ jets and signal events in the $e \tau$ (left) and $\mu \tau$ (right) channels after preselection requirements. The $Z / \gamma^{*} \rightarrow \tau \tau$ and $W(\rightarrow \ell \nu / \tau \nu)+$ jets distributions also include the contributions to fakes from the corresponding processes as predicted by MC simulations. All distributions are normalized to unity. 
radiation (light-lepton bremsstrahlung) or other sources. Therefore, events in which the invariant mass of the $\tau_{\text {had-vis }}$ track and the light lepton $(m($ track, $\ell))$ is compatible with the $Z$ boson mass are rejected. In particular, events with a 1-prong $\tau_{\text {had-vis }}$ candidate are accepted when $m($ track, $\ell)<$ $84 \mathrm{GeV}$ or $m($ track, $\ell)>105 \mathrm{GeV}$ if $\left|\eta\left(\tau_{\text {had-vis }}\right)\right|<2.0$, and when $m($ track, $\ell)<80 \mathrm{GeV}$ or $m($ track, $\ell)>105 \mathrm{GeV}$ if $\left|\eta\left(\tau_{\text {had-vis }}\right)\right|>2.0$. A wider range in $m($ track, $\ell$ ) is rejected at high $\left|\eta\left(\tau_{\text {had-vis }}\right)\right|$ because of the smaller signal contribution and the higher $Z \rightarrow \ell \ell$ background rate. Moreover, events in which the invariant mass of the 1-prong $\tau_{\text {had-vis }}$ candidate and the light lepton satisfies $80 \mathrm{GeV}<m\left(\tau_{\text {had-vis }}, \ell\right)<100 \mathrm{GeV}$ are required to have $m($ track, $\ell)>40 \mathrm{GeV}$. These selections have been optimized in the $m\left(\tau_{\text {had-vis }}, \ell\right)-m($ track, $\ell)$ plane to specifically reject the $Z \rightarrow \ell \ell$ background events at a small acceptance loss for signal. The impact of these selections is illustrated in Fig. 2.

The signal selection efficiency in the SR is $3.2 \%$ (3.5\%) for the $e \tau(\mu \tau)$ channel. The $Z \rightarrow \tau \tau, Z \rightarrow \ell \ell$ and $W \rightarrow$ $\ell \nu+$ jets background selection efficiencies are, including
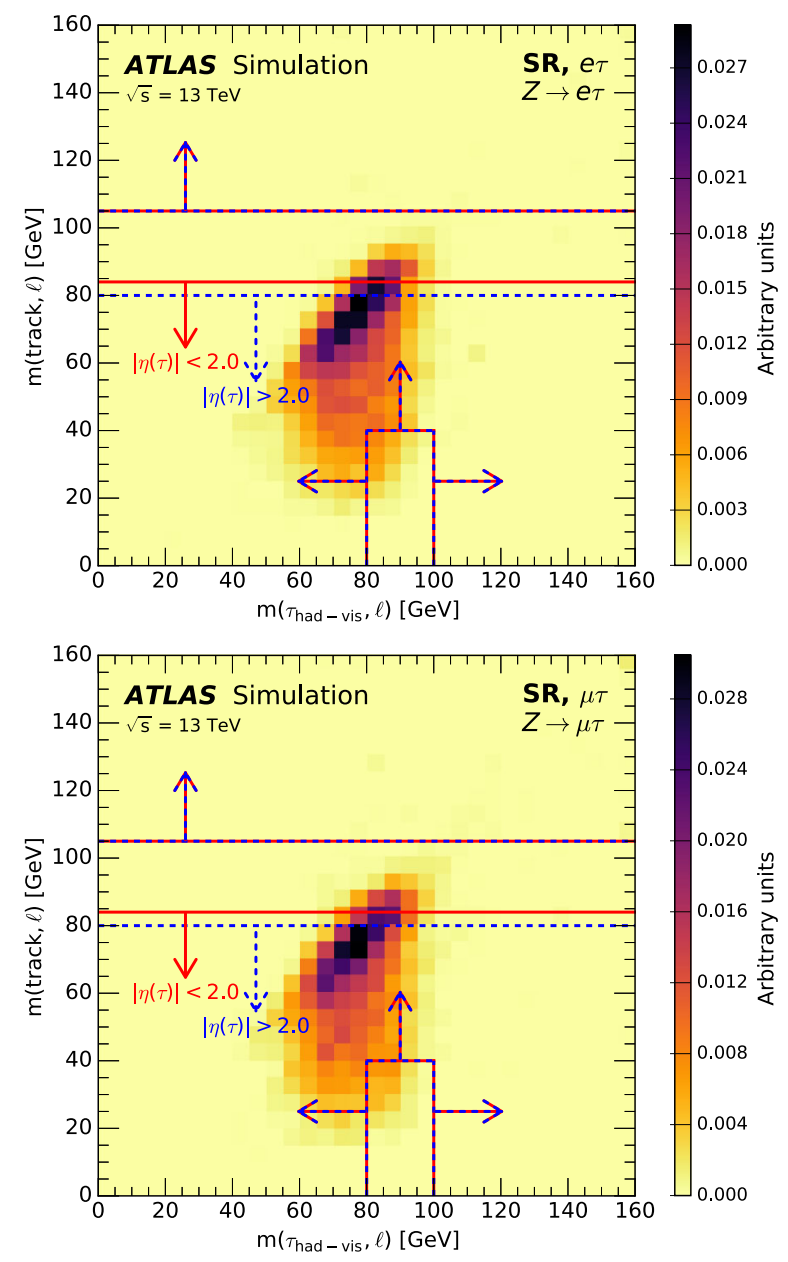

their contributions to fakes as predicted by $\mathrm{MC}$ simulations, $7.1 \times 10^{-3}\left(6.5 \times 10^{-3}\right), \quad 9.4 \times 10^{-5} \quad\left(5.9 \times 10^{-5}\right)$ and $2.6 \times 10^{-5}\left(2.9 \times 10^{-5}\right)$ respectively. A summary of the event selection criteria is given in Table I.

Events accepted in the SR are classified using neural networks (NNs) trained to discriminate $Z \rightarrow \ell \tau$ signal from $Z \rightarrow \tau \tau, Z \rightarrow \ell \ell$ and $W \rightarrow \ell \nu+$ jets background events. The classification is based on event kinematic properties that are extracted by the NN from the reconstructed momenta of the selected particles, as well as from other event variables. The NN achieves good performance using low-level variables, such as the particle momentum components, due to the network's capability to build non-linear relations between input variables.

Three types of NN classifiers, "Z", "Zll" and "W", are trained to distinguish signal from $Z \rightarrow \tau \tau, Z \rightarrow \ell \ell$ and $W \rightarrow \ell \nu$ backgrounds, respectively. These classifiers are trained separately in the $e \tau$ and $\mu \tau$ channels because of the different detector acceptances, but combine 1-prong and 3 -prong $\tau_{\text {had-vis }}$ candidates. Simulated events passing the preselection (Table I) are used to train, optimize and
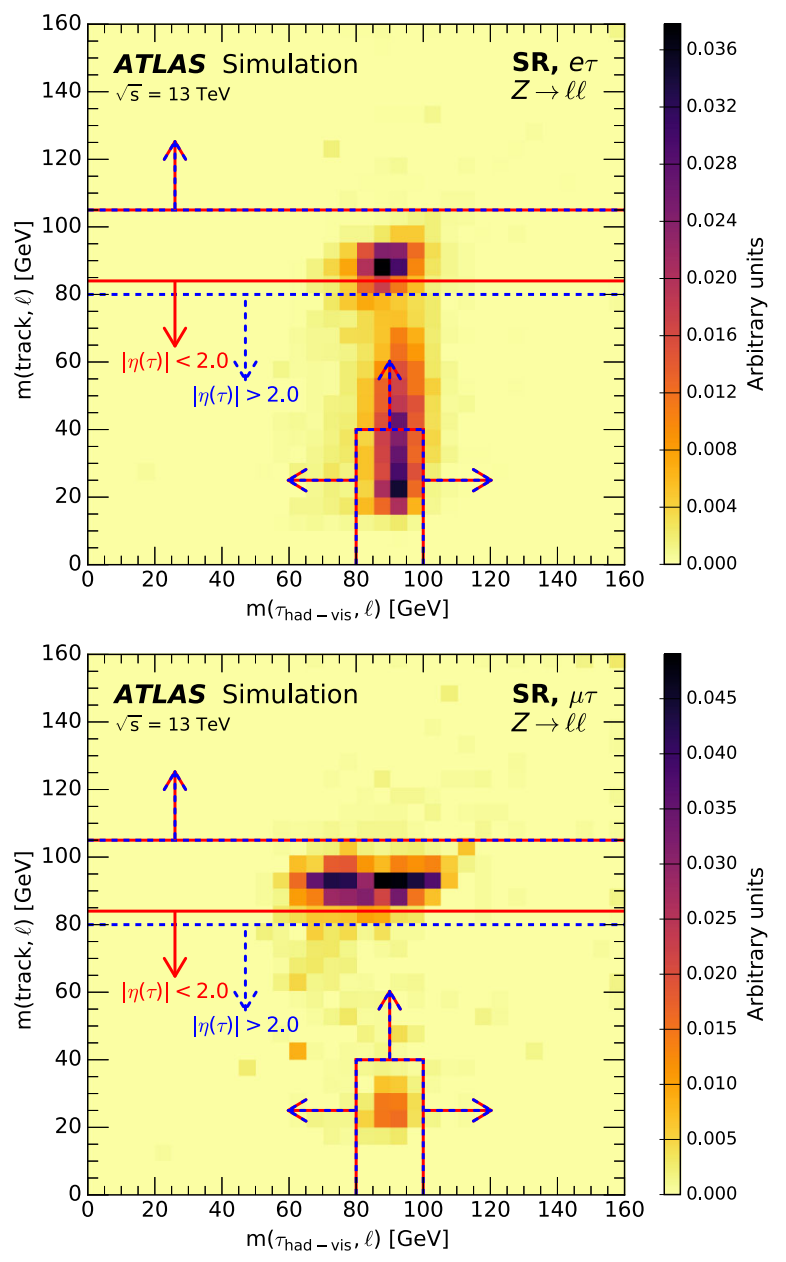

FIG. 2. Expected distributions of $m($ track, $\ell)$ versus $m\left(\tau_{\text {had-vis }}, \ell\right)$ in signal (left) and $Z \rightarrow \ell \ell$ (right) events with 1-prong $\tau_{\text {had-vis }}$ candidates in the $e \tau$ (top) and $\mu \tau$ (bottom) channels after the SR selection except for the cuts on these two variables (Table I). 
TABLE I. Overview of the event selection. More details are given in Secs. II and IV.

Preselection

Signal region one isolated tight light lepton with $p_{\mathrm{T}}>30 \mathrm{GeV}$ matched to a lepton selected at trigger level leading $\tau_{\text {had-vis }}$ with $p_{\mathrm{T}}>20 \mathrm{GeV}, N_{\tau}^{\text {tracks }}=1$ or 3 and passing tight identification if $N_{\tau}^{\text {tracks }}=1: 0.0(0.1)<\left|\eta_{\tau}\right|<1.37$ or $1.52<\left|\eta_{\tau}\right|<2.2(2.5)$ in $e \tau(\mu \tau)$ events

if $N_{\tau}^{\text {tracks }}=3: 0.0<\left|\eta_{\tau}\right|<1.37$ or $1.52<\left|\eta_{\tau}\right|<2.5$

$q_{\ell} \times q_{\tau}=-1$

no $b$-jet, no additional light lepton

$m_{\mathrm{T}}\left(\tau_{\text {had-vis }}, E_{\mathrm{T}}^{\mathrm{miss}}\right)<35(30) \mathrm{GeV}$ in $e \tau(\mu \tau)$ events

if $N_{\tau}^{\text {tracks }}=1$ and $\left|\eta_{\tau}\right|<2.0: m($ track, $\ell)<84 \mathrm{GeV}$ or $m($ track, $\ell)>105 \mathrm{GeV}$

if $N_{\tau}^{\text {tracks }}=1$ and $\left|\eta_{\tau}\right|>2.0: m($ track, $\ell)<80 \mathrm{GeV}$ or $m($ track, $\ell)>105 \mathrm{GeV}$

if $N_{\tau}^{\text {tracks }}=1$ and $80<m\left(\tau_{\text {had-vis }}, \ell\right)<100 \mathrm{GeV}: m($ track, $\ell)>40 \mathrm{GeV}$ validate the classifiers. In order to increase the size of the available training samples for $Z \rightarrow \ell \tau$ and $Z \rightarrow \tau \tau$ processes with a true hadronic $\tau$-lepton decay, all events with a $\tau_{\text {had-vis }}$ candidate that passes the loose identification are used. Moreover, in the events used for the Zll classifiers, the misreconstructed $\tau_{\text {had-vis }}$ is required to be either a true muon or electron. With these requirements, about 40000 signal events, $200000 Z \rightarrow \tau \tau$ events and $80000 \mathrm{~W} \rightarrow \ell \nu$ events are used for training in each channel. For $Z \rightarrow \ell \ell$, about 30000 events are used in the $e \tau$ channel and only 5000 events in the $\mu \tau$ channel. The limited number of $Z \rightarrow \mu \mu$ events is due to the low $\mu \rightarrow \tau$ misreconstruction rate, and leads to poor classification power for the Zll NN in the $\mu \tau$ channel. However, the $Z \rightarrow \mu \mu$ background is effectively reduced by the selection on $m(\operatorname{track}, \ell)$ and $m\left(\tau_{\text {had-vis }}, \ell\right)$ described earlier.

The input variables common to all the classifiers are the light lepton, $\tau_{\text {had-vis }}$ and $E_{\mathrm{T}}^{\text {miss }}$ momentum components, assuming vanishing masses; the collinear mass $m_{\text {coll }}$, defined as the invariant mass of the $\ell-\tau_{\text {had-vis }}-\nu$ system, where $\nu$ is the neutrino from the $\tau$ decay, which is assumed to have a momentum that is equal in the transverse plane to the measured $E_{\mathrm{T}}^{\text {miss }}$ and collinear in $\eta$ with the $\tau_{\text {had-vis }}$ candidate; and $\Delta \alpha[48]$ :

$$
\Delta \alpha=\frac{1}{2} \frac{m_{Z}^{2}-m_{\tau}^{2}}{p\left(\tau_{\text {had-vis }}\right) \cdot p(\ell)}-\frac{p_{\mathrm{T}}(\ell)}{p_{\mathrm{T}}\left(\tau_{\text {had-vis }}\right)},
$$

where $p\left(\tau_{\text {had-vis }}\right)$ and $p(\ell)$ are the four-momenta of the $\tau_{\text {had-vis }}$ and the light-lepton candidates respectively, and the rest masses $m_{Z}$ and $m_{\tau}$ take on values reported by the Particle Data Group [21]. The variable $\Delta \alpha$ helps to discriminate signal events, expected to be around $\Delta \alpha=0$, from $Z \rightarrow \tau \tau$ events, where $\Delta \alpha$ is negative due to the presence of additional neutrinos. Even though not specifically targeted by this variable, $Z \rightarrow \ell \ell$ and $W \rightarrow \ell \nu$ events tend to be at vanishing and positive values of $\Delta \alpha$, respectively, as shown later in Figs. 5-8. The invariant mass $m\left(\ell, \tau_{\text {had-vis }}\right)$ is also used in the Zll classifier. In the limit of very large training statistics, the light lepton, $\tau_{\text {had-vis }}$ and $E_{\mathrm{T}}^{\text {miss }}$ momentum components would be sufficient for the $\mathrm{NN}$ to learn the full event kinematics. However, with the available training samples, the high-level variables $m_{\text {coll }}$, $\Delta \alpha$ and $m\left(\ell, \tau_{\text {had-vis }}\right)$ were found to be able to improve the $\mathrm{NN}$ classification power and were therefore included among the NN inputs.

The NN inputs are preprocessed to harmonize their magnitudes and to remove known symmetries as is required for optimal training. The preprocessing consists of the following steps:

(1) Boost: after computing $m_{\text {coll }}, \Delta \alpha$ and $p^{\text {tot }}=p(\ell)+$ $p\left(\tau_{\text {had-vis }}\right)+E_{\mathrm{T}}^{\text {miss }}$ in the lab frame, the light lepton, $\tau_{\text {had-vis }}$ and $E_{\mathrm{T}}^{\text {miss }}$ momenta are boosted to the frame in which their total momentum vanishes. The longitudinal component of the three-momentum of $E_{\mathrm{T}}^{\text {miss }}$ is zero in the lab frame.

(2) Rotation: the light lepton, $\tau_{\text {had-vis }}$ and $E_{\mathrm{T}}^{\text {miss }}$ momenta are first rotated so that the three-momentum of the light lepton is along the positive $z$ axis. A second rotation about the $z$ axis is applied so that the $\tau_{\text {had-vis }}$ momentum has a vanishing component on the $y$ axis.

(3) "Feature scaling": each input variable is scaled by subtracting its mean and by dividing by its standard deviation, where the mean and the standard deviation are computed on the set of signal and background events used in the training of each classifier.

The boost and the rotation are used to remove the degeneracy among apparently different events which are instead equivalent under Lorentz transformation. "Feature scaling" is needed because the network works best with input variables of the same magnitude. The same preprocessing procedure, with the same mean and standard deviation values, is applied to all the events on which the classifiers are evaluated. After preprocessing, six of the twelve components of the light lepton, $\tau_{\text {had-vis }}$ and $E_{\mathrm{T}}^{\text {miss }}$ momenta are either vanishing or redundant, and therefore not included in the network inputs. The resulting lists of input variables are given in Table II. The transverse component, $p_{\mathrm{T}}^{\text {tot }}$, of the total momentum $p^{\text {tot }}$ in the lab frame is also included as otherwise this information would be lost after the preprocessing. The distributions of some of the NN input variables are shown in Sec. VII.

The NN classifiers are sequential models optimized for binary classification. They are based on the 
TABLE II. Input variables for the NN classifiers. The first six quantities are in the boosted and rotated frame described in the text; the last four are in the laboratory frame.

\begin{tabular}{llccc}
\hline \hline Variable & \multicolumn{1}{c}{ Description } & Z NN & Zll NN & W NN \\
\hline$\hat{E}^{\text {lep }}$ & light-lepton energy & $\checkmark$ & $\checkmark$ & $\checkmark$ \\
$\hat{p}_{x}^{\tau_{\text {had-vis }}}$ & $\tau_{\text {had-vis }} p_{x}$ & $\checkmark$ & $\checkmark$ & $\checkmark$ \\
$\hat{p}_{z}^{\tau_{\text {had-vis }}}$ & $\tau_{\text {had-vis }} p_{z}$ & $\checkmark$ & $\checkmark$ & $\checkmark$ \\
$\hat{E}_{\text {had-vis }}^{\tau_{\text {had-vis }}}$ & $\tau_{\text {hergy }}$ & $\checkmark$ & $\checkmark$ & $\checkmark$ \\
$\hat{p}_{z}^{\text {miss }}$ & $E_{\mathrm{T}}^{\text {miss }}$ component along & $\checkmark$ & $\checkmark$ & $\checkmark$ \\
$\hat{E}^{\text {miss }}$ & $\quad z$ axis \\
$p_{\mathrm{T}}^{\text {tot }}$ & magnitude of $E_{\mathrm{T}}^{\text {miss }}$ & $\checkmark$ & $\checkmark$ & $\checkmark$ \\
& transverse component & $\checkmark$ & $\checkmark$ & $\checkmark$ \\
$m_{\text {coll }}$ & $\quad$ of total momentum & & & \\
$\Delta \alpha$ & collinear mass & $\checkmark$ & $\checkmark$ & $\checkmark$ \\
$m\left(\ell, \tau_{\text {had-vis }}\right)$ & invariant mass of light & & $\checkmark$ & $\checkmark$ \\
& lepton and $\tau_{\text {had-vis }}$ & & & \\
\hline \hline
\end{tabular}

KERAS 1.1.1 [49] and TENSORFLOW 0.11 [50] packages, using a standard implementation for binary classifiers having two hidden dense layers with 16 nodes each.

In order to obtain a single discriminating variable, the outputs of the classifiers evaluated in each event are combined in the following way. In events with 3-prong $\tau_{\text {had-vis }}$ candidates, where no further rejection is needed against the $Z \rightarrow \ell \ell$ events, the $\mathrm{Z}$ and $\mathrm{W}$ classifiers are combined as the distance in the two-dimensional plane from the point with highest $\mathrm{NN}$ outputs, where the $\mathrm{NN}$ outputs can range within $[0,1]$ :

combined output (3P)

$$
=1-\sqrt{\left(1-\text { output }_{\mathrm{W}}\right)^{2}+\left(1-\text { output }_{\mathrm{Z}}\right)^{2}} / \sqrt{2} .
$$

In a similar fashion, for events with 1-prong $\tau_{\text {had-vis }}$ candidates, the Z, W and Zll classifiers are combined as

$$
\text { combined output }(1 \mathrm{P})=1-\sqrt{\left(1-\text { output }_{\mathrm{W}}\right)^{2}+\left(1-\text { output }_{\mathrm{Z}}\right)^{2}+\left(1-\text { output }_{\mathrm{ZII}}\right)^{2}} / \sqrt{3} \text {. }
$$

The chosen procedure to combine the individual NN outputs reduces the dimensionality of the classifiers while maintaining the correlations among these classifiers for each event. The binned distributions of these combined classifiers for the events selected in the SR are used in the final template fit, as discussed in Sec. VII.

\section{BACKGROUND ESTIMATION}

Background processes are categorized according to the origin of the $\tau_{\text {had-vis }}$ candidate, which can be a true $\tau$ lepton, or a misidentified light lepton, or a misidentified quark- or gluon-initiated jet. Different techniques are used to estimate these background contributions in the SR, as well as to model their expected combined $\mathrm{NN}$ output distributions, which are used in the template fit to data (Sec. VII). As described in the following, the shapes of all components are determined prior to the fit, as are the normalizations for all but the $Z \rightarrow \tau \tau$ and fake components, which are determined in the fit.

Backgrounds from processes with a true hadronically decaying $\tau$ lepton are estimated from simulation. The $Z \rightarrow$ $\tau \tau$ decays are the dominant source of these events. As detailed in Sec. III, they are modeled via simulation but their total yield in the SR is left unconstrained in the template fit to data in order to remove the theoretical systematic uncertainties in the $Z$ production cross section.

Processes where the $\tau_{\text {had-vis }}$ candidate is a misidentified light lepton are also estimated from simulation. These are mostly $Z \rightarrow \ell \ell$ events. The simulated rate for misidentifying electrons as 1-prong $\tau_{\text {had-vis }}$ candidates is corrected using data [24]. Due to the lack of dedicated measurements of the rates of misidentifying electrons as 3 -prong $\tau_{\text {had-vis }}$ candidates and muons as 1-prong $\tau_{\text {had-vis }}$ candidates, conservative uncertainties are assigned which have negligible impact on the precision of the measured $\mathcal{B}(Z \rightarrow \ell \tau)$.

The normalization of the $Z \rightarrow \ell \ell$ events is determined from data with a sample of events with an opposite-charge muon pair with $81 \mathrm{GeV}<m_{\mu \mu}<101 \mathrm{GeV}$. The preselection requirements on the leading muon, the absence of $b$-tagged jets and the veto on additional light leptons are imposed. A correction factor derived as the relative difference between the predicted and observed numbers of $Z \rightarrow \mu \mu$ events is applied to both the $Z \rightarrow e e$ and $Z \rightarrow \mu \mu$ yields in the SR. This correction is applied as a function of the reconstructed transverse momentum of the $Z / \gamma^{*}$ boson to correct the overall normalization as well as the $p_{\mathrm{T}}\left(Z / \gamma^{*}\right)$ distribution of the simulated $Z \rightarrow \ell \ell$ events. In the $Z \rightarrow \mu \mu$-enhanced region, the $Z / \gamma^{*}$ boson momentum is computed as the vector sum of the muon pair, while in the SR it is the vector sum of the misidentified $\tau_{\text {had-vis }}$ candidate and the remaining light lepton. The uncertainty in this correction is statistical only. Differences between the electron and muon acceptances are covered by the systematic uncertainties in the electron and muon selections, which are accounted for in the $Z \rightarrow \ell \ell$ predictions in the SR.

Events where the $\tau_{\text {had-vis }}$ candidate originates from a quark- or gluon-initiated jet are estimated from data. This contribution is referred to as "fakes" and is dominated by $W+$ jets and multijet processes. A data-driven fake-factor technique is used to estimate this contribution. It uses events in the so-called "fail sideband," which is the set of events passing all but one of the SR selection requirements: 
TABLE III. Calibration regions used to derive fake factors. Differences from the SR selection (Table I) are listed together with the purities for the target processes as expected from simulation.

\begin{tabular}{llll}
\hline \hline & & \multicolumn{2}{c}{ Purity [\%] } \\
\cline { 3 - 4 } Region & $\quad$ Change relative to SR selection & $e \tau$ & $\mu \tau$ \\
\hline CRZll & Two same-flavor opposite-charge light & 98 & 98 \\
& $\quad \begin{array}{l}\text { leptons with } 81<m_{\ell \ell}<101 \mathrm{GeV} \\
\text { CRW }\end{array}$ & & \\
& $m_{\mathrm{T}}\left(\ell, E_{\mathrm{T}}^{\text {miss }}\right)>40 \mathrm{GeV}$ and \\
& $m_{\mathrm{T}}\left(\tau_{\text {had-vis }}, E_{\mathrm{T}}^{\text {miss }}\right)>35(30) \mathrm{GeV}$ & & 85 \\
in $e \tau(\mu \tau)$ events & & \\
CRT & $N_{b \text {-jets }} \geq 2$ & 98 & 98 \\
CRQ & Inverted light-lepton isolation & 75 & 37 \\
\hline \hline
\end{tabular}

the $\tau_{\text {had-vis }}$ candidate is required to fail the tight identification requirement. This is a set of events orthogonal to the ones selected in the SR and enhanced with fakes. The yield of these events is corrected by the fake factor, which is the transfer factor needed to scale the fail sideband sample to the amount of background expected in the signal region, which requires an identified $\tau_{\text {had-vis }}$ candidate. This factor is process-specific as it depends on the fractions of quark- and gluon-initiated jets that are misidentified as $\tau_{\text {had-vis }}$ candidates. It also depends on properties of the $\tau_{\text {had-vis }}$ candidate. To capture these effects, different fake factors are measured in samples of events dominated by different processes and different $\tau_{\text {had-vis }}$ kinematic properties.

Fake factors $F_{\mathrm{W}}, F_{\mathrm{T}}, F_{\mathrm{Zll}}$, and $F_{\mathrm{QCD}}$ are measured in four data samples of events dominated by $W+$ jets ("CRW"), $t \bar{t}$ and single-top ("CRT"), $Z \rightarrow \ell \ell+$ jets ("CRZIl"), and multijet ("CRQ") events, respectively. The selections that define these "calibration regions" (CR) are similar to the SR selection but define orthogonal samples dominated by the target source of background. These selections are detailed in Table III together with the expected purities in each $\mathrm{CR}$ for the target process as estimated from simulation. For CRQ the purity is estimated as the number of events in data, after subtracting the contribution from other processes estimated from simulation, divided by the total number of events.

In each CR, $F_{i}(i=\mathrm{W}, \mathrm{T}, \mathrm{Zll}, \mathrm{QCD})$ is measured in data as the ratio of the number of events where the $\tau_{\text {had-vis }}$ candidate passes the tight identification to the number of events where the $\tau_{\text {had-vis }}$ candidate fails in bins of the $\tau_{\text {had-vis }}$ $p_{\mathrm{T}}$. Contributions from background processes that are not the target process of the CR or from events where the $\tau_{\text {had-vis }}$ candidate does not originate from a jet are subtracted from data using simulation. The four $F_{i}$ are combined into a weighted average $F=\sum_{i} R_{i} F_{i}$, where $R_{i}$ is the fraction of events from fakes in the SR as predicted by simulation for each process. For multijet events, this fraction is defined as $R_{\mathrm{QCD}}=1-R_{\mathrm{W}}-R_{\mathrm{Zll}}-R_{\mathrm{T}}$. Fake factors are measured separately for $\tau_{\text {had-vis }}$ candidates with one and with three associated tracks. For 1-prong candidates, they are estimated in two-dimensional bins of $\tau_{\text {had-vis }} p_{\mathrm{T}}$ and $\tau_{\text {had-vis }}$ track $p_{\mathrm{T}}$, since the associated track momentum is used in the selection of these candidates, while for 3-prong candidates they are estimated only in bins of $\tau_{\text {had-vis }} p_{\mathrm{T}}$. The choice of bin boundaries is optimized to capture the statistically significant variations of the fake factors as a function of the $\tau_{\text {had-vis }}$ properties, while retaining enough events per bin. An additional binning as a function of $\tau_{\text {had-vis }}|\eta|$ was found to be unnecessary. The measured fake factors are shown in Table IV. For events with low $\tau_{\text {had-vis }}$ $p_{\mathrm{T}}$ and high $\tau_{\text {had-vis }}$ track $p_{\mathrm{T}}$, the fake factors are large and have large statistical uncertainties because there are few events in the calibration regions. However, these fake factors are applied only to a small fraction of events in the sidebands.

The number of events from fakes in the SR is

$$
N_{\mathrm{SR}}^{\mathrm{fake}}=\sum_{k}\left(N_{\mathrm{SR}, \mathrm{data}}^{\mathrm{fail}}-N_{\mathrm{SR}, \mathrm{MC}, \text { not jet } \rightarrow \tau}^{\mathrm{fail}}\right)_{k} \times F_{k},
$$

where $F_{k}$ is the fake factor corresponding to the $p_{\mathrm{T}}$ (and track $p_{\mathrm{T}}$ for 1-prong $\tau_{\text {had-vis }}$ ) bin $k, N_{\mathrm{SR} \text {,data }}^{\text {fail }}$ is the number of

TABLE IV. The fake factors binned in $\tau_{\text {had-vis }} p_{\mathrm{T}}$ and $\tau_{\text {had-vis }}$ track $p_{\mathrm{T}}$ for 1-prong, and $\tau_{\text {had-vis }} p_{\mathrm{T}}$ for 3-prong events as determined in the SR.

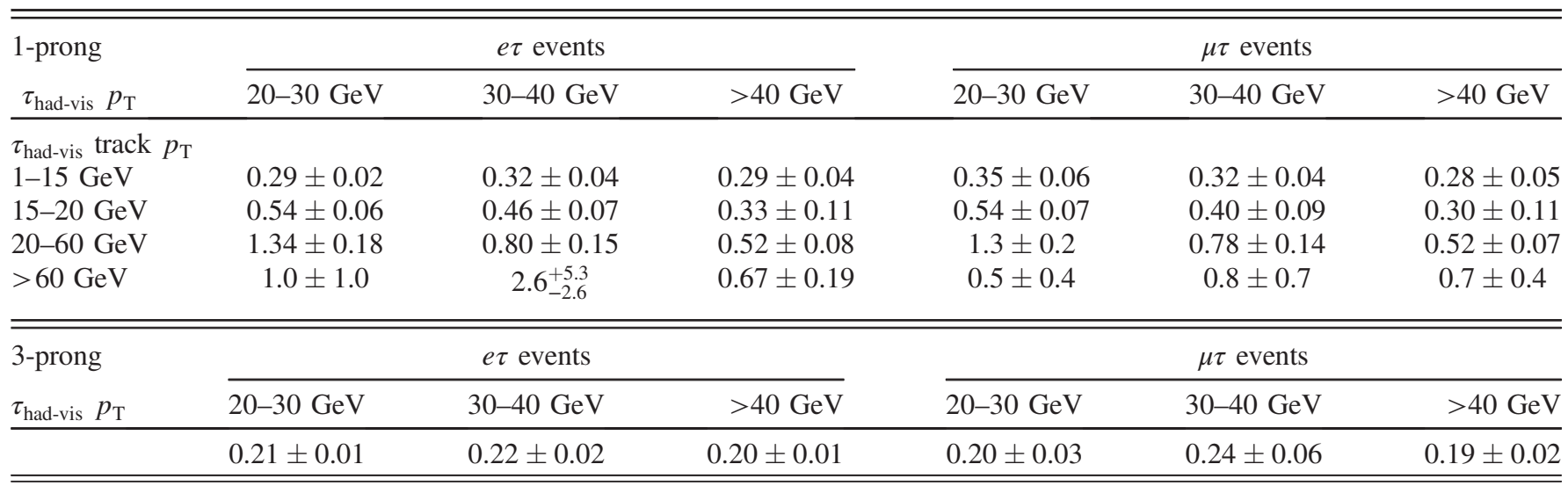


data events in the fail sideband in bin $k$, and $N_{\mathrm{SR}, \mathrm{MC} \text {,not jet } \rightarrow \tau}^{\mathrm{fail}}$ is the number of events in the fail sideband in bin $k$ for which the $\tau_{\text {had-vis }}$ candidate did not originate from a jet as predicted by simulation.

The sources of uncertainty in the estimate of the fake background are the statistical uncertainties in the $F$ measurements in each bin, the statistical uncertainties of the data in the fail sideband and the uncertainty in $R_{i}$. All statistical uncertainties are treated as independent. The uncertainty in $R_{i}$ is estimated by varying the estimated $R_{\mathrm{W}}$ by $50 \%$, although this has a negligible impact on the sensitivity.

The simulation and the data-driven techniques used to model the signal and background processes were validated in samples enriched with fakes and $Z \rightarrow \tau \tau$ events. Both the predicted NN input and output distributions are in agreement with data.

\section{SYSTEMATIC UNCERTAINTIES}

Systematic uncertainties affecting the estimations of signal and background contributions arise from the
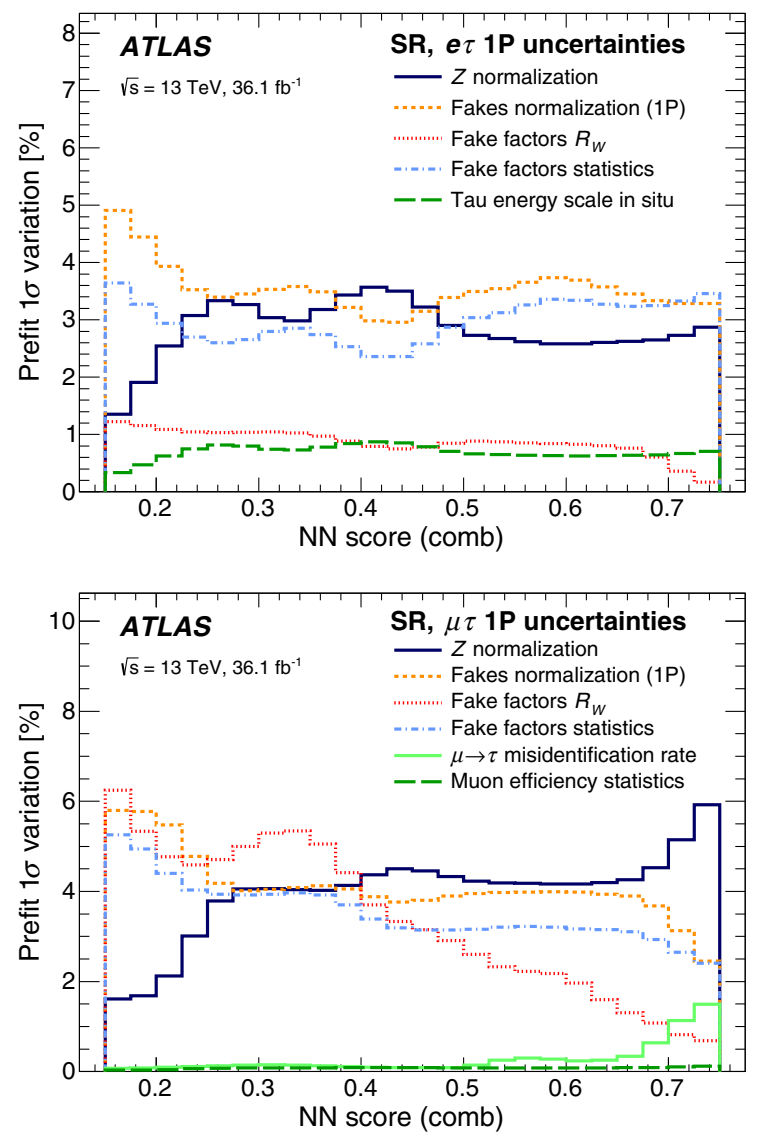

theoretical predictions and the detector modeling used in simulation, the luminosity measurement, and the datadriven background estimations.

The theoretical uncertainties in the production cross section affect only the predictions of the simulated $W+$ jets, top, diboson and Higgs boson events with a true hadronically decaying $\tau$ lepton, since the $Z \rightarrow \tau \tau$ and signal yields are determined in the template fit to data. These constitute a small fraction of the background events in the $\mathrm{SR}$, and a conservative uncertainty in their production cross sections was assigned with negligible impact on the final results. As described in Sec. V, $Z \rightarrow \ell \ell$ events are normalized to data using $Z \rightarrow \mu \mu$ events, so the theoretical uncertainty in the $Z \rightarrow \ell \ell$ normalization is irrelevant. The statistical uncertainty of $0.1 \%$ in this normalization correction is included as a systematic uncertainty.

Uncertainties arising from the simulation of the detector and pileup conditions in the reconstruction of $\tau_{\text {had-vis }}$ candidates, muons, electrons, jets (including $b$-tagging) and $E_{\mathrm{T}}^{\text {miss }}$ are evaluated. Sources of uncertainty in the $\tau_{\text {had-vis }}$ candidate include the reconstruction and identification
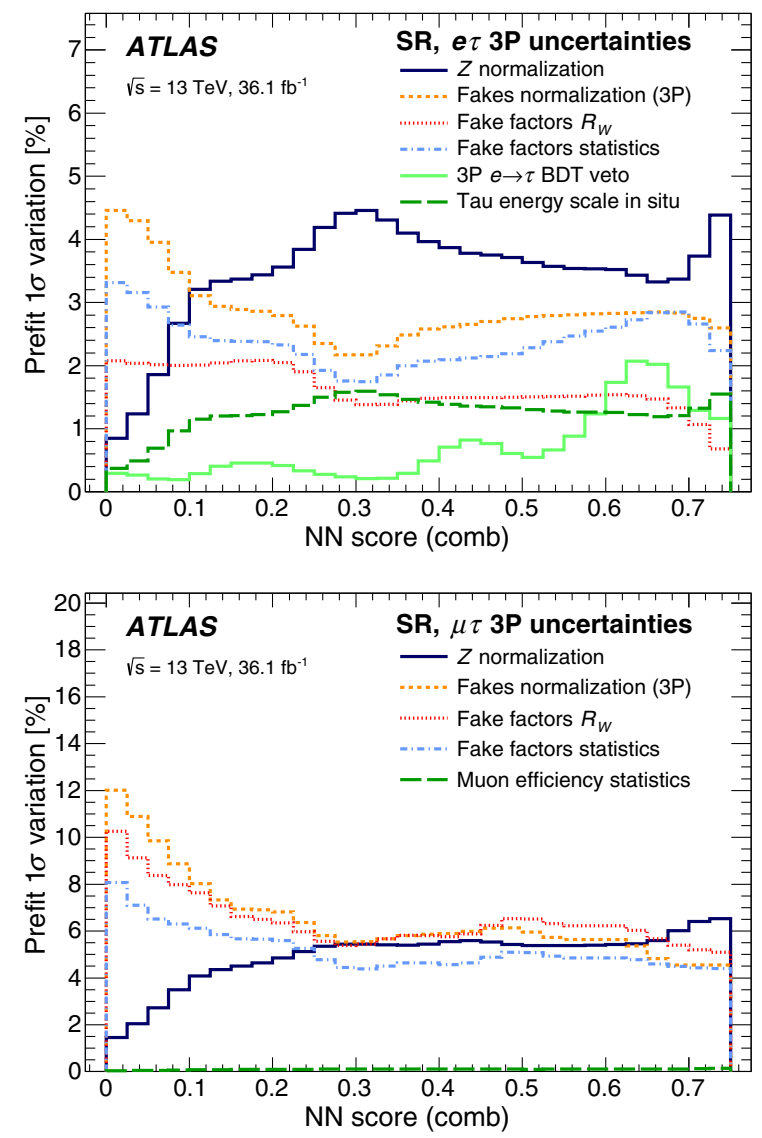

FIG. 3. Expected uncertainties in the total background predictions in the SR as a function of the combined NN output for the dominant systematic uncertainties in $e \tau$ (top) and $\mu \tau$ (bottom) channels with 1-prong (left) and 3-prong (right) $\tau_{\text {had-vis }}$ candidates. The uncertainties in the normalizations of the $Z$ and fake components are based on the expected statistical power of the fit described in Sec. VII. "Muon efficiency statistics" refers to the statistical uncertainty of the corrections applied to the simulated muon reconstruction efficiency [14]. "Tau energy scale in situ" refers to the uncertainty of the corrections applied to the energy of the $\tau_{\text {had-vis }}$ candidate based on measurements with $Z \rightarrow \tau \tau$ data [24]. 
efficiencies and the energy calibration. These are applied only to $\tau_{\text {had-vis }}$ candidates from hadronically decaying $\tau$ leptons. For misidentified $\tau_{\text {had-vis }}$ candidates originating from an electron or a muon, systematic uncertainties in the misidentification rates are assigned using a data-driven method, as detailed in Sec. V. For the simulation of electron and muon candidates, uncertainties in the trigger, reconstruction, identification and isolation efficiencies are accounted for. The effect of uncertainties in the light-lepton momentum scale and resolution is also evaluated. For jets, uncertainties in the jet momentum scale and resolution, as well as in the $b$-tagging (in)efficiencies are accounted for. All experimental uncertainties are propagated to the $E_{\mathrm{T}}^{\text {miss }}$ calculation. In addition, uncertainties in the energy scale and resolution of the $E_{\mathrm{T}}^{\text {miss }}$ soft term are considered.

The $2.1 \%$ uncertainty in the measured luminosity (Sec. III) is only considered for the simulated $W+$ jets, top, diboson and Higgs boson contributions, whose normalizations are based purely on simulation, without any data-driven estimate.

Data-driven techniques are used to estimate the background contributions from events with a $\tau_{\text {had-vis }}$ candidate originating from either a light lepton or a quark- or gluoninitiated jet. The systematic uncertainties in these methods are described in Sec. V.

To illustrate the sizes of the systematic uncertainties, Fig. 3 shows the relative uncertainties of the total background predictions as a function of the combined NN output for the dominant systematic uncertainties. The uncertainties in the normalizations of the $Z$ and fake components, estimated from the expected statistical power of the fit described in Sec. VII, and the statistical uncertainty in the fake factor are the largest sources of systematic uncertainty, contributing on average between $3 \%$ and $6 \%$. The systematic uncertainty in $R_{\mathrm{W}}$ is also relevant and ranges between $1 \%$ and $6 \%$ over the different final states. All other systematic uncertainties affect the total background prediction by less than one percent.

\section{RESULTS AND STATISTICAL INTERPRETATION}

A binned maximum-likelihood fit to data, performed with the statistical analysis packages RooFIT [51], RooStats [52] and HistFitTER [53], is used to compare the observed binned distributions of the combined NN classifiers in the SR with the model, and to extract evidence of signal events. The parameter of interest in such fit is the signal strength modifier $\mu_{\text {sig, }}$, which quantifies the size of the LFV decay branching fraction $\mathcal{B}(Z \rightarrow \ell \tau)$.

Fits are performed independently for the $e \tau$ and $\mu \tau$ channels, and in each fit events with a 1-prong $\tau_{\text {had-vis }}$ candidate and those with a 3-prong candidate are considered separately. In the fits of events with 1-prong $\tau_{\text {had-vis }}$ candidates, because of the way the NN classifiers are combined, only a few background-like events have an NN output value below 0.15; these are excluded. Independent templates, estimated as described in previous sections, are used for signal, $Z \rightarrow \tau \tau$, fakes, $Z \rightarrow \ell \ell$, top events, and $W(\rightarrow \tau \nu)+$ jets events. The small contributions from Higgs boson and diboson events are summed into a single template, referred to as "Other."

The likelihood is the product of Poisson probability density functions describing the observed number of events in each bin. It also includes Gaussian, Poisson and lognormal distributions to constrain the nuisance parameters associated with the systematic, statistical and theoretical uncertainties in the predicted number of events, respectively. In addition to the parameter of interest and the nuisance parameters, three normalization parameters are included: $\mu(Z)$ determines the normalizations of the $Z \rightarrow \tau \tau$ and signal events while $\mu$ (fakes $1 \mathrm{P})$ and $\mu$ (fakes3P) control the normalization of the fake component in events with a 1-prong or a 3 -prong $\tau_{\text {had-vis }}$ candidate, respectively. These parameters are fit independently in the $e \tau$ and $\mu \tau$ channels. Within the same channel, the same $\mu(Z)$ is used to fit events with 1-prong and 3-prong $\tau_{\text {had-vis }}$ candidates, while $\mu$ (fakes $1 \mathrm{P})$ and $\mu$ (fakes $3 \mathrm{P})$ are used to fit independently the corresponding contributions from fakes. By fitting the overall normalizations of the $Z \rightarrow \tau \tau$ and signal event yields, the $\mu(Z)$ parameter accounts for uncertainties in these contributions due to theoretical uncertainties on the $Z$ production cross section

TABLE V. The total observed number of events and postfit event yields in the SR for the $e \tau$ (top) and $\mu \tau$ (bottom) channels after a fit to data. The uncertainties include both the statistical and systematic contributions. The correlations between the uncertainties in individual contributions are accounted for in the quoted uncertainties in the total postfit event yields.

\begin{tabular}{lcc}
\hline \hline & 1-prong & 3-prong \\
\hline Total observed $e \tau$ events & 89294 & 35148 \\
Total postfit $e \tau$ event yield & $89300 \pm 300$ & $35200 \pm 200$ \\
Fakes & $57000 \pm 1000$ & $21500 \pm 500$ \\
$Z \rightarrow \tau \tau$ & $26000 \pm 1000$ & $11500 \pm 500$ \\
$Z \rightarrow \ell \ell$ & $3200 \pm 100$ & $250 \pm 150$ \\
Top & $770 \pm 120$ & $440 \pm 70$ \\
$W+$ jets & $540 \pm 100$ & $950 \pm 180$ \\
Other & $340 \pm 70$ & $150 \pm 30$ \\
$Z \rightarrow e \tau$ signal & $900 \pm 400$ & $390 \pm 160$ \\
\hline Total observed $\mu \tau$ events & 79744 & 25050 \\
Total postfit $\mu \tau$ event yield & $79700 \pm 500$ & $25100 \pm 700$ \\
Fakes & $52000 \pm 1000$ & $13600 \pm 800$ \\
$Z \rightarrow \tau \tau$ & $26000 \pm 1000$ & $10300 \pm 300$ \\
$Z \rightarrow \ell \ell$ & $240 \pm 110$ & $80 \pm 40$ \\
Top & $890 \pm 140$ & $360 \pm 60$ \\
$W+$ jets & $610 \pm 120$ & $680 \pm 130$ \\
Other & $290 \pm 70$ & $110 \pm 20$ \\
$Z \rightarrow \mu \tau$ signal & $-20 \pm 360$ & $-10 \pm 140$ \\
\hline \hline
\end{tabular}


as well as experimental uncertainties in the measurement of the integrated luminosity and in the acceptance times efficiency of the $\ell \tau$ final state (uncertainties due to trigger, reconstruction, isolation and identification efficiencies). Therefore, $\mu(Z)$ ensures that the same $Z$ production cross section and the same $\ell \tau$ acceptance efficiencies are used in the predictions of the signal and the $Z \rightarrow \tau \tau$ background contribution. The normalization with $\mu(Z)=1$ corresponds to the $Z$ production cross section of $2.1 \mathrm{nb}$, the SM value calculated at NNLO accuracy, multiplied by the nominal detector acceptances and the measured integrated luminosity. The normalization parameters $\mu$ (fakes $1 \mathrm{P})$ and $\mu$ (fakes $3 \mathrm{P})$ account for the systematic uncertainties in the overall normalizations of the fake contributions, so that
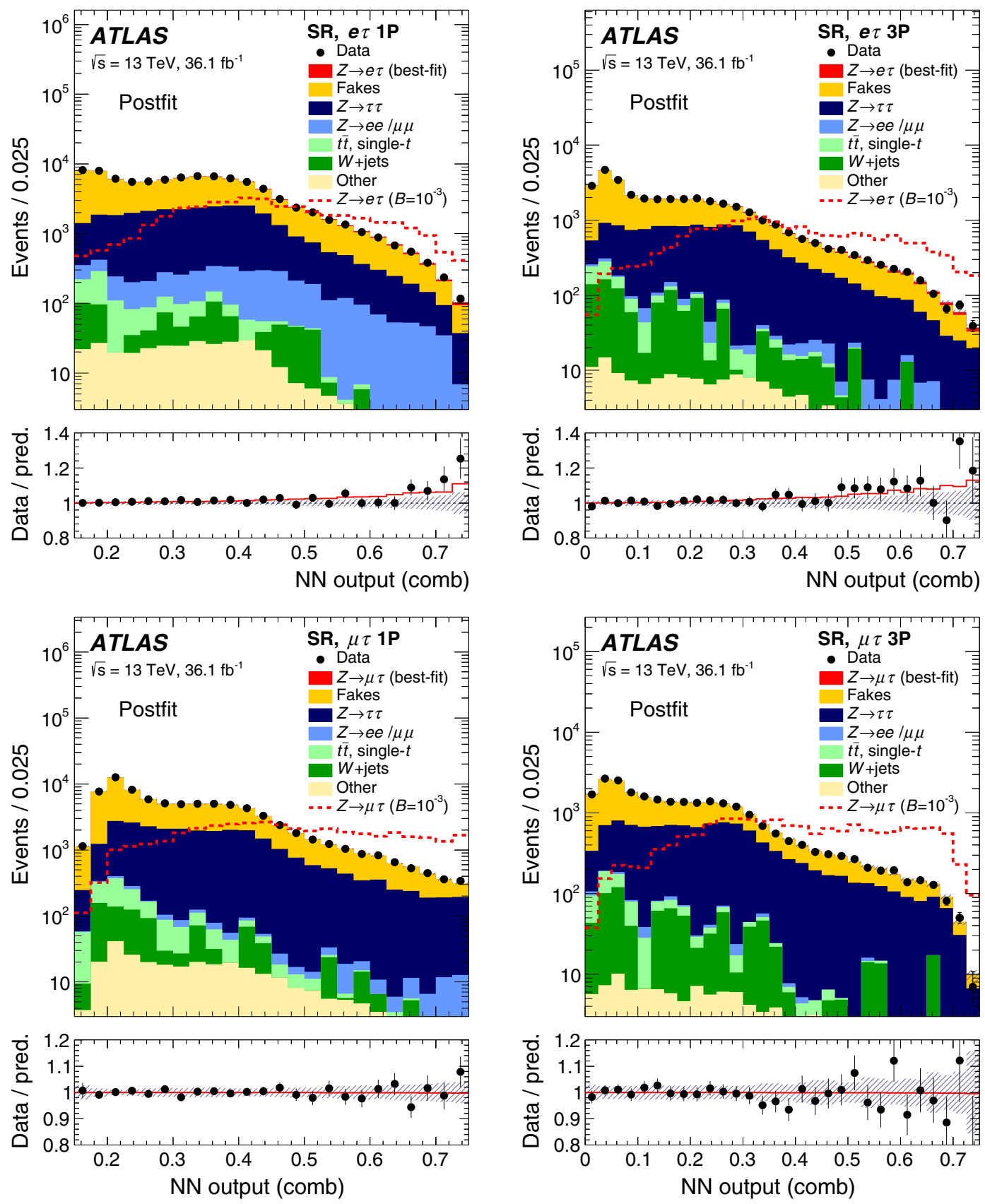

FIG. 4. Observed and expected postfit distributions of the combined NN output in SR for the $e \tau$ (top) and $\mu \tau$ (bottom) channels, for 1-prong (left) and 3-prong (right) $\tau_{\text {had-vis }}$ candidates. The filled histogram stacked on top of the backgrounds represents the signal normalized to the best-fit $\mathcal{B}(Z \rightarrow \ell \tau)$. The overlaid dashed line represents the expected distribution for the signal normalized to $\mathcal{B}(Z \rightarrow \ell \tau)=10^{-3}$. In the panels below each plot, the ratios of the observed data (dots) and the postfit background plus signal (solid line) to the postfit background are shown. The hatched error bands represent the combined statistical and systematic uncertainties. The first and last bins include underflow and overflow events, respectively. 
TABLE VI. Best-fit values for $\mathcal{B}(Z \rightarrow \ell \tau)$ and the other free parameters, and exclusion upper limits in the $e \tau$ and $\mu \tau$ channels. The uncertainties include both the statistical and systematic contributions.

\begin{tabular}{lcc}
\hline \hline & $e \tau$ & $\mu \tau$ \\
\hline $\mathcal{B}(Z \rightarrow \ell \tau)$ & $\left(3.3_{-1.4}^{+1.5}\right) \times 10^{-5}$ & $\left(-0.1_{-1.2}^{+1.2}\right) \times 10^{-5}$ \\
$\mu(Z)$ & $0.83_{-0.07}^{+0.09}$ & $0.87_{-0.08}^{+0.09}$ \\
$\mu($ fakes 1P) & $1.18_{-0.06}^{+0.06}$ & $1.12_{-0.08}^{+0.09}$ \\
$\mu($ fakes3P) & $1.01_{-0.05}^{+0.06}$ & $1.09_{-0.14}^{+0.13}$ \\
Observed (expected) upper & $5.8(2.8) \times 10^{-5}$ & $2.4(2.4) \times 10^{-5}$ \\
$\quad$ limit at 95\% C.L. & & \\
\hline \hline
\end{tabular}

only the systematic uncertainties in the template shape are implemented as nuisance parameters. The fitted values of these parameters are sensitive to the yields of events with low NN outputs, which are dominated by contributions from $Z \rightarrow \tau \tau$ and fakes. Fitting these normalization parameters reduces the systematic uncertainties in the predictions of the $Z \rightarrow \tau \tau$ and fake backgrounds in the bins at high NN output, which are sensitive to the $Z \rightarrow \ell \tau$ signal.

Table $\mathrm{V}$ reports the total observed number of events and postfit event yields in the SR after a fit to data. The observed and postfit expected distributions of the combined NN output are shown in Fig. 4. As reported in Table VI, the best-fit values for $\mu(Z), \mu($ fakes $1 \mathrm{P})$ and $\mu($ fakes $3 \mathrm{P})$ are consistent between the $e \tau$ and $\mu \tau$ channels, while the bestfit value for $\mathcal{B}(Z \rightarrow \ell \tau)$ is consistent with zero in the $\mu \tau$

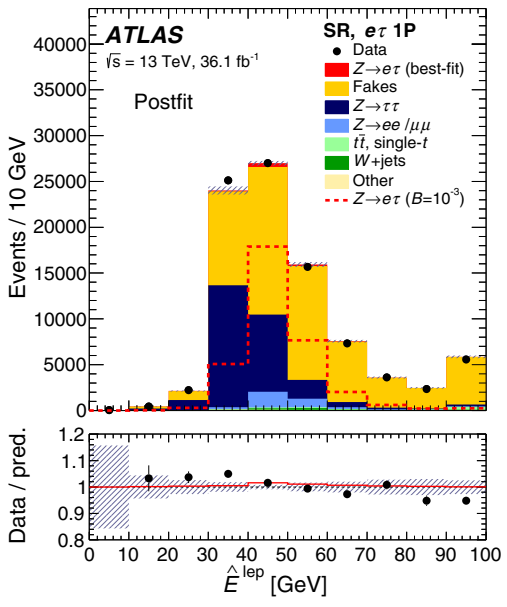

(a) $\hat{E}^{\text {lep }}$

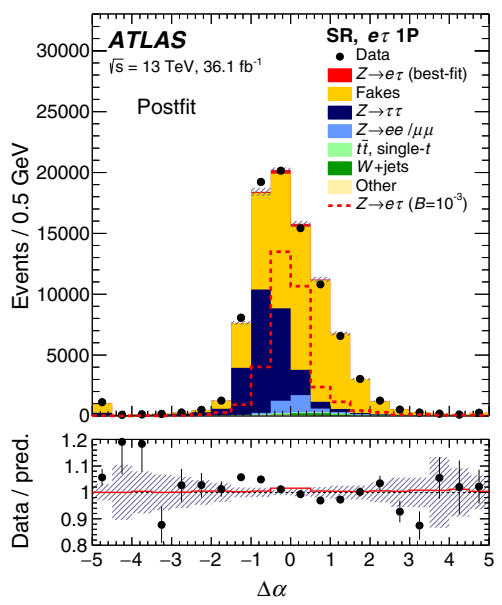

(d) $\Delta \alpha$

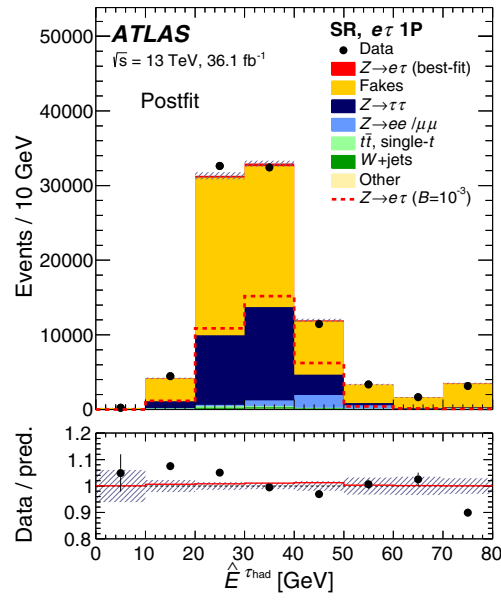

(b) $\hat{E}^{\tau_{\text {had }}}$

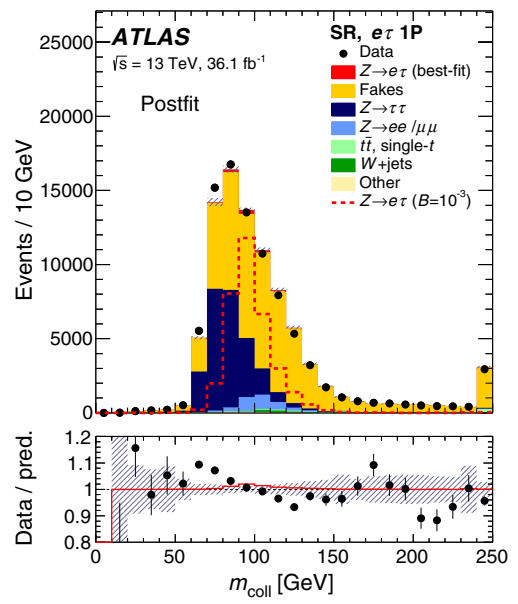

(e) $m_{\text {coll }}$

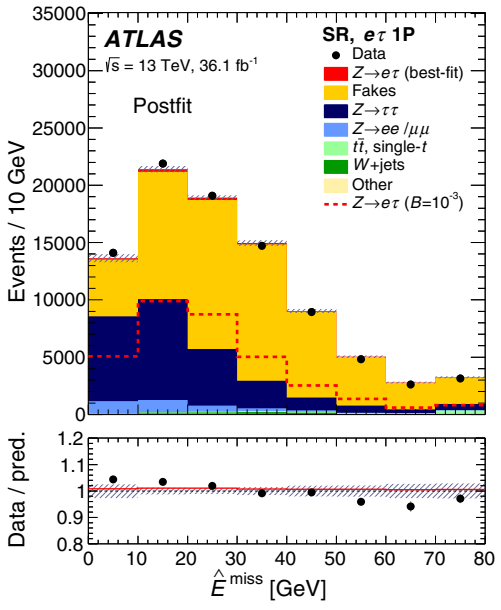

(c) $\hat{E}^{\text {miss }}$

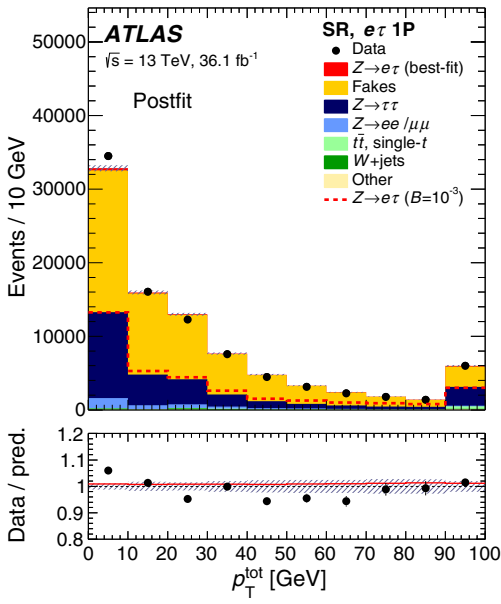

(f) $p_{\mathrm{T}}^{\text {tot }}$

FIG. 5. Observed and expected postfit distributions of unscaled NN inputs in SR for the $e \tau$ channel with 1-prong $\tau_{\text {had-vis }}$ candidates. The fit is based on profiling on the combined NN classifier, but not directly on these variables. The filled histogram stacked on top of the backgrounds represents the signal normalized to the best-fit $\mathcal{B}(Z \rightarrow \ell \tau)$. The overlaid dashed line represents the expected distribution for the signal normalized to $\mathcal{B}(Z \rightarrow \ell \tau)=10^{-3}$. In the panels below each plot, the ratios of the observed data (dots) and the postfit background plus signal (solid line) to the postfit background are shown. The hatched error bands represent the combined statistical and systematic uncertainties. The first and last bins include underflow and overflow events, respectively. 
channel, $\mathcal{B}(Z \rightarrow \mu \tau)=\left(-0.1_{-1.2}^{+1.2}\right) \times 10^{-5}$, and slightly deviates from zero in the $e \tau$ channel, $\mathcal{B}(Z \rightarrow e \tau)=\left(3.3_{-1.4}^{+1.5}\right) \times 10^{-5}$.

Observed and expected postfit distributions of the unscaled NN inputs of the events in the SR are shown in Figs. 5-8. The postfit distributions are compatible with data. An alternative fit combining the $e \tau$ and $\mu \tau$ channels with two independent parameters of interest and the same shared free parameter $\mu(Z)$ yielded the same results for the signal branching fractions and for the background normalization parameters as in the nominal fit. The compatibility of the normalizations of the $Z \rightarrow \tau \tau$ background in events with 1-prong or 3-prong $\tau_{\text {had-vis }}$ candidates was also tested by fitting these two contributions independently. The best-fit values for all the normalization parameters were compatible in less than $1 \sigma$ with the values obtained in the nominal fit and no significant differences were observed in the upper limits on the signal branching ratios.

After the fit, the probabilities of compatibility between the data and the background-plus-signal and backgroundonly hypotheses are assessed using the profile loglikelihood ratio method [54], where the nuisance parameters are profiled as a function of the parameter of interest. The normalization parameters are not profiled. As no significant deviation from the background-only hypothesis is observed, exclusion upper limits on $\mathcal{B}(Z \rightarrow \ell \tau)$ are set using the $\mathrm{CL}_{\mathrm{s}}$ method [55]. The resulting observed (expected) upper limits at $95 \%$ C.L. are $\mathcal{B}(Z \rightarrow e \tau)<$ $5.8 \times 10^{-5}\left(2.8 \times 10^{-5}\right)$ and $\mathcal{B}(Z \rightarrow \mu \tau)<2.4 \times 10^{-5}$ $\left(2.4 \times 10^{-5}\right)$. The significance of the excess in the $e \tau$ channel is $2.3 \sigma$.

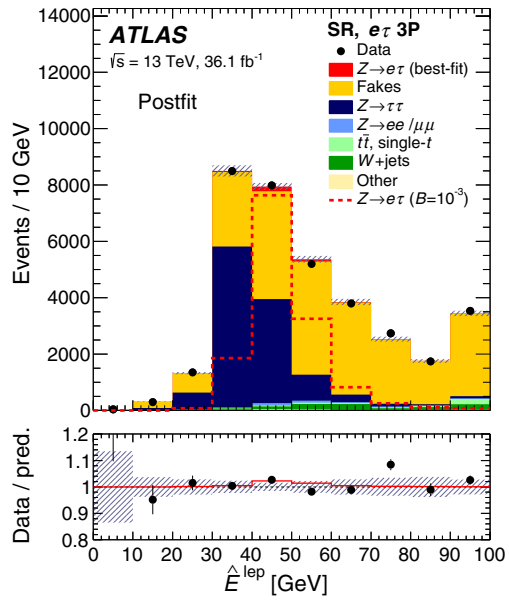

(a) $\hat{E}^{\mathrm{lep}}$

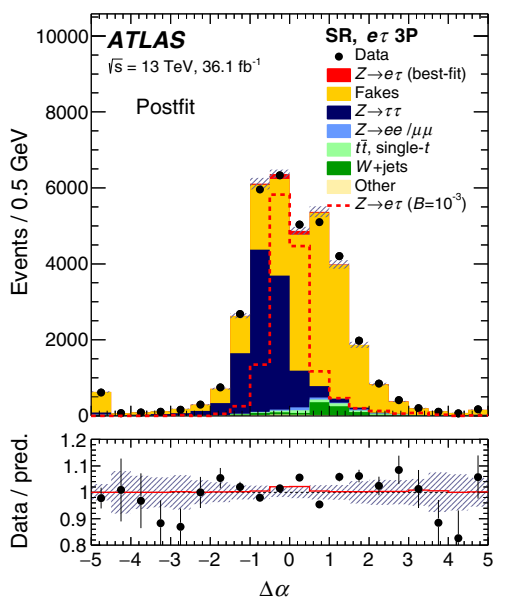

(d) $\Delta \alpha$

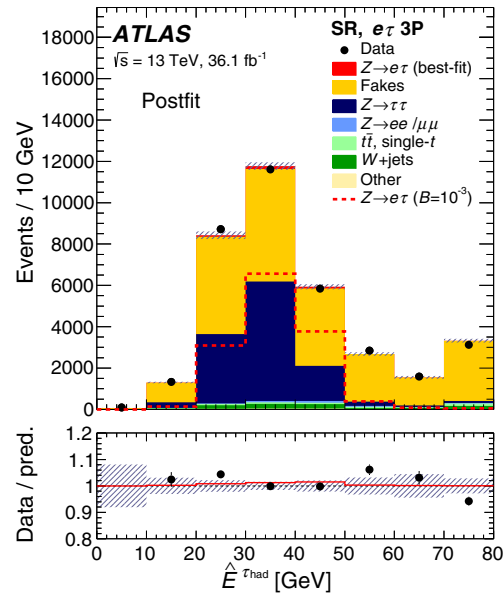

(b) $\hat{E}^{\tau_{\text {had }}}$

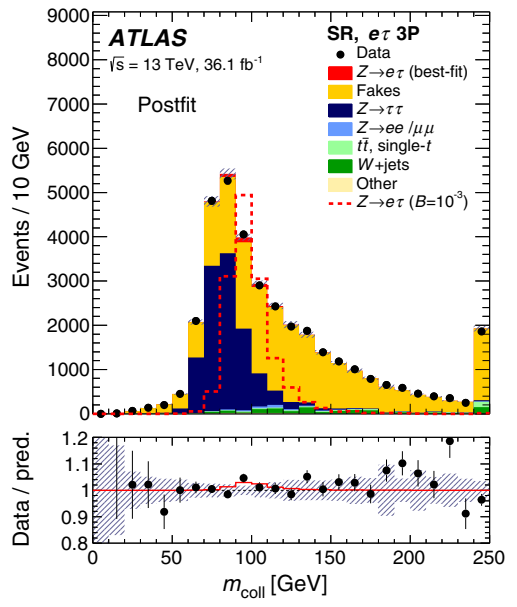

(e) $m_{\text {coll }}$

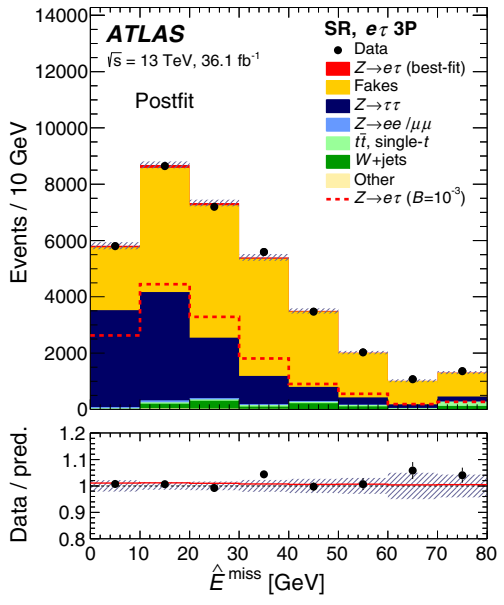

(c) $\hat{E}^{\text {miss }}$

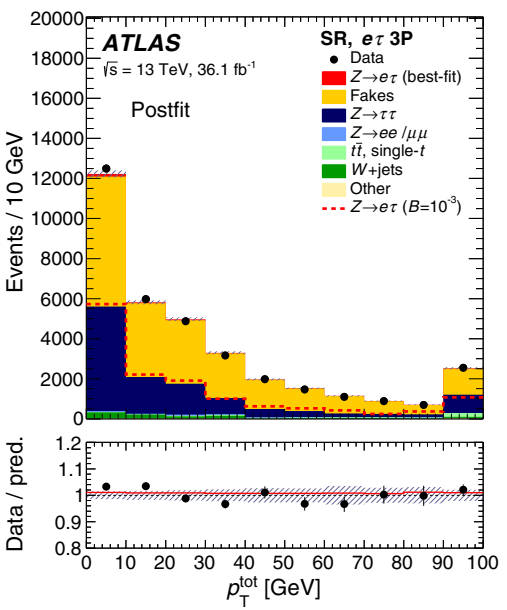

(f) $p_{\mathrm{T}}^{\text {tot }}$

FIG. 6. Observed and expected postfit distributions of unscaled NN inputs in SR for the $e \tau$ channel with 3-prong $\tau_{\text {had-vis }}$ candidates. The fit is based on profiling on the combined NN classifier, but not directly on these variables. The filled histogram stacked on top of the backgrounds represents the signal normalized to the best-fit $\mathcal{B}(Z \rightarrow \ell \tau)$. The overlaid dashed line represents the expected distribution for the signal normalized to $\mathcal{B}(Z \rightarrow \ell \tau)=10^{-3}$. In the panels below each plot, the ratios of the observed data (dots) and the postfit background plus signal (solid line) to the postfit background are shown. The hatched error bands represent the combined statistical and systematic uncertainties. The first and last bins include underflow and overflow events, respectively. 


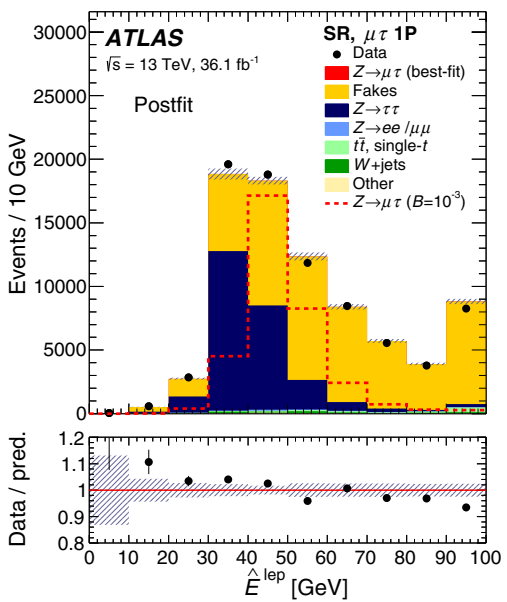

(a) $\hat{E}^{\text {lep }}$

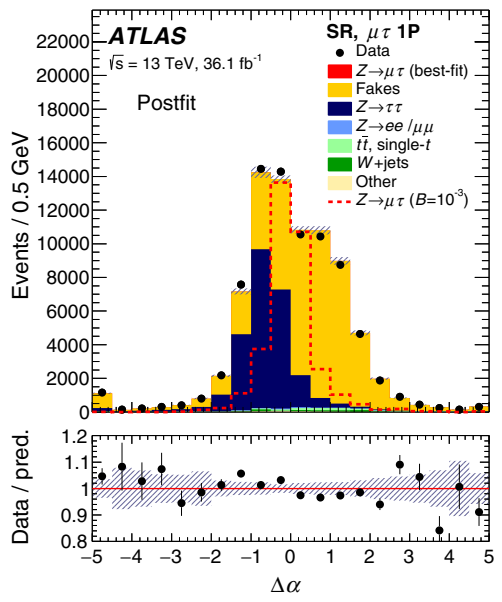

(d) $\Delta \alpha$

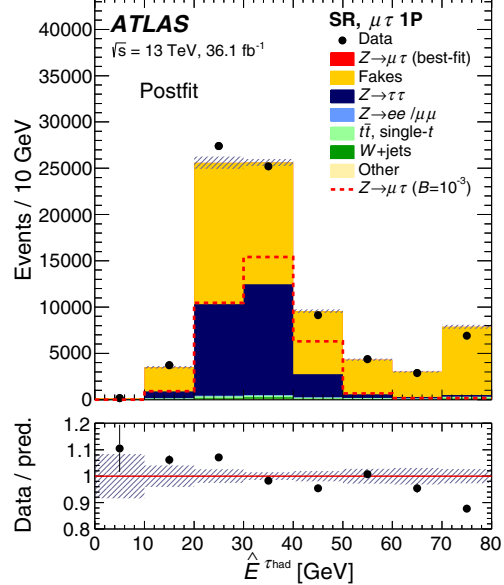

(b) $\hat{E}^{\tau_{\text {had }}}$

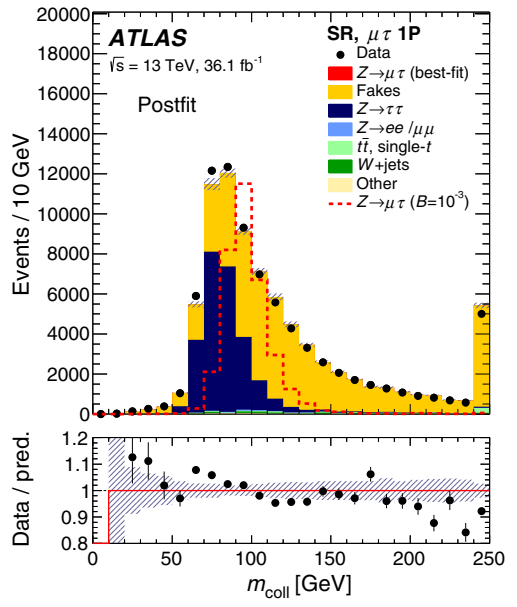

(e) $m_{\text {coll }}$

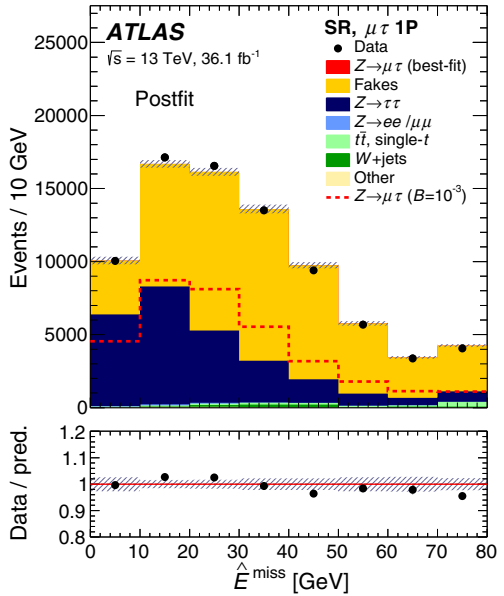

(c) $\hat{E}^{\text {miss }}$

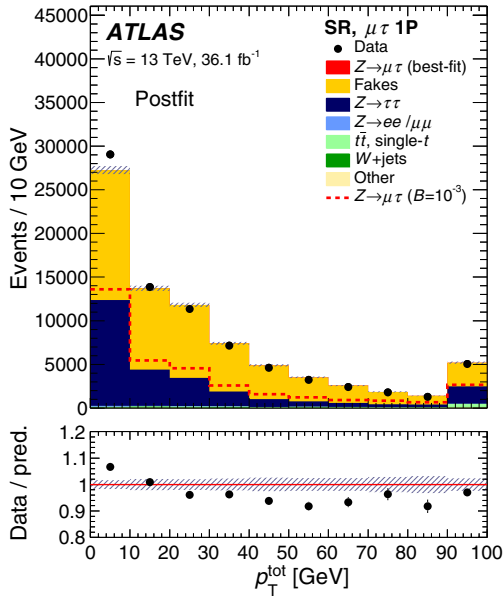

(f) $p_{\mathrm{T}}^{\text {tot }}$

FIG. 7. Observed and expected postfit distributions of unscaled NN inputs in SR for the $\mu \tau$ channel with 1-prong $\tau_{\text {had-vis }}$ candidates. The fit is based on profiling on the combined NN classifier, but not directly on these variables. The filled histogram stacked on top of the backgrounds represents the signal normalized to the best-fit $\mathcal{B}(Z \rightarrow \ell \tau)$. The overlaid dashed line represents the expected distribution for the signal normalized to $\mathcal{B}(Z \rightarrow \ell \tau)=10^{-3}$. In the panels below each plot, the ratios of the observed data (dots) and the postfit background plus signal (solid line) to the postfit background are shown. The hatched error bands represent the combined statistical and systematic uncertainties. The first and last bins include underflow and overflow events, respectively.

The result of the search for $Z \rightarrow \mu \tau$ decays presented here is combined with the result published by ATLAS with $20.3 \mathrm{fb}^{-1}$ of data at a center-of-mass energy of $\sqrt{s}=$ $8 \mathrm{TeV}$ [7]. In this previous analysis, a 95\% C.L. upper limit was set at $\mathcal{B}(Z \rightarrow \mu \tau)<1.7 \times 10^{-5}$. The expected upper limit was $2.6 \times 10^{-5}$.

The analysis of the $8 \mathrm{TeV}$ data was based on a template fit to the observed distributions in data of the $m_{\tau \mu}^{\mathrm{MMC}}$ mass, as reconstructed by using the missing mass calculator [56]. This is a likelihood-based mass estimator optimized for $Z \rightarrow \tau \tau$ events. The dominant irreducible $Z \rightarrow \tau \tau$ background was estimated using so-called embedded events [57] and was normalized to data. The reducible background of events with $\tau_{\text {had-vis }}$ candidates originating from misidentified jets was also estimated from data using events with $\mu \tau$ pairs with the same electric charges. The other smaller background contributions were estimated from simulation. The $Z \rightarrow \mu \tau$ signal was simulated and was normalized using the predicted $Z$ production cross section at $8 \mathrm{TeV}$.

The $8 \mathrm{TeV}$ and $13 \mathrm{TeV}$ analyses are combined using the same parameter of interest, but assuming no other correlation. Indeed, the estimates of the two dominant sources of background, $Z \rightarrow \tau \tau$ and fakes, are based on different data and different methods. The signal predictions are also uncorrelated since the $Z$ production cross section is either predicted, in the $8 \mathrm{TeV}$ analysis, or determined from data, in the $13 \mathrm{TeV}$ analysis. Furthermore, the systematic uncertainties related to the detector modeling in simulated data are typically based on auxiliary measurements performed 


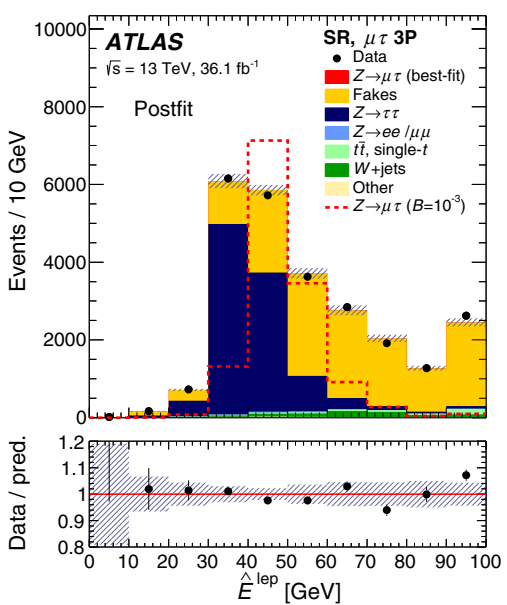

(a) $\hat{E}^{\text {lep }}$

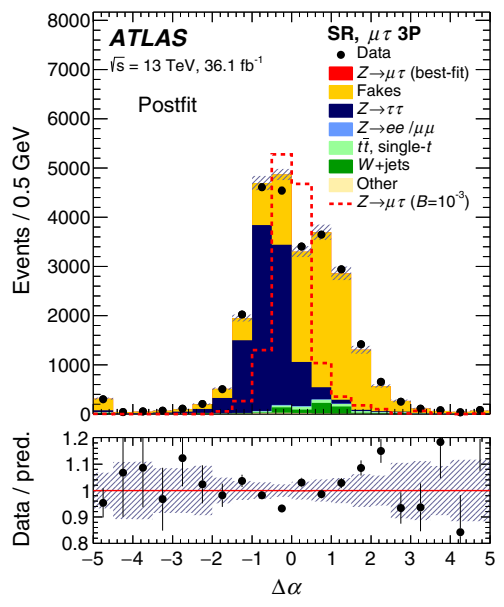

(d) $\Delta \alpha$

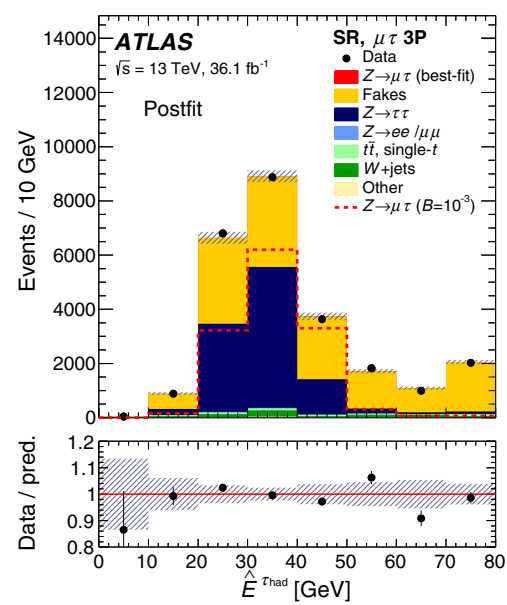

(b) $\hat{E}^{\tau_{\text {had }}}$

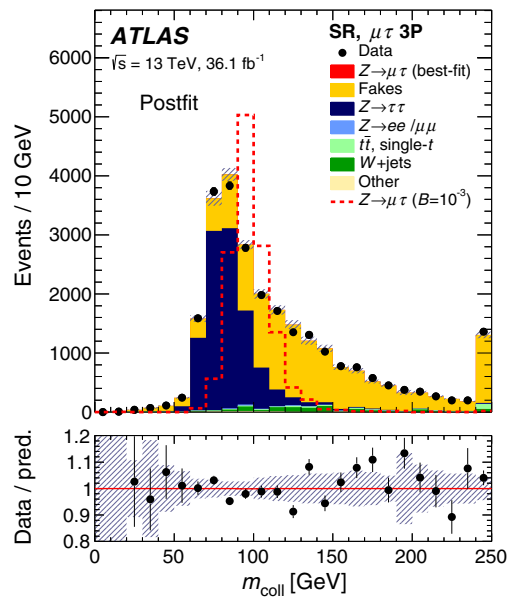

(e) $m_{\text {coll }}$

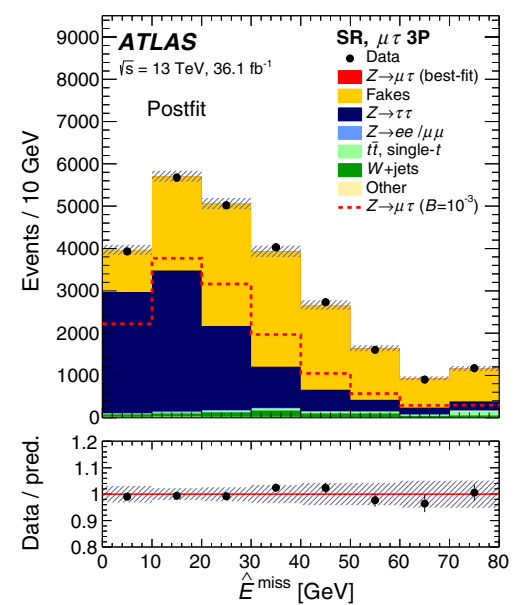

(c) $\hat{E}^{\text {miss }}$

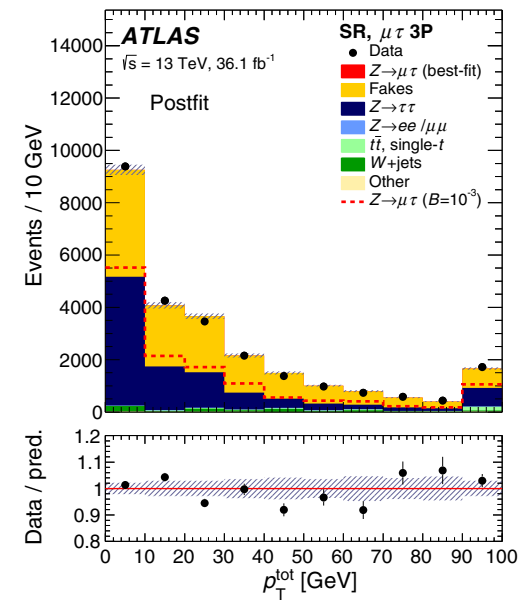

(f) $p_{\mathrm{T}}^{\text {tot }}$

FIG. 8. Observed and expected postfit distributions of unscaled NN inputs in SR for the $\mu \tau$ channel with 3-prong $\tau_{\text {had-vis }}$ candidates. The fit is based on profiling on the combined NN classifier, but not directly on these variables. The filled histogram stacked on top of the backgrounds represents the signal normalized to the best-fit $\mathcal{B}(Z \rightarrow \ell \tau)$. The overlaid dashed line represents the expected distribution for the signal normalized to $\mathcal{B}(Z \rightarrow \ell \tau)=10^{-3}$. In the panels below each plot, the ratios of the observed data (dots) and the postfit background plus signal (solid line) to the postfit background are shown. The hatched error bands represent the combined statistical and systematic uncertainties. The first and last bins include underflow and overflow events, respectively.

on different data. If these modeling uncertainties are set to zero, the combined upper limit changes by only $3 \%$. This $3 \%$ represents an upper bound on how much the combined limit can change if different assumptions are made about correlations in systematic uncertainties related to detector modeling.

The combined best-fit value of $\mathcal{B}(Z \rightarrow \mu \tau)$ is $\left(-0.8_{-0.8}^{+0.9}\right) \times 10^{-5}$ and the combined observed (expected) $95 \%$ C.L. upper limit is $\mathcal{B}(Z \rightarrow \mu \tau)<1.3(1.8) \times 10^{-5}$.

\section{CONCLUSIONS}

Direct searches for lepton flavor violation in decays of the $Z$ boson are performed using a data sample of protonproton collisions recorded by the ATLAS detector at the LHC corresponding to an integrated luminosity of $36.1 \mathrm{fb}^{-1}$ at a center-of-mass energy of $\sqrt{s}=13 \mathrm{TeV}$. The analysis selects events consistent with the decay of a $Z$ boson into an electron or muon and a hadronically decaying $\tau$ lepton. In these decays the $\tau$ lepton is assumed to be unpolarized. Neural network classifiers are used to discriminate signal from backgrounds, and the NN output distributions are analyzed in a template fit to data.

No significant excess of events above the expected background is observed and upper limits on the leptonflavor-violating branching ratios are set at the $95 \%$ confidence level using the $\mathrm{CL}_{\mathrm{s}}$ method: $\mathcal{B}(Z \rightarrow \mu \tau)<2.4 \times 10^{-5}$ and $\mathcal{B}(Z \rightarrow e \tau)<5.8 \times 10^{-5}$. The corresponding expected upper limits are $2.4 \times 10^{-5}$ and $2.8 \times 10^{-5}$, respectively. An excess of data over the expected backgrounds is 
observed in the $e \tau$ final state with a significance of $2.3 \sigma$.

No upper limits on $\mathcal{B}(Z \rightarrow e \tau)$ from ATLAS data have been published previously. The current best upper limit is from LEP at $\mathcal{B}(Z \rightarrow e \tau)<0.98 \times 10^{-5}$.

The result on $\mathcal{B}(Z \rightarrow \mu \tau)$ presented here is combined with the previous ATLAS result based on $20.3 \mathrm{fb}^{-1}$ of data at a center-of-mass energy of $\sqrt{s}=8 \mathrm{TeV}$. The combined 95\% C.L. upper limit is $\mathcal{B}(Z \rightarrow \mu \tau)<1.3 \times 10^{-5}$, to be compared with LEP upper limit of $\mathcal{B}(Z \rightarrow \mu \tau)<$ $1.2 \times 10^{-5}$.

\section{ACKNOWLEDGMENTS}

We thank CERN for the very successful operation of the LHC, as well as the support staff from our institutions without whom ATLAS could not be operated efficiently. We acknowledge the support of ANPCyT, Argentina; YerPhI, Armenia; ARC, Australia; BMWFW and FWF, Austria; ANAS, Azerbaijan; SSTC, Belarus; CNPq and FAPESP, Brazil; NSERC, NRC and CFI, Canada; CERN; CONICYT, Chile; CAS, MOST and NSFC, China; COLCIENCIAS, Colombia; MSMT CR, MPO CR and VSC CR, Czech Republic; DNRF and DNSRC, Denmark; IN2P3-CNRS, CEA-DRF/IRFU, France; SRNSFG, Georgia; BMBF, HGF, and MPG, Germany; GSRT, Greece; RGC, Hong Kong SAR, China; ISF, I-CORE and Benoziyo Center, Israel; INFN, Italy; MEXT and JSPS, Japan; CNRST, Morocco; NWO, Netherlands; RCN, Norway; MNiSW and NCN, Poland; FCT,
Portugal; MNE/IFA, Romania; MES of Russia and NRC KI, Russian Federation; JINR; MESTD, Serbia; MSSR, Slovakia; ARRS and MIZŠ, Slovenia; DST/NRF, South Africa; MINECO, Spain; SRC and Wallenberg Foundation, Sweden; SERI, SNSF and Cantons of Bern and Geneva, Switzerland; MOST, Taiwan; TAEK, Turkey; STFC, United Kingdom; DOE and NSF, United States of America. In addition, individual groups and members have received support from BCKDF, the Canada Council, CANARIE, CRC, Compute Canada, FQRNT, and the Ontario Innovation Trust, Canada; EPLANET, ERC, ERDF, FP7, Horizon 2020 and Marie Skłodowska-Curie Actions, European Union; Investissements d'Avenir Labex and Idex, ANR, Région Auvergne and Fondation Partager le Savoir, France; DFG and AvH Foundation, Germany; Herakleitos, Thales and Aristeia programmes co-financed by EU-ESF and the Greek NSRF; BSF, GIF and Minerva, Israel; BRF, Norway; CERCA Programme Generalitat de Catalunya, Generalitat Valenciana, Spain; the Royal Society and Leverhulme Trust, United Kingdom. The crucial computing support from all WLCG partners is acknowledged gratefully, in particular from CERN, the ATLAS Tier-1 facilities at TRIUMF (Canada), NDGF (Denmark, Norway, Sweden), CC-IN2P3 (France), KIT/ GridKA (Germany), INFN-CNAF (Italy), NL-T1 (Netherlands), PIC (Spain), ASGC (Taiwan), RAL (UK) and BNL (USA), the Tier-2 facilities worldwide and large non-WLCG resource providers. Major contributors of computing resources are listed in Ref. [58].
[1] J. I. Illana, M. Jack, and T. Riemann, Predictions for $Z \rightarrow \mu \tau$ and related reactions, arXiv:hep-ph/0001273.

[2] J. I. Illana and T. Riemann, Charged lepton flavor violation from massive neutrinos in $\mathrm{Z}$ decays, Phys. Rev. D 63, 053004 (2001).

[3] T.-K. Kuo and N. Nakagawa, Lepton flavor violating decays of $Z^{0}$ and $\tau$, Phys. Rev. D 32, 306 (1985).

[4] F. Gabbiani, J.H. Kim, and A. Masiero, $Z^{0} \rightarrow b \bar{s}$ and $Z^{0} \rightarrow \tau \bar{\mu}$ in SUSY: Are they observable? Phys. Lett. B 214, 398 (1988).

[5] R. Akers and G. Alexander (OPAL Collaboration), A search for lepton flavour violating $Z^{0}$ decays, Z. Phys. C 67, 555 (1995).

[6] DELPHI Collaboration, Search for lepton flavour number violating $Z^{0}$ - decays, Z. Phys. C 73, 243 (1997).

[7] ATLAS Collaboration, Search for lepton-flavour-violating decays of the Higgs and $Z$ bosons with the ATLAS detector, Eur. Phys. J. C 77, 70 (2017).

[8] CMS Collaboration, Search for Lepton flavor violation in Z and Higgs decays with the CMS Experiment, Nucl. Part. Phys. Proc. 287-288, 160 (2017).
[9] ATLAS Collaboration, Search for the lepton flavor violating decay $Z \rightarrow e \mu$ in $p p$ collisions at $\sqrt{s}=8 \mathrm{TeV}$ with the ATLAS detector, Phys. Rev. D 90, 072010 (2014).

[10] ATLAS Collaboration, The ATLAS experiment at the CERN large hadron collider, J. Instrum. 3, S08003 (2008).

[11] ATLAS Collaboration, ATLAS insertable B-layer technical design report, CERN Report No. ATLAS-TDR-19, 2010, https://cds.cern.ch/record/1291633; Addendum, CERN Report. No. ATLAS-TDR-19-ADD-1, 2012, https://cds .cern.ch/record/1451888.

[12] ATLAS Collaboration, Performance of the ATLAS trigger system in 2015, Eur. Phys. J. C 77, 317 (2017).

[13] ATLAS Collaboration, Electron efficiency measurements with the ATLAS detector using the 2015 LHC protonproton collision data, CERN Report No. ATLAS-CONF2016-024, 2016, https://cds.cern.ch/record/2157687.

[14] ATLAS Collaboration, Muon reconstruction performance of the ATLAS detector in proton-proton collision data at $\sqrt{s}=13$ TeV, Eur. Phys. J. C 76, 292 (2016).

[15] M. Cacciari, G. P. Salam, and G. Soyez, The anti- $k_{t}$ jet clustering algorithm, J. High Energy Phys. 04 (2008) 063. 
[16] M. Cacciari, G. P. Salam, and G. Soyez, FastJet user manual, Eur. Phys. J. C 72, 1896 (2012).

[17] ATLAS Collaboration, Jet energy scale measurements and their systematic uncertainties in proton-proton collisions at $\sqrt{s}=13 \mathrm{TeV}$ with the ATLAS detector, Phys. Rev. D 96, 072002 (2017).

[18] ATLAS Collaboration, Identification and rejection of pileup jets at high pseudorapidity with the ATLAS detector, Eur. Phys. J. C 77, 580 (2017).

[19] ATLAS Collaboration, Vertex reconstruction performance of the ATLAS detector at $\sqrt{s}=13 \mathrm{TeV}$, CERN Report No. ATL-PHYS-PUB-2015-026, 2015, https://cds.cern.ch/ record/2037717.

[20] ATLAS Collaboration, Optimisation of the ATLAS btagging performance for the 2016 LHC run, CERN Report No. ATL-PHYS-PUB-2016-012, 2016, https://cds.cern.ch/ record/2160731.

[21] C. Patrignani et al., Review of particle physics, Chin. Phys. C 40, 100001 (2016).

[22] ATLAS Collaboration, Identification and energy calibration of hadronically decaying tau leptons with the ATLAS experiment in $p p$ collisions at $\sqrt{s}=8 \mathrm{TeV}$, Eur. Phys. J. C 75, 303 (2015).

[23] ATLAS Collaboration, Reconstruction, energy calibration, and identification of hadronically decaying tau leptons in the ATLAS experiment for Run-2 of the LHC, CERN Report No. ATL-PHYS-PUB-2015-045, 2015, https://atlas .web.cern.ch/Atlas/GROUPS/PHYSICS/PUBNOTES/ATLPHYS-PUB-2015-045.

[24] ATLAS Collaboration, Measurement of the tau lepton reconstruction and identification performance in the ATLAS experiment using $p p$ collisions at $\sqrt{s}=13 \mathrm{TeV}, \mathrm{CERN}$ Report No. ATLAS-CONF-2017-029, 2017, https://cds .cern.ch/record/2261772.

[25] ATLAS Collaboration, Performance of missing transverse momentum reconstruction with the ATLAS detector using proton-proton collisions at $\sqrt{s}=13 \mathrm{TeV}$, arXiv: 1802.08168.

[26] ATLAS Collaboration, Luminosity determination in $p p$ collisions at $\sqrt{s}=8 \mathrm{TeV}$ using the ATLAS detector at the LHC, Eur. Phys. J. C 76, 653 (2016).

[27] T. Sjostrand, S. Mrenna, and P.Z. Skands, A brief introduction to PYTHIA 8.1, Comput. Phys. Commun. 178, 852 (2008).

[28] R. D. Ball et al., Parton distributions with LHC data, Nucl. Phys. B867, 244 (2013).

[29] ATLAS Collaboration, ATLAS Pythia 8 tunes to $7 \mathrm{TeV}$ data, CERN Report No. ATL-PHYS-PUB-2014-021, 2014, https://cds.cern.ch/record/1966419.

[30] K. Melnikov and F. Petriello, Electroweak gauge boson production at hadron colliders through $\mathcal{O}\left(\alpha_{s}^{2}\right)$, Phys. Rev. D 74, 114017 (2006).

[31] T. Gleisberg, S. Höche, F. Krauss, M. Schönherr, S. Schumann, F. Siegert, and J. Winter, Event generation with SHERPA 1.1, J. High Energy Phys. 02 (2009) 007.

[32] R. D. Ball et al., Parton distributions for the LHC Run II, J. High Energy Phys. 04 (2015) 040.

[33] T. Gleisberg and S. Hoeche, Comix, a new matrix element generator, J. High Energy Phys. 12 (2008) 039.
[34] F. Cascioli, P. Maierhofer, and S. Pozzorini, Scattering Amplitudes with Open Loops, Phys. Rev. Lett. 108, 111601 (2012).

[35] S. Hoeche, F. Krauss, M. Schonherr, and F. Siegert, QCD matrix elements + parton showers: The NLO case, J. High Energy Phys. 04 (2013) 027.

[36] S. Alioli, P. Nason, C. Oleari, and E. Re, A general framework for implementing NLO calculations in shower Monte Carlo programs: The POWHEG BOX, J. High Energy Phys. 06 (2010) 043.

[37] P. Nason, A new method for combining NLO QCD with shower Monte Carlo algorithms, J. High Energy Phys. 11 (2004) 040.

[38] S. Frixione, P. Nason, and C. Oleari, Matching NLO QCD computations with Parton Shower simulations: The POWHEG method, J. High Energy Phys. 11 (2007) 070.

[39] H.-L. Lai, M. Guzzi, J. Huston, Z. Li, P. M. Nadolsky, J. Pumplin, and C.-P. Yuan, New parton distributions for collider physics, Phys. Rev. D 82, 074024 (2010).

[40] ATLAS Collaboration, Measurement of the $Z / \gamma^{*}$ boson transverse momentum distribution in $p p$ collisions at $\sqrt{s}=$ $7 \mathrm{TeV}$ with the ATLAS detector, J. High Energy Phys. 09 (2014) 145.

[41] T. Sjostrand, S. Mrenna, and P.Z. Skands, PYTHIA 6.4 physics and manual, J. High Energy Phys. 05 (2006) 026.

[42] P. Z. Skands, Tuning Monte Carlo generators: The Perugia tunes, Phys. Rev. D 82, 074018 (2010).

[43] D. J. Lange, The EvtGen particle decay simulation package, Nucl. Instrum. Methods Phys. Res., Sect. A 462, 152 (2001).

[44] ATLAS Collaboration, Summary of ATLAS Pythia 8 tunes, CERN Report No. ATL-PHYS-PUB-2012-003, 2012, https://cds.cern.ch/record/1474107.

[45] A. D. Martin, W. J. Stirling, R. S. Thorne, and G. Watt, Parton distributions for the LHC, Eur. Phys. J. C 63, 189 (2009).

[46] S. Agostinelli et al., GEANT4: A simulation toolkit, Nucl. Instrum. Methods Phys. Res., Sect. A 506, 250 (2003).

[47] ATLAS Collaboration, The ATLAS simulation infrastructure, Eur. Phys. J. C 70, 823 (2010).

[48] S. Davidson, S. Lacroix, and P. Verdier, LHC sensitivity to lepton flavour violating $\mathrm{Z}$ boson decays, J. High Energy Phys. 09 (2012) 092.

[49] Keras, https://keras.io/.

[50] M. Abadi et al., TensorFlow: Large-acale machine learning on heterogeneous systems, Software available from tensorflow.org, 2015, https://www.tensorflow.org/.

[51] W. Verkerke and D. P. Kirkby, The RooFit toolkit for data modeling, eConf C0303241, MOLT007 (2003).

[52] L. Moneta, K. Cranmer, G. Schott, and W. Verkerke, The RooStats project, Proc. Sci., ACAT2010 (2010) 057.

[53] M. Baak, G. J. Besjes, D. Côté, A. Koutsman, J. Lorenz, and D. Short, HistFitter software framework for statistical data analysis, Eur. Phys. J. C. 75, 153 (2015).

[54] G. Cowan, K. Cranmer, E. Gross, and O. Vitells, Asymptotic formulae for likelihood-based tests of new physics, Eur. Phys. J. C 71, 1554 (2011); Erratum, Eur. Phys. J. C73, 2501(E) (2013). 
[55] A. L. Read, Presentation of search results: The CLs technique, J. Phys. G 28, 2693 (2002).

[56] A. Elagin, P. Murat, A. Pranko, and A. Safonov, A new mass reconstruction technique for resonances decaying to $\tau \tau$, Nucl. Instrum. Methods Phys. Res., Sect. A 654, 481 (2011).
[57] ATLAS Collaboration, Modelling $Z \rightarrow \tau \tau$ processes in ATLAS with $\tau$-embedded $Z \rightarrow \mu \mu$ data, J. Instrum. 10, P09018 (2015).

[58] ATLAS Collaboration, ATLAS computing acknowledgements 2016-2017, CERN Report No. ATL-GEN-PUB2016-002, 2016, https://cds.cern.ch/record/2202407.

M. Aaboud ${ }^{34 d}$ G. Aad,${ }^{99}$ B. Abbott, ${ }^{124}$ O. Abdinov, ${ }^{13, a}$ B. Abeloos, ${ }^{128}$ D. K. Abhayasinghe, ${ }^{91}$ S. H. Abidi, ${ }^{164}$ O. S. AbouZeid, ${ }^{143}$ N. L. Abraham, ${ }^{153}$ H. Abramowicz, ${ }^{158}$ H. Abreu, ${ }^{157}$ Y. Abulaiti, ${ }^{6}$ B. S. Acharya, ${ }^{64 a, 64 b, b}$ S. Adachi, ${ }^{160}$ L. Adamczyk, ${ }^{81 \mathrm{a}} \mathrm{J}$. Adelman, ${ }^{119}$ M. Adersberger, ${ }^{112}$ A. Adiguzel, ${ }^{12 c, c}$ T. Adye ${ }^{141}$ A. A. Affolder, ${ }^{143}$ Y. Afik, ${ }^{157}$ C. Agheorghiesei ${ }^{27 \mathrm{c}}$ J. A. Aguilar-Saavedra, ${ }^{136 f, 136 \mathrm{a}}$ F. Ahmadov, ${ }^{77, d}$ G. Aielli, ${ }^{71 a, 71 b}$ S. Akatsuka, ${ }^{83}$ T. P. A. Åkesson, ${ }^{94}$ E. Akilli, ${ }^{52}$ A. V. Akimov, ${ }^{108}$ G. L. Alberghi, ${ }^{23 b, 23 a}$ J. Albert,${ }^{173}$ P. Albicocco, ${ }^{49}$ M. J. Alconada Verzini,${ }^{86}$ S. Alderweireldt, ${ }^{117}$ M. Aleksa, ${ }^{35}$ I. N. Aleksandrov, ${ }^{77}$ C. Alexa, ${ }^{27 b}$ T. Alexopoulos, ${ }^{10}$ M. Alhroob, ${ }^{124}$ B. Ali, ${ }^{138}$ G. Alimonti, ${ }^{66 a}$ J. Alison, ${ }^{36}$ S. P. Alkire, ${ }^{145}$ C. Allaire, ${ }^{128}$ B. M. M. Allbrooke, ${ }^{153}$ B. W. Allen, ${ }^{127}$ P. P. Allport, ${ }^{21}$ A. Aloisio, ${ }^{67 a, 67 b}$ A. Alonso, ${ }^{39}$ F. Alonso, ${ }^{86}$ C. Alpigiani, ${ }^{145}$ A. A. Alshehri, ${ }^{55}$ M. I. Alstaty, ${ }^{99}$ B. Alvarez Gonzalez,${ }^{35}$ D. Álvarez Piqueras, ${ }^{171}$ M. G. Alviggi, ${ }^{67 a, 67 b}$ B. T. Amadio, ${ }^{18}$ Y. Amaral Coutinho, ${ }^{78 b}$ L. Ambroz, ${ }^{131}$ C. Amelung, ${ }^{26}$ D. Amidei, ${ }^{103}$ S. P. Amor Dos Santos, ${ }^{136 a, 136 c}$ S. Amoroso, ${ }^{35}$ C. S. Amrouche,${ }^{52}$ C. Anastopoulos, ${ }^{146}$ L. S. Ancu, ${ }^{52}$ N. Andari, ${ }^{21}$ T. Andeen, ${ }^{11}$ C. F. Anders, ${ }^{59 b}$ J. K. Anders, ${ }^{20}$ K. J. Anderson, ${ }^{36}$ A. Andreazza, ${ }^{66 a, 66 b}$ V. Andrei,${ }^{59 a}$ C. R. Anelli, ${ }^{173}$ S. Angelidakis, ${ }^{37}$ I. Angelozzi, ${ }^{118}$ A. Angerami, ${ }^{38}$ A. V. Anisenkov, ${ }^{120 b, 120 a}$ A. Annovi, ${ }^{69 a}$ C. Antel,${ }^{59 a}$ M. T. Anthony, ${ }^{146}$ M. Antonelli, ${ }^{49}$ D. J. A. Antrim, ${ }^{168}$ F. Anulli, ${ }^{70 a}$ M. Aoki, ${ }^{79}$ L. Aperio Bella, ${ }^{35}$ G. Arabidze,${ }^{104}$ Y. Arai, ${ }^{79}$ J. P. Araque, ${ }^{136 \mathrm{a}}$ V. Araujo Ferraz ${ }^{78 b}$ R. Araujo Pereira, ${ }^{78 b}$ A. T. H. Arce, ${ }^{47}$ R. E. Ardell, ${ }^{91}$ F. A. Arduh ${ }^{86}$ J-F. Arguin, ${ }^{107}$ S. Argyropoulos, ${ }^{75}$ A. J. Armbruster, ${ }^{35}$ L. J. Armitage, ${ }^{90}$ A. Armstrong, ${ }^{168}$ O. Arnaez, ${ }^{164}$ H. Arnold, ${ }^{118}$ M. Arratia, ${ }^{31}$ O. Arslan, ${ }^{24}$ A. Artamonov, ${ }^{109, a}$ G. Artoni, ${ }^{131}$ S. Artz, ${ }^{97}$ S. Asai,${ }^{160}$ N. Asbah, ${ }^{44}$ A. Ashkenazi, ${ }^{158}$ E. M. Asimakopoulou, ${ }^{169}$ L. Asquith, ${ }^{153}$ K. Assamagan, ${ }^{29}$ R. Astalos ${ }^{28 a}$ R. J. Atkin, ${ }^{32 a}$ M. Atkinson, ${ }^{170}$ N. B. Atlay, ${ }^{148}$ K. Augsten, ${ }^{138}$ G. Avolio, ${ }^{35}$ R. Avramidou, ${ }^{58 a}$ B. Axen, ${ }^{18}$ M. K. Ayoub, ${ }^{15 a}$ G. Azuelos, ${ }^{107, e}$ A. E. Baas, ${ }^{59 a}$ M. J. Baca, ${ }^{21}$ H. Bachacou, ${ }^{142}$ K. Bachas, ${ }^{65 a, 65 b}$ M. Backes, ${ }^{131}$ P. Bagnaia, ${ }^{70 a, 70 b}$ M. Bahmani, ${ }^{82}$ H. Bahrasemani, ${ }^{149}$ A. J. Bailey, ${ }^{171}$ J. T. Baines, ${ }^{141}$ M. Bajic, ${ }^{39}$ C. Bakalis,${ }^{10}$ O. K. Baker, ${ }^{180}$ P. J. Bakker, ${ }^{118}$ D. Bakshi Gupta, ${ }^{93}$ E. M. Baldin, ${ }^{120 b, 120 a}$ P. Balek, ${ }^{177}$ F. Balli, ${ }^{142}$ W. K. Balunas, ${ }^{133}$ J. Balz, ${ }^{97}$ E. Banas, ${ }^{82}$ A. Bandyopadhyay, ${ }^{24}$ S. Banerjee,${ }^{178, f}$ A. A. E. Bannoura, ${ }^{179}$ L. Barak, ${ }^{158}$ W. M. Barbe,${ }^{37}$ E. L. Barberio, ${ }^{102}$ D. Barberis, ${ }^{53 b, 53 a}$ M. Barbero, ${ }^{99}$ T. Barillari, ${ }^{113}$ M-S. Barisits, ${ }^{35}$ J. Barkeloo, ${ }^{127}$ T. Barklow, ${ }^{150}$ N. Barlow,${ }^{31}$ R. Barnea, ${ }^{157}$ S. L. Barnes, ${ }^{58 c}$ B. M. Barnett, ${ }^{141}$ R. M. Barnett, ${ }^{18}$ Z. Barnovska-Blenessy,${ }^{58 a}$ A. Baroncelli, ${ }^{72 a}$ G. Barone, ${ }^{26}$ A. J. Barr, ${ }^{131}$ L. Barranco Navarro, ${ }^{171}$ F. Barreiro, ${ }^{96}$ J. Barreiro Guimarães da Costa ${ }^{15 a}$ R. Bartoldus, ${ }^{150}$ A. E. Barton, ${ }^{87}$ P. Bartos, ${ }^{28 a}$ A. Basalaev, ${ }^{134}$ A. Bassalat, ${ }^{128}$ R. L. Bates,${ }^{55}$ S. J. Batista, ${ }^{164}$ S. Batlamous, ${ }^{34 \mathrm{e}}$ J. R. Batley,${ }^{31}$ M. Battaglia, ${ }^{143}$ M. Bauce, ${ }^{70 a}, 70 \mathrm{~b}$ F. Bauer, ${ }^{142}$ K. T. Bauer, ${ }^{168}$ H. S. Bawa, ${ }^{150, g}$ J. B. Beacham, ${ }^{122}$ M. D. Beattie,${ }^{87}$ T. Beau, ${ }^{132}$ P. H. Beauchemin, ${ }^{167}$ P. Bechtle, ${ }^{24}$ H. C. Beck, ${ }^{51}$ H. P. Beck, ${ }^{20, \mathrm{~h}}$ K. Becker, ${ }^{50}$ M. Becker, ${ }^{97}$ C. Becot, ${ }^{44}$ A. Beddall, ${ }^{12 \mathrm{~d}}$ A. J. Beddall, ${ }^{12 \mathrm{a}}$ V. A. Bednyakov, ${ }^{77}$ M. Bedognetti, ${ }^{118}$ C. P. Bee, ${ }^{152}$ T. A. Beermann, ${ }^{35}$ M. Begalli, ${ }^{78 b}$ M. Begel, ${ }^{29}$ A. Behera, ${ }^{152}$ J. K. Behr, ${ }^{44}$ A. S. Bell,${ }^{92}$ G. Bella, ${ }^{158}$ L. Bellagamba, ${ }^{23 b}$ A. Bellerive, ${ }^{33}$ M. Bellomo, ${ }^{157}$ P. Bellos, ${ }^{9}$ K. Belotskiy, ${ }^{110}$ N. L. Belyaev, ${ }^{110}$ O. Benary, ${ }^{158, a}$ D. Benchekroun, ${ }^{34 a}$ M. Bender, ${ }^{12}$ N. Benekos, ${ }^{10}$ Y. Benhammou, ${ }^{158}$ E. Benhar Noccioli, ${ }^{180}$ J. Benitez, ${ }^{75}$ D. P. Benjamin, ${ }^{47}$ M. Benoit, ${ }^{52}$ J. R. Bensinger, ${ }^{26}$ S. Bentvelsen, ${ }^{118}$ L. Beresford, ${ }^{131}$ M. Beretta, ${ }^{49}$ D. Berge, ${ }^{44}$ E. Bergeaas Kuutmann, ${ }^{169}$ N. Berger, ${ }^{5}$ L. J. Bergsten, ${ }^{26}$ J. Beringer, ${ }^{18}$ S. Berlendis, ${ }^{7}$ N. R. Bernard,${ }^{100}$ G. Bernardi, ${ }^{132}$ C. Bernius, ${ }^{150}$ F. U. Bernlochner ${ }^{24}$ T. Berry, ${ }^{91}$ P. Berta, ${ }^{97}$ C. Bertella, ${ }^{15 a}$ G. Bertoli, ${ }^{43 a}{ }^{43 b}$ I. A. Bertram, ${ }^{87}$ G. J. Besjes,${ }^{39}$ O. Bessidskaia Bylund, ${ }^{43 a, 43 b}$ M. Bessner,${ }^{44}$ N. Besson, ${ }^{142}$ A. Bethani, ${ }^{98}$ S. Bethke, ${ }^{113}$ A. Betti, ${ }^{24}$ A. J. Bevan, ${ }^{90}$ J. Beyer, ${ }^{113}$ R. M. Bianchi, ${ }^{135}$ O. Biebel, ${ }^{112}$ D. Biedermann, ${ }^{19}$ R. Bielski, ${ }^{98}$ K. Bierwagen, ${ }^{97}$ N. V. Biesuz, ${ }^{69 a, 69 b}$ M. Biglietti, ${ }^{72 a}$ T. R. V. Billoud, ${ }^{107}$ M. Bindi, ${ }^{51}$ A. Bingul, ${ }^{12 \mathrm{~d}}$ C. Bini, ${ }^{70 a, 70 b}$ S. Biondi, ${ }^{23 b, 23 \mathrm{a}}$ T. Bisanz, ${ }^{51}$ J. P. Biswal, ${ }^{158}$ C. Bittrich, ${ }^{46}$ D. M. Bjergaard, ${ }^{47}$ J. E. Black, ${ }^{150}$ K. M. Black ${ }^{25}$ R. E. Blair, ${ }^{6}$ T. Blazek, ${ }^{28 a}$ I. Bloch, ${ }^{44}$ C. Blocker, ${ }^{26}$ A. Blue,${ }^{55}$ U. Blumenschein, ${ }^{90}$ Dr. Blunier, ${ }^{144 a}$ G. J. Bobbink, ${ }^{118}$ V. S. Bobrovnikov, ${ }^{120 b, 120 a}$ S. S. Bocchetta, ${ }^{94}$ A. Bocci, ${ }^{47}$ D. Boerner, ${ }^{179}$ D. Bogavac, ${ }^{112}$ A. G. Bogdanchikov, ${ }^{120 b, 120 a}$ C. Bohm, ${ }^{43 a}$ V. Boisvert, ${ }^{91}$ P. Bokan, ${ }^{169,51}$ T. Bold, ${ }^{81 a}$ A. S. Boldyrev, ${ }^{111}$ A. E. Bolz,${ }^{59 b}$ M. Bomben,${ }^{132}$ M. Bona,${ }^{90}$ J. S. Bonilla,${ }^{127}$ M. Boonekamp, ${ }^{142}$ A. Borisov,${ }^{140}$ G. Borissov, ${ }^{87}$ J. Bortfeldt, ${ }^{35}$ D. Bortoletto, ${ }^{131}$ V. Bortolotto, ${ }^{71,61 b, 61 \mathrm{c}, 71 \mathrm{~b}}$ D. Boscherini, ${ }^{23 \mathrm{~b}}$ M. Bosman, ${ }^{14} \mathrm{~J}$. D. Bossio Sola, ${ }^{30}$ 
K. Bouaouda, ${ }^{34 a}$ J. Boudreau, ${ }^{135}$ E. V. Bouhova-Thacker, ${ }^{87}$ D. Boumediene, ${ }^{37}$ C. Bourdarios, ${ }^{128}$ S. K. Boutle,${ }^{55}$ A. Boveia, ${ }^{122}$ J. Boyd,${ }^{35}$ I. R. Boyko, ${ }^{77}$ A. J. Bozson, ${ }^{91}$ J. Bracinik, ${ }^{21}$ N. Brahimi, ${ }^{99}$ A. Brandt, ${ }^{8}$ G. Brandt, ${ }^{179}$ O. Brandt, ${ }^{59 a}$ F. Braren, ${ }^{44}$ U. Bratzler, ${ }^{161}$ B. Brau, ${ }^{100}$ J. E. Brau, ${ }^{127}$ W. D. Breaden Madden, ${ }^{55}$ K. Brendlinger, ${ }^{44}$ A. J. Brennan, ${ }^{102}$ L. Brenner, ${ }^{44}$ R. Brenner, ${ }^{169}$ S. Bressler, ${ }^{177}$ B. Brickwedde, ${ }^{97}$ D. L. Briglin, ${ }^{21}$ D. Britton, ${ }^{55}$ D. Britzger, ${ }^{59 b}$ I. Brock, ${ }^{24}$ R. Brock, ${ }^{104}$ G. Brooijmans, ${ }^{38}$ T. Brooks, ${ }^{91}$ W. K. Brooks, ${ }^{144 b}$ E. Brost,${ }^{119}$ J. H Broughton, ${ }^{21}$ P. A. Bruckman de Renstrom,${ }^{82}$ D. Bruncko, ${ }^{28 b}$ A. Bruni, ${ }^{23 b}$ G. Bruni, ${ }^{23 b}$ L. S. Bruni, ${ }^{118}$ S. Bruno, ${ }^{71 a, 71 b}$ B. H. Brunt, ${ }^{31}$ M. Bruschi, ${ }^{23 b}$ N. Bruscino, ${ }^{135}$ P. Bryant,${ }^{36}$ L. Bryngemark, ${ }^{44}$ T. Buanes, ${ }^{17}$ Q. Buat,${ }^{35}$ P. Buchholz, ${ }^{148}$ A. G. Buckley,${ }^{55}$ I. A. Budagov, ${ }^{77}$ F. Buehrer, ${ }^{50}$ M. K. Bugge, ${ }^{130}$ O. Bulekov, ${ }^{110}$ D. Bullock, ${ }^{8}$ T. J. Burch, ${ }^{119}$ S. Burdin, ${ }^{88}$ C. D. Burgard, ${ }^{118}$ A. M. Burger, ${ }^{5}$ B. Burghgrave, ${ }^{119}$ K. Burka, ${ }^{82}$ S. Burke, ${ }^{141}$ I. Burmeister, ${ }^{45}$ J. T. P. Burr, ${ }^{131}$ D. Büscher, ${ }^{50}$ V. Büscher, ${ }^{97}$ E. Buschmann, ${ }^{51}$ P. Bussey,${ }^{55}$ J. M. Butler, ${ }^{25}$ C. M. Buttar, ${ }^{55}$ J. M. Butterworth, ${ }^{92}$ P. Butti, ${ }^{35}$ W. Buttinger, ${ }^{35}$ A. Buzatu, ${ }^{155}$ A. R. Buzykaev, ${ }^{120 b, 120 a}$ G. Cabras, ${ }^{23 b, 23 a}$ S. Cabrera Urbán, ${ }^{171}$ D. Caforio, ${ }^{138}$ H. Cai, ${ }^{170}$ V. M. M. Cairo, ${ }^{2}$ O. Cakir, ${ }^{4 a}$ N. Calace, ${ }^{52}$ P. Calafiura, ${ }^{18}$ A. Calandri, ${ }^{99}$ G. Calderini, ${ }^{132}$ P. Calfayan, ${ }^{63}$ G. Callea, ${ }^{40 b, 40 a}$ L. P. Caloba,${ }^{78 b}$ S. Calvente Lopez, ${ }^{96}$ D. Calvet, ${ }^{37}$ S. Calvet,${ }^{37}$ T. P. Calvet, ${ }^{152}$ M. Calvetti, ${ }^{6 a, 69 b}$ R. Camacho Toro, ${ }^{132}$ S. Camarda, ${ }^{35}$ P. Camarri, ${ }^{71 a, 71 b}$ D. Cameron, ${ }^{130}$ R. Caminal Armadans, ${ }^{100}$ C. Camincher, ${ }^{35}$ S. Campana, ${ }^{35}$ M. Campanelli, ${ }^{92}$ A. Camplani, ${ }^{39}$ A. Campoverde, ${ }^{148}$ V. Canale,${ }^{67 a, 67 b}$ M. Cano Bret, ${ }^{58 c}$ J. Cantero, ${ }^{125}$ T. Cao ${ }^{158}$ Y. Cao, ${ }^{170}$ M. D. M. Capeans Garrido, ${ }^{35}$ I. Caprini, ${ }^{27 b}$ M. Caprini, ${ }^{27 b}$ M. Capua, ${ }^{40 b, 40 a}$ R. M. Carbone, ${ }^{38}$ R. Cardarelli, ${ }^{71 a}$ F. C. Cardillo,${ }^{50}$ I. Carli, ${ }^{139}$ T. Carli, ${ }^{35}$ G. Carlino, ${ }^{67 a}$ B. T. Carlson, ${ }^{135}$ L. Carminati, ${ }^{66 a, 66 b}$ R. M. D. Carney, ${ }^{43 a, 43 b}$ S. Caron, ${ }^{117}$ E. Carquin, ${ }^{144 b}$ S. Carrá, ${ }^{66 a, 66 b}$ G. D. Carrillo-Montoya, ${ }^{35}$ D. Casadei, ${ }^{32 b}$ M. P. Casado, ${ }^{14, i}$ A. F. Casha, ${ }^{164}$ M. Casolino, ${ }^{14}$ D. W. Casper, ${ }^{168}$ R. Castelijn, ${ }^{118}$ F. L. Castillo, ${ }^{171}$ V. Castillo Gimenez, ${ }^{171}$ N. F. Castro, ${ }^{136 a, 136 e}$ A. Catinaccio, ${ }^{35}$ J. R. Catmore, ${ }^{130}$ A. Cattai, ${ }^{35}$ J. Caudron, ${ }^{24}$ V. Cavaliere, ${ }^{29}$ E. Cavallaro, ${ }^{14}$ D. Cavalli, ${ }^{66 \mathrm{a}}$ M. Cavalli-Sforza ${ }^{14}$ V. Cavasinni, ${ }^{69,69 \mathrm{~b}}$ E. Celebi, ${ }^{12 \mathrm{~b}}$ F. Ceradini, ${ }^{72 a, 72 \mathrm{~b}}$ L. Cerda Alberich, ${ }^{171}$ A. S. Cerqueira, ${ }^{78 a}$ A. Cerri, ${ }^{153}$ L. Cerrito, ${ }^{71 a, 71 b}$ F. Cerutti, ${ }^{18}$ A. Cervelli, ${ }^{23 b, 23 a}$ S. A. Cetin, ${ }^{12 b}$ A. Chafaq, ${ }^{34 a}$ D. Chakraborty, ${ }^{119}$ S. K. Chan, ${ }^{57}$ W. S. Chan, ${ }^{118}$ Y. L. Chan, ${ }^{61 a}$ P. Chang, ${ }^{170}$ J. D. Chapman, ${ }^{31}$ D. G. Charlton, ${ }^{21}$ C. C. Chau, ${ }^{33}$ C. A. Chavez Barajas, ${ }^{153}$ S. Che, ${ }^{122}$ A. Chegwidden, ${ }^{104}$ S. Chekanov, ${ }^{6}$ S. V. Chekulaev, ${ }^{165 a}$ G. A. Chelkov, ${ }^{77, j}$ M. A. Chelstowska, ${ }^{35}$ C. Chen, ${ }^{58 a}$ C. H. Chen, ${ }^{76}$ H. Chen, ${ }^{29}$ J. Chen, ${ }^{58 a}$ J. Chen, ${ }^{38}$ S. Chen, ${ }^{133}$ S. J. Chen, ${ }^{15 c}$ X. Chen, ${ }^{15 b, k}$ Y. Chen, ${ }^{80}$ Y-H. Chen, ${ }^{44}$ H. C. Cheng, ${ }^{103}$ H. J. Cheng, ${ }^{15 \mathrm{~d}}$ A. Cheplakov, ${ }^{77}$ E. Cheremushkina, ${ }^{140}$ R. Cherkaoui El Moursli, ${ }^{34 \mathrm{e}}$ E. Cheu, ${ }^{7}$ K. Cheung, ${ }^{62}$ L. Chevalier, ${ }^{142}$ V. Chiarella, ${ }^{49}$ G. Chiarelli, ${ }^{69 a}$ G. Chiodini, ${ }^{65 a}$ A. S. Chisholm ${ }^{35}$ A. Chitan, ${ }^{27 b}$ I. Chiu, ${ }^{160}$ Y. H. Chiu, ${ }^{173}$ M. V. Chizhov, ${ }^{77}$ K. Choi, ${ }^{63}$ A. R. Chomont,${ }^{128}$ S. Chouridou, ${ }^{159}$ Y. S. Chow, ${ }^{118}$ V. Christodoulou, ${ }^{92}$ M. C. Chu, ${ }^{61 \mathrm{a}}$ J. Chudoba, ${ }^{137}$ A. J. Chuinard, ${ }^{101}$ J. J. Chwastowski, ${ }^{82}$ L. Chytka, ${ }^{126}$ D. Cinca, ${ }^{45}$ V. Cindro, ${ }^{89}$ I. A. Cioară, ${ }^{24}$ A. Ciocio, ${ }^{18}$ F. Cirotto, ${ }^{67 a, 67 b}$ Z. H. Citron, ${ }^{177}$ M. Citterio, ${ }^{66 a}$ A. Clark, ${ }^{52}$ M. R. Clark, ${ }^{38}$ P. J. Clark, ${ }^{48}$ C. Clement, ${ }^{43 a, 43 b}$ Y. Coadou, ${ }^{99}$ M. Cobal,${ }^{64 a, 64 c}$ A. Coccaro, ${ }^{53 b, 53 a}$ J. Cochran, ${ }^{76}$ A. E. C. Coimbra, ${ }^{177}$ L. Colasurdo, ${ }^{117}$ B. Cole, ${ }^{38}$ A. P. Colijn, ${ }^{118}$ J. Collot,${ }^{56}$ P. Conde Muiño, ${ }^{136 a, 136 b}$ E. Coniavitis, ${ }^{50}$ S. H. Connell, ${ }^{32 \mathrm{~b}}$ I. A. Connelly, ${ }^{98}$ S. Constantinescu, ${ }^{27 \mathrm{~b}}$ F. Conventi, ${ }^{67,1}$ A. M. Cooper-Sarkar, ${ }^{131}$ F. Cormier, ${ }^{172}$ K. J. R. Cormier, ${ }^{164}$ M. Corradi, ${ }^{70 a, 70 b}$ E. E. Corrigan, ${ }^{94}$ F. Corriveau, ${ }^{101, \mathrm{~m}}$ A. Cortes-Gonzalez, ${ }^{35}$ M. J. Costa, ${ }^{171}$ D. Costanzo, ${ }^{146}$ G. Cottin, ${ }^{31}$ G. Cowan, ${ }^{91}$ B. E. Cox,${ }^{98}$ J. Crane,${ }^{98}$ K. Cranmer, ${ }^{121}$ S. J. Crawley, ${ }^{55}$ R. A. Creager, ${ }^{133}$ G. Cree, ${ }^{33}$ S. Crépé-Renaudin, ${ }^{56}$ F. Crescioli, ${ }^{132}$ M. Cristinziani, ${ }^{24}$ V. Croft,,${ }^{121}$ G. Crosetti, ${ }^{40 b, 40 a}$ A. Cueto, ${ }^{96}$

T. Cuhadar Donszelmann, ${ }^{146}$ A. R. Cukierman, ${ }^{150}$ M. Curatolo, ${ }^{49}$ J. Cúth, ${ }^{97}$ S. Czekierda, ${ }^{82}$ P. Czodrowski, ${ }^{35}$ M. J. Da Cunha Sargedas De Sousa, ${ }^{58,136 \mathrm{~b}}$ C. Da Via,${ }^{98}$ W. Dabrowski, ${ }^{81 \mathrm{a}}$ T. Dado, ${ }^{28 \mathrm{a}, \mathrm{n}}$ S. Dahbi, ${ }^{34 \mathrm{e}}$ T. Dai, ${ }^{103}$ F. Dallaire, ${ }^{107}$ C. Dallapiccola, ${ }^{100}$ M. Dam, ${ }^{39}$ G. D'amen, ${ }^{23 \mathrm{~b}, 23 \mathrm{a}}$ J. Damp, ${ }^{97}$ J. R. Dandoy, ${ }^{133}$ M. F. Daneri, ${ }^{30}$ N. P. Dang, ${ }^{178, f}$ N. D Dann, ${ }^{98}$ M. Danninger, ${ }^{172}$ V. Dao, ${ }^{35}$ G. Darbo, ${ }^{53 b}$ S. Darmora,${ }^{8}$ O. Dartsi, ${ }^{5}$ A. Dattagupta, ${ }^{127}$ T. Daubney, ${ }^{44}$ S. D' Auria, ${ }^{55}$ W. Davey,${ }^{24}$ C. David, ${ }^{44}$ T. Davidek, ${ }^{139}$ D. R. Davis, ${ }^{47}$ E. Dawe, ${ }^{102}$ I. Dawson, ${ }^{146}$ K. De,${ }^{8}$ R. De Asmundis, ${ }^{67 a}$ A. De Benedetti, ${ }^{124}$ S. De Castro, ${ }^{23 b, 23 a}$ S. De Cecco, ${ }^{70 a, 70 b}$ N. De Groot, ${ }^{117}$ P. de Jong, ${ }^{118}$ H. De la Torre, ${ }^{104}$ F. De Lorenzi, ${ }^{76}$ A. De Maria,,${ }^{51, o}$ D. De Pedis, ${ }^{70 a}$ A. De Salvo, ${ }^{70 a}$ U. De Sanctis, ${ }^{71 a, 71 b}$ A. De Santo,${ }^{153}$ K. De Vasconcelos Corga, ${ }^{99}$ J. B. De Vivie De Regie, ${ }^{128}$ C. Debenedetti, ${ }^{143}$ D. V. Dedovich, ${ }^{77}$ N. Dehghanian, ${ }^{3}$ M. Del Gaudio, ${ }^{40 b, 40 a}$ J. Del Peso, ${ }^{96}$ D. Delgove,${ }^{128}$ F. Deliot,${ }^{142}$ C. M. Delitzsch, ${ }^{7}$ M. Della Pietra ${ }^{67 a, 67 b}$ D. Della Volpe ${ }^{52}$ A. Dell'Acqua, ${ }^{35}$ L. Dell'Asta, ${ }^{25}$ M. Delmastro, ${ }^{5}$ C. Delporte, ${ }^{128}$ P. A. Delsart, ${ }^{56}$ D. A. DeMarco, ${ }^{164}$ S. Demers, ${ }^{180}$ M. Demichev,${ }^{77}$ S. P. Denisov, ${ }^{140}$ D. Denysiuk, ${ }^{118}$ L. D'Eramo, ${ }^{132}$ D. Derendarz, ${ }^{82}$ J. E. Derkaoui, ${ }^{34 d}$ F. Derue, ${ }^{132}$ P. Dervan,${ }^{88}$ K. Desch, ${ }^{24}$ C. Deterre, ${ }^{44}$ K. Dette, ${ }^{164}$ M. R. Devesa, ${ }^{30}$ P. O. Deviveiros ${ }^{35}$ A. Dewhurst ${ }^{141}$ S. Dhaliwal, ${ }^{26}$ F. A. Di Bello, ${ }^{52}$ A. Di Ciaccio, ${ }^{71 a, 71 b}$ L. Di Ciaccio, ${ }^{5}$

W. K. Di Clemente, ${ }^{133}$ C. Di Donato, ${ }^{67 a, 67 b}$ A. Di Girolamo, ${ }^{35}$ B. Di Micco,${ }^{72 a, 72 b}$ R. Di Nardo, ${ }^{35}$ K. F. Di Petrillo, ${ }^{57}$ A. Di Simone, ${ }^{50}$ R. Di Sipio, ${ }^{164}$ D. Di Valentino, ${ }^{33}$ C. Diaconu, ${ }^{99}$ M. Diamond, ${ }^{164}$ F. A. Dias, ${ }^{39}$ T. Dias Do Vale, ${ }^{136 a}$ 
M. A. Diaz, ${ }^{144 a}$ J. Dickinson, ${ }^{18}$ E. B. Diehl,${ }^{103}$ J. Dietrich, ${ }^{19}$ S. Díez Cornell ${ }^{44}$ A. Dimitrievska, ${ }^{18}$ J. Dingfelder, ${ }^{24}$ F. Dittus, ${ }^{35}$ F. Djama, ${ }^{99}$ T. Djobava, ${ }^{156 b}$ J. I. Djuvsland ${ }^{59 a}$ M. A. B. Do Vale, ${ }^{78 c}$ M. Dobre, ${ }^{27 b}$ D. Dodsworth, ${ }^{26}$ C. Doglioni, ${ }^{94}$ J. Dolejsi, ${ }^{139}$ Z. Dolezal, ${ }^{139}$ M. Donadelli, ${ }^{78 d}$ J. Donini,${ }^{37}$ A. D'onofrio, ${ }^{90}$ M. D'Onofrio, ${ }^{88}$ J. Dopke, ${ }^{141}$ A. Doria,${ }^{67 a}$ M. T. Dova, ${ }^{86}$ A. T. Doyle, ${ }^{55}$ E. Drechsler, ${ }^{51}$ E. Dreyer, ${ }^{149}$ T. Dreyer, ${ }^{51}$ M. Dris,${ }^{10}$ Y. Du, ${ }^{58 \mathrm{~b}}$ J. Duarte-Campderros,${ }^{158}$ F. Dubinin, ${ }^{108}$ M. Dubovsky, ${ }^{28 a}$ A. Dubreuil,${ }^{52}$ E. Duchovni, ${ }^{177}$ G. Duckeck, ${ }^{112}$ A. Ducourthial, ${ }^{132}$ O. A. Ducu, ${ }^{107, p}$ D. Duda, ${ }^{113}$ A. Dudarev, ${ }^{35}$ A. C. Dudder ${ }^{97}$ E. M. Duffield, ${ }^{18}$ L. Duflot, ${ }^{128}$ M. Dührssen, ${ }^{35}$ C. Dülsen, ${ }^{179}$ M. Dumancic, ${ }^{177}$ A. E. Dumitriu, ${ }^{27 \mathrm{~b}, \mathrm{q}}$ A. K. Duncan, ${ }^{55}$ M. Dunford, ${ }^{59 \mathrm{a}}$ A. Duperrin, ${ }^{99}$ H. Duran Yildiz, ${ }^{4 \mathrm{a}}$ M. Düren, ${ }^{54}$ A. Durglishvili, ${ }^{156 \mathrm{~b}}$ D. Duschinger, ${ }^{46}$ B. Dutta, ${ }^{44}$ D. Duvnjak, ${ }^{1}$ M. Dyndal, ${ }^{44}$ S. Dysch, ${ }^{98}$ B. S. Dziedzic, ${ }^{82}$ C. Eckardt, ${ }^{44}$ K. M. Ecker, ${ }^{113}$ R. C. Edgar, ${ }^{103}$ T. Eifert, ${ }^{35}$ G. Eigen, ${ }^{17}$ K. Einsweiler, ${ }^{18}$ T. Ekelof,${ }^{169}$ M. El Kacimi, ${ }^{34 \mathrm{c}}$ R. El Kosseifi, ${ }^{99}$ V. Ellajosyula, ${ }^{99}$ M. Ellert, ${ }^{169}$ F. Ellinghaus, ${ }^{179}$ A. A. Elliot,${ }^{90}$ N. Ellis, ${ }^{35}$ J. Elmsheuser, ${ }^{29}$ M. Elsing, ${ }^{35}$ D. Emeliyanov, ${ }^{141}$ Y. Enari, ${ }^{160}$ J. S. Ennis, ${ }^{175}$ M. B. Epland, ${ }^{47}$ J. Erdmann, ${ }^{45}$ A. Ereditato, ${ }^{20}$ S. Errede, ${ }^{170}$ M. Escalier, ${ }^{128}$ C. Escobar ${ }^{171}$ B. Esposito, ${ }^{49}$ O. Estrada Pastor, ${ }^{171}$ A. I. Etienvre, ${ }^{142}$ E. Etzion, ${ }^{158}$ H. Evans, ${ }^{63}$ A. Ezhilov, ${ }^{134}$ M. Ezzi,${ }^{34 \mathrm{e}}$ F. Fabbri, ${ }^{55}$ L. Fabbri, ${ }^{23 b, 23 a}$ V. Fabiani, ${ }^{117}$ G. Facini, ${ }^{92}$ R. M. Faisca Rodrigues Pereira, ${ }^{136 a}$ R. M. Fakhrutdinov, ${ }^{140}$ S. Falciano, ${ }^{70 a}$ P. J. Falke, ${ }^{5}$ S. Falke, ${ }^{5}$ J. Faltova, ${ }^{139}$ Y. Fang, ${ }^{15 \mathrm{a}}$ M. Fanti, ${ }^{66,66 \mathrm{~b}}$ A. Farbin, ${ }^{8}$ A. Farilla, ${ }^{72 \mathrm{a}}$ E. M. Farina, ${ }^{68 \mathrm{a}, 68 \mathrm{~b}}$ T. Farooque,${ }^{104}$ S. Farrell, ${ }^{18}$ S. M. Farrington, ${ }^{175}$ P. Farthouat, ${ }^{35}$ F. Fassi, ${ }^{34 \mathrm{e}}$ P. Fassnacht,${ }^{35}$ D. Fassouliotis, ${ }^{9}$ M. Faucci Giannelli, ${ }^{48}$ A. Favareto, ${ }^{53 b, 53 a}$ W. J. Fawcett, ${ }^{52}$ L. Fayard, ${ }^{128}$ O. L. Fedin, ${ }^{134, \mathrm{r}}$ W. Fedorko, ${ }^{172}$ M. Feickert, ${ }^{41}$ S. Feigl, ${ }^{130}$ L. Feligioni, ${ }^{99}$ C. Feng, ${ }^{58 b}$ E. J. Feng, ${ }^{35}$ M. Feng, ${ }^{47}$ M. J. Fenton, ${ }^{55}$ A. B. Fenyuk, ${ }^{140}$ L. Feremenga,${ }^{8}$ J. Ferrando, ${ }^{44}$ A. Ferrari, ${ }^{169}$ P. Ferrari, ${ }^{118}$ R. Ferrari, ${ }^{68 \mathrm{a}}$ D. E. Ferreira de Lima, ${ }^{59 \mathrm{~b}}$ A. Ferrer, ${ }^{171}$ D. Ferrere, ${ }^{52}$ C. Ferretti, ${ }^{103}$ F. Fiedler, ${ }^{97}$ A. Filipčič,${ }^{89}$ F. Filthaut, ${ }^{117}$ K. D. Finelli, ${ }^{25}$ M. C. N. Fiolhais, ${ }^{136 a, 136 c, s}$ L. Fiorini, ${ }^{171}$ C. Fischer, ${ }^{14}$ W. C. Fisher,${ }^{104}$ N. Flaschel,${ }^{44}$ I. Fleck, ${ }^{148}$ P. Fleischmann, ${ }^{103}$ R. R. M. Fletcher, ${ }^{133}$ T. Flick, ${ }^{179}$ B. M. Flierl, ${ }^{12}$ L. M. Flores, ${ }^{133}$ L. R. Flores Castillo, ${ }^{61 a}$ N. Fomin,${ }^{17}$ G. T. Forcolin, ${ }^{98}$ A. Formica, ${ }^{142}$ F. A. Förster, ${ }^{14}$ A. C. Forti, ${ }^{98}$ A. G. Foster, ${ }^{21}$ D. Fournier, ${ }^{128}$ H. Fox,${ }^{87}$ S. Fracchia, ${ }^{146}$ P. Francavilla, ${ }^{69 a, 69 b}$ M. Franchini, ${ }^{23 b, 23 a}$ S. Franchino, ${ }^{59 a}$ D. Francis,${ }^{35}$ L. Franconi, ${ }^{130}$ M. Franklin, ${ }^{57}$ M. Frate, ${ }^{168}$ M. Fraternali, ${ }^{68 a, 68 b}$ D. Freeborn, ${ }^{92}$ S. M. Fressard-Batraneanu, ${ }^{35}$ B. Freund, ${ }^{107}$ W. S. Freund, ${ }^{78 b}$ D. Froidevaux, ${ }^{35}$ J. A. Frost, ${ }^{131}$ C. Fukunaga, ${ }^{161}$ T. Fusayasu, ${ }^{114}$ J. Fuster, ${ }^{171}$ O. Gabizon, ${ }^{157}$ A. Gabrielli, ${ }^{23 b, 23 a}$ A. Gabrielli, ${ }^{18}$ G. P. Gach, ${ }^{81 a}$ S. Gadatsch, ${ }^{52}$ P. Gadow, ${ }^{113}$ G. Gagliardi, ${ }^{53 b, 53 a}$ L. G. Gagnon, ${ }^{107}$ C. Galea ${ }^{27 b}$ B. Galhardo, ${ }^{136 a, 136 \mathrm{c}}$ E. J. Gallas, ${ }^{131}$ B. J. Gallop, ${ }^{141}$ P. Gallus, ${ }^{138}$ G. Galster, ${ }^{39}$ R. Gamboa Goni, ${ }^{90}$ K. K. Gan, ${ }^{122}$ S. Ganguly, ${ }^{177}$ Y. Gao,${ }^{88}$ Y. S. Gao, ${ }^{150, g}$ C. García, ${ }^{171}$ J. E. García Navarro, ${ }^{171}$ J. A. García Pascual, ${ }^{15 a}$ M. Garcia-Sciveres,${ }^{18}$ R. W. Gardner, ${ }^{36}$ N. Garelli, ${ }^{150}$ V. Garonne, ${ }^{130}$ K. Gasnikova, ${ }^{44}$ A. Gaudiello ${ }^{53 b, 53 a}$ G. Gaudio, ${ }^{68 a}$ I. L. Gavrilenko, ${ }^{108}$ A. Gavrilyuk, ${ }^{109}$ C. Gay, ${ }^{172}$ G. Gaycken, ${ }^{24}$ E. N. Gazis, ${ }^{10}$ C. N. P. Gee,${ }^{141}$ J. Geisen,${ }^{51}$ M. Geisen, ${ }^{97}$ M. P. Geisler, ${ }^{59 a}$ K. Gellerstedt, ${ }^{43 a, 43 b}$ C. Gemme,${ }^{53 b}$ M. H. Genest ${ }^{56}$ C. Geng, ${ }^{103}$ S. Gentile, ${ }^{70 a, 70 b}$ C. Gentsos, ${ }^{159}$ S. George, ${ }^{91}$ D. Gerbaudo, ${ }^{14}$ G. Gessner, ${ }^{45}$ S. Ghasemi ${ }^{148}$ M. Ghasemi Bostanabad, ${ }^{173}$ M. Ghneimat, ${ }^{24}$ B. Giacobbe ${ }^{23 b}$ S. Giagu, ${ }^{70 a}, 70 \mathrm{~b}$ N. Giangiacomi, ${ }^{23 b, 23 a}$ P. Giannetti, ${ }^{69 a}$ S. M. Gibson, ${ }^{91}$ M. Gignac, ${ }^{143}$ D. Gillberg, ${ }^{33}$ G. Gilles, ${ }^{179}$ D. M. Gingrich,${ }^{3, \mathrm{e}}$ M. P. Giordani, ${ }^{64 a, 64 c}$ F. M. Giorgi, ${ }^{23 b}$ P. F. Giraud,${ }^{142}$ P. Giromini,${ }^{57}$ G. Giugliarelli, ${ }^{64 a, 64 c}$ D. Giugni, ${ }^{66 a}$ F. Giuli, ${ }^{131}$ M. Giulini, ${ }^{59 b}$ S. Gkaitatzis, ${ }^{159}$ I. Gkialas,,${ }^{9, t}$ E. L. Gkougkousis, ${ }^{14}$ P. Gkountoumis, ${ }^{10}$ L. K. Gladilin, ${ }^{11}$ C. Glasman, ${ }^{96}$ J. Glatzer, ${ }^{14}$ P. C. F. Glaysher, ${ }^{44}$ A. Glazov, ${ }^{44}$ M. Goblirsch-Kolb, ${ }^{26}$ J. Godlewski, ${ }^{82}$ S. Goldfarb, ${ }^{102}$ T. Golling, ${ }^{52}$ D. Golubkov, ${ }^{140}$ A. Gomes, ${ }^{136 a, 136 b, 136 d}$ R. Goncalves Gama, ${ }^{78}$ R. Gonçalo, ${ }^{136 a}$ G. Gonella, ${ }^{50}$ L. Gonella, ${ }^{21}$ A. Gongadze, ${ }^{77}$ F. Gonnella, ${ }^{21}$ J. L. Gonski, ${ }^{57}$ S. González de la Hoz, ${ }^{171}$ S. Gonzalez-Sevilla, ${ }^{52}$ L. Goossens,${ }^{35}$ P. A. Gorbounov, ${ }^{109}$ H. A. Gordon, ${ }^{29}$ B. Gorini ${ }^{35}$ E. Gorini, ${ }^{65 a, 65 b}$ A. Gorišek, ${ }^{89}$ A. T. Goshaw, ${ }^{47}$ C. Gössling ${ }^{45}$ M. I. Gostkin, ${ }^{77}$ C. A. Gottardo, ${ }^{24}$ C. R. Goudet, ${ }^{128}$ D. Goujdami, ${ }^{34 c}$ A. G. Goussiou, ${ }^{145}$ N. Govender, ${ }^{32 b, u}$ C. Goy, ${ }^{5}$ E. Gozani, ${ }^{157}$ I. Grabowska-Bold, ${ }^{81 a}$ P. O. J. Gradin, ${ }^{169}$ E. C. Graham, ${ }^{88}$ J. Gramling, ${ }^{168}$ E. Gramstad ${ }^{130}$ S. Grancagnolo,${ }^{19}$ V. Gratchev,${ }^{134}$ P. M. Gravila, ${ }^{27 f}$ C. Gray, ${ }^{55}$ H. M. Gray, ${ }^{18}$ Z. D. Greenwood, ${ }^{93, v}$ C. Grefe, ${ }^{24}$ K. Gregersen, ${ }^{92}$ I. M. Gregor, ${ }^{44}$ P. Grenier, ${ }^{150}$ K. Grevtsov, ${ }^{44}$ J. Griffiths, ${ }^{8}$ A. A. Grillo, ${ }^{143}$ K. Grimm, ${ }^{150}$ S. Grinstein, ${ }^{14, w}$ Ph. Gris, ${ }^{37}$ J.-F. Grivaz, ${ }^{128}$ S. Groh, ${ }^{97}$ E. Gross, ${ }^{177}$ J. Grosse-Knetter,${ }^{51}$ G. C. Grossi, ${ }^{93}$ Z. J. Grout, ${ }^{92}$ C. Grud ${ }^{103}$ A. Grummer, ${ }^{116}$ L. Guan, ${ }^{103}$ W. Guan, ${ }^{178}$ J. Guenther, ${ }^{35}$ A. Guerguichon, ${ }^{128}$ F. Guescini, ${ }^{165 a}$ D. Guest,${ }^{168}$ R. Gugel,${ }^{50}$ B. Gui, ${ }^{122}$ T. Guillemin, ${ }^{5}$ S. Guindon, ${ }^{35}$ U. Gul, ${ }^{55}$ C. Gumpert, ${ }^{35}$ J. Guo, ${ }^{58 c}$ W. Guo, ${ }^{103}$ Y. Guo, ${ }^{58 a, x}$ Z. Guo, ${ }^{99}$ R. Gupta, ${ }^{41}$ S. Gurbuz, ${ }^{12 c}$ G. Gustavino, ${ }^{124}$ B. J. Gutelman, ${ }^{157}$ P. Gutierrez, ${ }^{124}$ C. Gutschow, ${ }^{92}$ C. Guyot,${ }^{142}$ M. P. Guzik, ${ }^{81 a}$ C. Gwenlan, ${ }^{131}$ C. B. Gwilliam, ${ }^{88}$ A. Haas, ${ }^{121}$ C. Haber, ${ }^{18}$ H. K. Hadavand,${ }^{8}$ N. Haddad,${ }^{34 \mathrm{e}}$ A. Hadef, ${ }^{58 \mathrm{a}}$ S. Hageböck, ${ }^{24}$ M. Hagihara, ${ }^{166}$ H. Hakobyan, ${ }^{181, a}$ M. Haleem, ${ }^{174}$ J. Haley, ${ }^{125}$ G. Halladjian, ${ }^{104}$ G. D. Hallewell, ${ }^{99}$ K. Hamacher, ${ }^{179}$ P. Hamal, ${ }^{126}$ K. Hamano, ${ }^{173}$ A. Hamilton, ${ }^{32 a}$ G. N. Hamity, ${ }^{146}$ K. Han,${ }^{58 a, y}$ L. Han, ${ }^{58 a}$ S. Han, ${ }^{15 d}$ K. Hanagaki, ${ }^{79, z}$ M. Hance, ${ }^{143}$ D. M. Handl, ${ }^{112}$ B. Haney, ${ }^{133}$ R. Hankache, ${ }^{132}$ P. Hanke, ${ }^{59 a}$ E. Hansen, ${ }^{94}$ J. B. Hansen, ${ }^{39}$ 
J. D. Hansen, ${ }^{39}$ M. C. Hansen, ${ }^{24}$ P. H. Hansen, ${ }^{39}$ K. Hara, ${ }^{166}$ A. S. Hard,${ }^{178}$ T. Harenberg, ${ }^{179}$ S. Harkusha, ${ }^{105}$ P. F. Harrison, ${ }^{175}$ N. M. Hartmann, ${ }^{12}$ Y. Hasegawa, ${ }^{117}$ A. Hasib, ${ }^{48}$ S. Hassani, ${ }^{142}$ S. Haug ${ }^{20}$ R. Hauser, ${ }^{104}$ L. Hauswald, ${ }^{46}$ L. B. Havener, ${ }^{38}$ M. Havranek, ${ }^{138}$ C. M. Hawkes, ${ }^{21}$ R. J. Hawkings, ${ }^{35}$ D. Hayden, ${ }^{104}$ C. Hayes, ${ }^{152}$ C. P. Hays,${ }^{131}$ J. M. Hays, ${ }^{90}$

H. S. Hayward, ${ }^{88}$ S. J. Haywood, ${ }^{141}$ M. P. Heath, ${ }^{48}$ V. Hedberg, ${ }^{94}$ L. Heelan, ${ }^{8}$ S. Heer, ${ }^{24}$ K. K. Heidegger, ${ }^{50}$ J. Heilman, ${ }^{33}$ S. Heim, ${ }^{44}$ T. Heim, ${ }^{18}$ B. Heinemann, ${ }^{44, a a}$ J. J. Heinrich, ${ }^{12}$ L. Heinrich, ${ }^{121}$ C. Heinz, ${ }^{54}$ J. Hejbal, ${ }^{137}$ L. Helary, ${ }^{35}$ A. Held, ${ }^{172}$ S. Hellesund, ${ }^{130}$ S. Hellman, ${ }^{43 a, 43 b}$ C. Helsens, ${ }^{35}$ R. C. W. Henderson, ${ }^{87}$ Y. Heng, ${ }^{178}$ S. Henkelmann, ${ }^{172}$

A. M. Henriques Correia, ${ }^{35}$ G. H. Herbert, ${ }^{19}$ H. Herde, ${ }^{26}$ V. Herget, ${ }^{174}$ Y. Hernández Jiménez,${ }^{32 c}$ H. Herr, ${ }^{97}$ G. Herten, ${ }^{50}$ R. Hertenberger, ${ }^{112}$ L. Hervas, ${ }^{35}$ T. C. Herwig, ${ }^{133}$ G. G. Hesketh, ${ }^{92}$ N. P. Hessey, ${ }^{165 a}$ J. W. Hetherly, ${ }^{41}$ S. Higashino, ${ }^{79}$

E. Higón-Rodriguez, ${ }^{171}$ K. Hildebrand, ${ }^{36}$ E. Hill,,${ }^{173}$ J. C. Hill, ${ }^{31}$ K. K. Hill, ${ }^{29}$ K. H. Hiller, ${ }^{44}$ S. J. Hillier, ${ }^{21}$ M. Hils, ${ }^{46}$

I. Hinchliffe, ${ }^{18}$ M. Hirose, ${ }^{129}$ D. Hirschbuehl, ${ }^{179}$ B. Hiti, ${ }^{89}$ O. Hladik, ${ }^{137}$ D. R. Hlaluku, ${ }^{32 c}$ X. Hoad, ${ }^{48}$ J. Hobbs, ${ }^{152}$

N. Hod, ${ }^{165}$ M. C. Hodgkinson, ${ }^{146}$ A. Hoecker, ${ }^{35}$ M. R. Hoeferkamp, ${ }^{116}$ F. Hoenig, ${ }^{112}$ D. Hohn,${ }^{24}$ D. Hohov, ${ }^{128}$

T. R. Holmes, ${ }^{36}$ M. Holzbock, ${ }^{112}$ M. Homann, ${ }^{45}$ S. Honda,${ }^{166}$ T. Honda, ${ }^{79}$ T. M. Hong, ${ }^{135}$ A. Hönle, ${ }^{113}$ B. H. Hooberman, ${ }^{170}$

W. H. Hopkins, ${ }^{127}$ Y. Horii, ${ }^{115}$ P. Horn ${ }^{46}$ A. J. Horton, ${ }^{149}$ L. A. Horyn, ${ }^{36}$ J-Y. Hostachy, ${ }^{56}$ A. Hostiuc, ${ }^{145}$ S. Hou, ${ }^{155}$

A. Hoummada, ${ }^{34 a}$ J. Howarth, ${ }^{98}$ J. Hoya,${ }^{86}$ M. Hrabovsky, ${ }^{126}$ J. Hrdinka, ${ }^{35}$ I. Hristova, ${ }^{19}$ J. Hrivnac, ${ }^{128}$ A. Hrynevich, ${ }^{106}$

T. Hryn'ova, ${ }^{5}$ P. J. Hsu, ${ }^{62}$ S.-C. Hsu, ${ }^{145}$ Q. Hu, ${ }^{29}$ S. Hu,${ }^{58 c}$ Y. Huang, ${ }^{15 a}$ Z. Hubacek, ${ }^{138}$ F. Hubaut, ${ }^{99}$ M. Huebner, ${ }^{24}$

F. Huegging, ${ }^{24}$ T. B. Huffman, ${ }^{131}$ E. W. Hughes, ${ }^{38}$ M. Huhtinen, ${ }^{35}$ R. F. H. Hunter, ${ }^{33}$ P. Huo, ${ }^{152}$ A. M. Hupe, ${ }^{33}$

N. Huseynov, ${ }^{77, \mathrm{~d}}$ J. Huston, ${ }^{104} \mathrm{~J}$. Huth ${ }^{57}$ R. Hyneman, ${ }^{103}$ G. Iacobucci, ${ }^{52}$ G. Iakovidis, ${ }^{29}$ I. Ibragimov, ${ }^{148}$

L. Iconomidou-Fayard, ${ }^{128}$ Z. Idrissi, ${ }^{34}$ P. Iengo, ${ }^{35}$ R. Ignazzi, ${ }^{39}$ O. Igonkina, ${ }^{18, b b}$ R. Iguchi, ${ }^{160}$ T. Iizawa, ${ }^{52}$ Y. Ikegami, ${ }^{79}$ M. Ikeno, ${ }^{79}$ D. Iliadis, ${ }^{159} \mathrm{~N}$. Ilic, ${ }^{150} \mathrm{~F}$. Iltzsche, ${ }^{46} \mathrm{G}$. Introzzi, ${ }^{68 \mathrm{a}, 68 \mathrm{~b}} \mathrm{M}$. Iodice, ${ }^{72 \mathrm{a}} \mathrm{K}$. Iordanidou, ${ }^{38} \mathrm{~V}$. Ippolito, ${ }^{70 \mathrm{a}, 70 \mathrm{~b}}$

M. F. Isacson, ${ }^{169} \mathrm{~N}$. Ishijima, ${ }^{129} \mathrm{M}$. Ishino, ${ }^{160} \mathrm{M}$. Ishitsuka, ${ }^{162} \mathrm{~W}$. Islam, ${ }^{125} \mathrm{C}$. Issever, ${ }^{131} \mathrm{~S}$. Istin, ${ }^{12 c, c c} \mathrm{~F}$. Ito, ${ }^{166}$

J. M. Iturbe Ponce, ${ }^{61 \mathrm{a}}$ R. Iuppa, ${ }^{73 a, 73 b}$ A. Ivina, ${ }^{177}$ H. Iwasaki, ${ }^{79}$ J. M. Izen, ${ }^{42}$ V. Izzo, ${ }^{67 a}$ S. Jabbar, ${ }^{3}$ P. Jacka,,${ }^{137}$ P. Jackson, ${ }^{1}$

R. M. Jacobs, ${ }^{24}$ V. Jain, ${ }^{2}$ G. Jäkel, ${ }^{179}$ K. B. Jakobi, ${ }^{97}$ K. Jakobs, ${ }^{50}$ S. Jakobsen, ${ }^{74}$ T. Jakoubek, ${ }^{137}$ D. O. Jamin, ${ }^{125}$

D. K. Jana, ${ }^{93}$ R. Jansky, ${ }^{52}$ J. Janssen, ${ }^{24}$ M. Janus, ${ }^{51}$ P. A. Janus, ${ }^{81 a}$ G. Jarlskog, ${ }^{94}$ N. Javadov, ${ }^{77, d}$ T. Javůrek, ${ }^{50}$

M. Javurkova ${ }^{50}$ F. Jeanneau, ${ }^{142}$ L. Jeanty,${ }^{18}$ J. Jejelava, ${ }^{156 a, d d}$ A. Jelinskas,${ }^{175}$ P. Jenni, ${ }^{50, e e}$ J. Jeong, ${ }^{44}$ C. Jeske, ${ }^{175}$ S. Jézéquel, ${ }^{5}$ H. Ji, ${ }^{178}$ J. Jia, ${ }^{152}$ H. Jiang,${ }^{76}$ Y. Jiang,${ }^{58 a}$ Z. Jiang, ${ }^{150, f f}$ S. Jiggins,${ }^{50}$ F. A. Jimenez Morales,,${ }^{37}$ J. Jimenez Pena, ${ }^{171}$ S. Jin, ${ }^{15 \mathrm{c}}$ A. Jinaru, ${ }^{27 \mathrm{~b}}$ O. Jinnouchi, ${ }^{162}$ H. Jivan, ${ }^{32 \mathrm{c}}$ P. Johansson, ${ }^{146}$ K. A. Johns, ${ }^{7}$ C. A. Johnson, ${ }^{63}$ W. J. Johnson, ${ }^{145}$

K. Jon-And, ${ }^{43 a, 43 b}$ R. W. L. Jones, ${ }^{87}$ S. D. Jones, ${ }^{153}$ S. Jones, ${ }^{7}$ T. J. Jones, ${ }^{88}$ J. Jongmanns, ${ }^{59 a}$ P. M. Jorge, ${ }^{136 a, 136 b}$ J. Jovicevic, ${ }^{165}$ X. Ju, ${ }^{178}$ J. J. Junggeburth ${ }^{113}$ A. Juste Rozas, ${ }^{14, w}$ A. Kaczmarska, ${ }^{82}$ M. Kado, ${ }^{128}$ H. Kagan ${ }^{122}$ M. Kagan, ${ }^{150}$ T. Kaji, ${ }^{176}$ E. Kajomovitz, ${ }^{157}$ C. W. Kalderon, ${ }^{94}$ A. Kaluza, ${ }^{97}$ S. Kama, ${ }^{41}$ A. Kamenshchikov, ${ }^{140}$ L. Kanjir, ${ }^{89}$ Y. Kano, ${ }^{160}$ V. A. Kantserov, ${ }^{110}$ J. Kanzaki, ${ }^{79}$ B. Kaplan, ${ }^{121}$ L. S. Kaplan, ${ }^{178}$ D. Kar, ${ }^{32 \mathrm{c}}$ M. J. Kareem, ${ }^{165 \mathrm{~b}}$ E. Karentzos ${ }^{10}$ S. N. Karpov, ${ }^{77}$ Z. M. Karpova, ${ }^{77}$ V. Kartvelishvili, ${ }^{87}$ A. N. Karyukhin, ${ }^{140}$ K. Kasahara, ${ }^{166}$ L. Kashif, ${ }^{178}$ R. D. Kass, ${ }^{122}$ A. Kastanas, ${ }^{151}$

Y. Kataoka, ${ }^{160}$ C. Kato, ${ }^{160}$ J. Katzy, ${ }^{44}$ K. Kawade,${ }^{80}$ K. Kawagoe, ${ }^{85}$ T. Kawamoto, ${ }^{160}$ G. Kawamura, ${ }^{51}$ E. F. Kay, ${ }^{88}$

V. F. Kazanin, ${ }^{120 b, 120 a}$ R. Keeler, ${ }^{173}$ R. Kehoe, ${ }^{41}$ J. S. Keller, ${ }^{33}$ E. Kellermann,,${ }^{94}$ J. J. Kempster, ${ }^{21}$ J. Kendrick, ${ }^{21}$ O. Kepka, ${ }^{137}$ S. Kersten, ${ }^{179}$ B. P. Kerševan, ${ }^{89}$ R. A. Keyes, ${ }^{101}$ M. Khader, ${ }^{170}$ F. Khalil-Zada, ${ }^{13}$ A. Khanov, ${ }^{125}$ A. G. Kharlamov, ${ }^{120 b, 120 a}$ T. Kharlamova, ${ }^{120 b, 120 \mathrm{a}}$ A. Khodinov, ${ }^{163}$ T. J. Khoo, ${ }^{52}$ E. Khramov, ${ }^{77}$ J. Khubua, ${ }^{156 b}$ S. Kido, ${ }^{80}$ M. Kiehn, ${ }^{52}$ C. R. Kilby, ${ }^{91}$ S. H. Kim, ${ }^{166}$ Y. K. Kim, ${ }^{36}$ N. Kimura, ${ }^{64 a, 64 c}$ O. M. Kind, ${ }^{19}$ B. T. King, ${ }^{88}$ D. Kirchmeier, ${ }^{46}$ J. Kirk, ${ }^{141}$ A. E. Kiryunin,${ }^{113}$ T. Kishimoto, ${ }^{160}$ D. Kisielewska, ${ }^{81 a}$ V. Kitali, ${ }^{44}$ O. Kivernyk, ${ }^{5}$ E. Kladiva, ${ }^{28 b, a}$ T. Klapdor-Kleingrothaus, ${ }^{50}$ M. H. Klein, ${ }^{103}$

M. Klein, ${ }^{88}$ U. Klein, ${ }^{88}$ K. Kleinknecht, ${ }^{97}$ P. Klimek, ${ }^{119}$ A. Klimentov, ${ }^{29}$ R. Klingenberg, ${ }^{45, a}$ T. Klingl, ${ }^{24}$

T. Klioutchnikova, ${ }^{35}$ F. F. Klitzner, ${ }^{112}$ P. Kluit, ${ }^{118}$ S. Kluth, ${ }^{113}$ E. Kneringer, ${ }^{74}$ E. B. F. G. Knoops, ${ }^{99}$ A. Knue, ${ }^{50}$ A. Kobayashi, ${ }^{160}$ D. Kobayashi, ${ }^{85}$ T. Kobayashi, ${ }^{160}$ M. Kobel,${ }^{46}$ M. Kocian, ${ }^{150}$ P. Kodys, ${ }^{139}$ T. Koffas, ${ }^{33}$ E. Koffeman, ${ }^{118}$ N. M. Köhler, ${ }^{113}$ T. Koi, ${ }^{150}$ M. Kolb, ${ }^{59 b}$ I. Koletsou, ${ }^{5}$ T. Kondo, ${ }^{79}$ N. Kondrashova, ${ }^{58 c}$ K. Köneke, ${ }^{50}$ A. C. König, ${ }^{17}$

T. Kono,${ }^{79}$ R. Konoplich, ${ }^{121, \mathrm{gg}}$ V. Konstantinides, ${ }^{92}$ N. Konstantinidis, ${ }^{92}$ B. Konya ${ }^{94}$ R. Kopeliansky, ${ }^{63}$ S. Koperny, ${ }^{81 a}$

K. Korcyl, ${ }^{82}$ K. Kordas, ${ }^{159}$ A. Korn, ${ }^{92}$ I. Korolkov, ${ }^{14}$ E. V. Korolkova, ${ }^{146}$ O. Kortner, ${ }^{113}$ S. Kortner, ${ }^{113}$ T. Kosek, ${ }^{139}$ V. V. Kostyukhin, ${ }^{24}$ A. Kotwal, ${ }^{47}$ A. Koulouris, ${ }^{10}$ A. Kourkoumeli-Charalampidi, ${ }^{68 a, 68 b}$ C. Kourkoumelis, ${ }^{9}$ E. Kourlitis, ${ }^{146}$ V. Kouskoura, ${ }^{29}$ A. B. Kowalewska, ${ }^{82}$ R. Kowalewski, ${ }^{173}$ T. Z. Kowalski, ${ }^{81 a}$ C. Kozakai, ${ }^{160}$ W. Kozanecki, ${ }^{142}$ A. S. Kozhin, ${ }^{140}$ V. A. Kramarenko, ${ }^{111}$ G. Kramberger ${ }^{89}$ D. Krasnopevtsev, ${ }^{110}$ M. W. Krasny, ${ }^{132}$ A. Krasznahorkay, ${ }^{35}$ D. Krauss, ${ }^{113}$ J. A. Kremer, ${ }^{81 a}$ J. Kretzschmar, ${ }^{88}$ P. Krieger, ${ }^{164}$ K. Krizka, ${ }^{18}$ K. Kroeninger ${ }^{45}$ H. Kroha, ${ }^{113}$ J. Kroll, ${ }^{137}$ J. Kroll, ${ }^{133}$ J. Krstic, ${ }^{16}$ U. Kruchonak,${ }^{77}$ H. Krüger, ${ }^{24}$ N. Krumnack, ${ }^{76}$ M. C. Kruse, ${ }^{47}$ T. Kubota, ${ }^{102}$ S. Kuday, ${ }^{46}$ J. T. Kuechler, ${ }^{179}$ S. Kuehn, ${ }^{35}$ A. Kugel, ${ }^{59 a}$ F. Kuger, ${ }^{174}$ T. Kuhl, ${ }^{44}$ V. Kukhtin, ${ }^{77}$ R. Kukla, ${ }^{99}$ Y. Kulchitsky, ${ }^{105}$ 
S. Kuleshov, ${ }^{144 b}$ Y. P. Kulinich, ${ }^{170}$ M. Kuna, ${ }^{56}$ T. Kunigo, ${ }^{83}$ A. Kupco, ${ }^{137}$ T. Kupfer, ${ }^{45}$ O. Kuprash, ${ }^{158}$ H. Kurashige, ${ }^{80}$ L. L. Kurchaninov, ${ }^{165 a}$ Y. A. Kurochkin, ${ }^{105}$ M. G. Kurth, ${ }^{15 d}$ E. S. Kuwertz, ${ }^{173}$ M. Kuze, ${ }^{162}$ J. Kvita, ${ }^{126}$ T. Kwan, ${ }^{101}$ A. La Rosa, ${ }^{113}$ J. L. La Rosa Navarro, ${ }^{78 d}$ L. La Rotonda, ${ }^{40 b, 40 a}$ F. La Ruffa, ${ }^{40 b, 40 a}$ C. Lacasta, ${ }^{171}$ F. Lacava, ${ }^{70 a, 70 b}$ J. Lacey, ${ }^{44}$ D. P. J. Lack, ${ }^{98}$ H. Lacker, ${ }^{19}$ D. Lacour, ${ }^{132}$ E. Ladygin,${ }^{77}$ R. Lafaye, ${ }^{5}$ B. Laforge, ${ }^{132}$ T. Lagouri, ${ }^{32 \mathrm{c}}$ S. Lai, ${ }^{51}$ S. Lammers, ${ }^{63}$ W. Lampl, ${ }^{7}$ E. Lançon, ${ }^{29}$ U. Landgraf,${ }^{50}$ M. P. J. Landon, ${ }^{90}$ M. C. Lanfermann,${ }^{52}$ V. S. Lang, ${ }^{44}$ J. C. Lange, ${ }^{14}$

R. J. Langenberg, ${ }^{35}$ A. J. Lankford, ${ }^{168}$ F. Lanni, ${ }^{29}$ K. Lantzsch, ${ }^{24}$ A. Lanza, ${ }^{68 a}$ A. Lapertosa, ${ }^{53 b, 53 a}$ S. Laplace, ${ }^{132}$ J. F. Laporte, ${ }^{142}$ T. Lari, ${ }^{66 a}$ F. Lasagni Manghi, ${ }^{23 b, 23 a}$ M. Lassnig, ${ }^{35}$ T. S. Lau, ${ }^{61 a}$ A. Laudrain, ${ }^{128}$ A. T. Law, ${ }^{143}$ P. Laycock, ${ }^{88}$ M. Lazzaroni, ${ }^{66 a, 66 b}$ B. Le, ${ }^{102}$ O. Le Dortz, ${ }^{132}$ E. Le Guirriec, ${ }^{99}$ E. P. Le Quilleuc, ${ }^{142}$ M. LeBlanc, ${ }^{7}$ T. LeCompte, ${ }^{6}$ F. Ledroit-Guillon, ${ }^{56}$ C. A. Lee, ${ }^{29}$ G. R. Lee, ${ }^{144 a}$ L. Lee,${ }^{57}$ S. C. Lee, ${ }^{155}$ B. Lefebvre, ${ }^{101}$ M. Lefebvre, ${ }^{173}$ F. Legger, ${ }^{112}$ C. Leggett, ${ }^{18}$ N. Lehmann, ${ }^{179}$ G. Lehmann Miotto, ${ }^{35}$ W. A. Leight, ${ }^{44}$ A. Leisos, ${ }^{159, \text { hh }}$ M. A. L. Leite, ${ }^{78 d}$ R. Leitner, ${ }^{139}$ D. Lellouch ${ }^{177}$ B. Lemmer, ${ }^{51}$ K. J. C. Leney, ${ }^{92}$ T. Lenz,${ }^{24}$ B. Lenzi, ${ }^{35}$ R. Leone, ${ }^{7}$ S. Leone, ${ }^{69 a}$ C. Leonidopoulos,${ }^{48}$ G. Lerner ${ }^{153}$ C. Leroy, ${ }^{107}$ R. Les, ${ }^{164}$ A. A. J. Lesage, ${ }^{142}$ C. G. Lester,${ }^{31}$ M. Levchenko, ${ }^{134}$ J. Levêque, ${ }^{5}$ D. Levin, ${ }^{103}$ L. J. Levinson, ${ }^{177}$ D. Lewis, ${ }^{90}$ B. Li ${ }^{103}$ C-Q. Li ${ }^{58 \mathrm{a}} \mathrm{H} . \mathrm{Li},{ }^{58 \mathrm{~b}} \mathrm{~L} . \mathrm{Li},{ }^{58 \mathrm{c}} \mathrm{Q} . \mathrm{Li},{ }^{15 \mathrm{~d}} \mathrm{Q} . \mathrm{Y} . \mathrm{Li},{ }^{58 \mathrm{a}} \mathrm{S} . \mathrm{Li},{ }^{58 \mathrm{~d}, 58 \mathrm{c}} \mathrm{X} . \mathrm{Li},{ }^{58 \mathrm{c}} \mathrm{Y}^{\mathrm{Li}},{ }^{148}$ Z. Liang, ${ }^{15 a}$ B. Liberti, ${ }^{71 a}$ A. Liblong, ${ }^{164}$ K. Lie, ${ }^{61 \mathrm{c}} \mathrm{S}$. Liem, ${ }^{118}$ A. Limosani, ${ }^{154}$ C. Y. Lin, ${ }^{31}$ K. Lin, ${ }^{104}$ T. H. Lin, ${ }^{97}$ R. A. Linck, ${ }^{63}$ B. E. Lindquist, ${ }^{152}$ A. L. Lionti, ${ }^{52}$ E. Lipeles, ${ }^{133}$ A. Lipniacka, ${ }^{17}$ M. Lisovyi, ${ }^{59 b}$ T. M. Liss, ${ }^{170, i i}$ A. Lister, ${ }^{172}$ A. M. Litke, ${ }^{143}$ J. D. Little, ${ }^{8}$ B. Liu, ${ }^{76}$ B. L Liu, ${ }^{6}$ H. B. Liu, ${ }^{29}$ H. Liu, ${ }^{103}$ J. B. Liu, ${ }^{58 a}$ J. K. K. Liu, ${ }^{131}$ K. Liu, ${ }^{132}$ M. Liu, ${ }^{58 a}$ P. Liu, ${ }^{18}$ Y. Liu, ${ }^{15 a}$ Y. L. Liu, ${ }^{58 \mathrm{a}}$ Y. W. Liu, ${ }^{58 \mathrm{a}}$ M. Livan, ${ }^{6 \mathrm{a}, 68 \mathrm{~b}}$ A. Lleres, ${ }^{56}$ J. Llorente Merino, ${ }^{15 \mathrm{a}}$ S. L. Lloyd, ${ }^{90}$ C. Y. Lo, ${ }^{61 \mathrm{~b}}$ F. Lo Sterzo, ${ }^{41}$ E. M. Lobodzinska, ${ }^{44}$ P. Loch, ${ }^{7}$ F. K. Loebinger, ${ }^{98}$ K. M. Loew,${ }^{26}$ T. Lohse, ${ }^{19}$ K. Lohwasser, ${ }^{146}$ M. Lokajicek, ${ }^{137}$ B. A. Long, ${ }^{25}$ J. D. Long, ${ }^{170}$ R. E. Long, ${ }^{87}$ L. Longo, ${ }^{65 a, 65 b}$ K. A. Looper, ${ }^{122}$ J. A. Lopez, ${ }^{144 b}$ I. Lopez Paz, ${ }^{14}$ A. Lopez Solis, ${ }^{146}$ J. Lorenz, ${ }^{112}$ N. Lorenzo Martinez, ${ }^{5}$ M. Losada, ${ }^{22}$ P. J. Lösel,${ }^{12}$ A. Lösle, ${ }^{50}$ X. Lou, ${ }^{44}$ X. Lou, ${ }^{15 a}$ A. Lounis, ${ }^{128}$ J. Love, ${ }^{6}$ P. A. Love, ${ }^{87}$ J. J. Lozano Bahilo, ${ }^{171}$ H. Lu ${ }^{61 a}$ M. Lu, ${ }^{58 a}$ N. Lu, ${ }^{103}$ Y. J. Lu, ${ }^{62}$ H. J. Lubatti, ${ }^{145}$ C. Luci ${ }^{70 a, 70 b}$ A. Lucotte,${ }^{56}$ C. Luedtke,${ }^{50}$ F. Luehring, ${ }^{63}$ I. Luise, ${ }^{132}$ W. Lukas, ${ }^{74}$ L. Luminari, ${ }^{70 a}$ B. Lund-Jensen,${ }^{151}$ M. S. Lutz, ${ }^{100}$ P. M. Luzi, ${ }^{132}$ D. Lynn, ${ }^{29}$ R. Lysak, ${ }^{137}$ E. Lytken, ${ }^{94}$ F. Lyu, ${ }^{15 a}$ V. Lyubushkin, ${ }^{77}$ H. Ma,${ }^{29}$ L. L. Ma ${ }^{58 b}$ Y. Ma,${ }^{58 b}$ G. Maccarrone, ${ }^{49}$ A. Macchiolo, ${ }^{113}$ C. M. Macdonald, ${ }^{146}$ J. Machado Miguens, ${ }^{133,136 \mathrm{~b}}$ D. Madaffari, ${ }^{171}$ R. Madar, ${ }^{37}$ W. F. Mader, ${ }^{46}$ A. Madsen, ${ }^{44}$ N. Madysa, ${ }^{46}$ J. Maeda, ${ }^{80}$ K. Maekawa, ${ }^{160}$ S. Maeland, ${ }^{17}$ T. Maeno, ${ }^{29}$ A. S. Maevskiy, ${ }^{111}$ V. Magerl,${ }^{50}$ C. Maidantchik, ${ }^{78 b}$ T. Maier, ${ }^{112}$ A. Maio, ${ }^{136 a, 136 b, 136 \mathrm{~d}}$ O. Majersky, ${ }^{28 \mathrm{a}}$ S. Majewski, ${ }^{127}$ Y. Makida, ${ }^{79}$ N. Makovec, ${ }^{128}$ B. Malaescu, ${ }^{132}$ Pa. Malecki, ${ }^{82}$ V. P. Maleev, ${ }^{134}$ F. Malek,${ }^{56}$ U. Mallik,${ }^{75}$ D. Malon, ${ }^{6}$ C. Malone, ${ }^{31}$ S. Maltezos, ${ }^{10}$ S. Malyukov,${ }^{35}$ J. Mamuzic, ${ }^{171}$ G. Mancini, ${ }^{49}$ I. Mandić ${ }^{89}$ J. Maneira, ${ }^{136 a}$ L. Manhaes de Andrade Filho, ${ }^{78 a}$ J. Manjarres Ramos, ${ }^{46}$ K. H. Mankinen, ${ }^{94}$ A. Mann, ${ }^{112}$ A. Manousos,${ }^{74}$ B. Mansoulie, ${ }^{142}$ J. D. Mansour, ${ }^{15 a}$ M. Mantoani, ${ }^{51}$ S. Manzoni, ${ }^{66,66 b}$ G. Marceca,${ }^{30}$ L. March, ${ }^{52}$ L. Marchese, ${ }^{131}$ G. Marchiori, ${ }^{132}$ M. Marcisovsky, ${ }^{137}$ C. A. Marin Tobon, ${ }^{35}$ M. Marjanovic, ${ }^{37}$ D. E. Marley, ${ }^{103}$ F. Marroquim, ${ }^{78 b}$ Z. Marshall, ${ }^{18}$ M. U. F Martensson, ${ }^{169}$ S. Marti-Garcia, ${ }^{171}$ C. B. Martin, ${ }^{122}$ T. A. Martin, ${ }^{175}$ V. J. Martin, ${ }^{48}$ B. Martin dit Latour, ${ }^{17}$ M. Martinez, ${ }^{14, w}$ V. I. Martinez Outschoorn, ${ }^{100}$ S. Martin-Haugh, ${ }^{141}$ V. S. Martoiu, ${ }^{27 b}$ A. C. Martyniuk, ${ }^{92}$ A. Marzin, ${ }^{35}$ L. Masetti, ${ }^{97}$ T. Mashimo, ${ }^{160}$ R. Mashinistov, ${ }^{108}$ J. Masik, ${ }^{98}$ A. L. Maslennikov, ${ }^{120 b, 120 \mathrm{a}}$ L. H. Mason, ${ }^{102}$ L. Massa,${ }^{71 a, 71 \mathrm{~b}}$ P. Mastrandrea, ${ }^{5}$ A. Mastroberardino, ${ }^{40 \mathrm{~b}, 40 \mathrm{a}}$ T. Masubuchi, ${ }^{160}$ P. Mättig, ${ }^{179}$ J. Maurer, ${ }^{27 b}$ B. Maček, ${ }^{89}$ S. J. Maxfield, ${ }^{88}$ D. A. Maximov, ${ }^{120 b, 120 a}$ R. Mazini,${ }^{155}$ I. Maznas, ${ }^{159}$

S. M. Mazza, ${ }^{143}$ N. C. Mc Fadden, ${ }^{116}$ G. Mc Goldrick, ${ }^{164}$ S. P. Mc Kee, ${ }^{103}$ A. McCarn, ${ }^{103}$ T. G. McCarthy, ${ }^{113}$

L. I. McClymont ${ }^{92}$ E. F. McDonald, ${ }^{102}$ J. A. Mcfayden, ${ }^{35}$ G. Mchedlidze,${ }^{51}$ M. A. McKay, ${ }^{41}$ K. D. McLean,${ }^{173}$ S. J. McMahon, ${ }^{141}$ P. C. McNamara ${ }^{102}$ C. J. McNicol, ${ }^{175}$ R. A. McPherson, ${ }^{173, m}$ J. E. Mdhluli ${ }^{32 c}$ Z. A. Meadows, ${ }^{100}$ S. Meehan ${ }^{145}$ T. M. Megy, ${ }^{50}$ S. Mehlhase, ${ }^{112}$ A. Mehta, ${ }^{88}$ T. Meideck,${ }^{56}$ B. Meirose,${ }^{42}$ D. Melini,${ }^{171, j j}$ B. R. Mellado Garcia, ${ }^{32 c}$ J. D. Mellenthin,${ }^{51}$ M. Melo, ${ }^{28 a}$ F. Meloni,${ }^{20}$ A. Melzer, ${ }^{24}$ S. B. Menary, ${ }^{98}$

E. D. Mendes Gouveia, ${ }^{136 a}$ L. Meng, ${ }^{88}$ X. T. Meng, ${ }^{103}$ A. Mengarelli, ${ }^{23 b, 23 a}$ S. Menke, ${ }^{113}$ E. Meoni, ${ }^{40 b, 40 a}$ S. Mergelmeyer,${ }^{19}$ C. Merlassino, ${ }^{20}$ P. Mermod ${ }^{52}$ L. Merola,${ }^{67 a, 67 b}$ C. Meroni, ${ }^{66 a}$ F. S. Merritt, ${ }^{36}$ A. Messina, ${ }^{70 a, 70 b}$ J. Metcalfe, ${ }^{6}$ A. S. Mete, ${ }^{168}$ C. Meyer, ${ }^{133}$ J. Meyer, ${ }^{157}$ J-P. Meyer, ${ }^{142}$ H. Meyer Zu Theenhausen, ${ }^{59 a}$ F. Miano, ${ }^{153}$ R. P. Middleton, ${ }^{141}$ L. Mijović, ${ }^{48}$ G. Mikenberg, ${ }^{177}$ M. Mikestikova, ${ }^{137}$ M. Mikuž, ${ }^{89}$ M. Milesi, ${ }^{102}$ A. Milic, ${ }^{164}$ D. A. Millar, ${ }^{90}$ D. W. Miller, ${ }^{36}$ A. Milov, ${ }^{177}$ D. A. Milstead, ${ }^{43 a, 43 b}$ A. A. Minaenko, ${ }^{140}$ M. Miñano Moya, ${ }^{171}$ I. A. Minashvili, ${ }^{156 b}$ A. I. Mincer, ${ }^{121}$ B. Mindur, ${ }^{81 a}$ M. Mineev ${ }^{77}$ Y. Minegishi, ${ }^{160}$ Y. Ming, ${ }^{178}$ L. M. Mir, ${ }^{14}$ A. Mirto, ${ }^{65 a, 65 b}$ K. P. Mistry, ${ }^{133}$ T. Mitani, ${ }^{176}$ J. Mitrevski, ${ }^{112}$ V. A. Mitsou, ${ }^{171}$ A. Miucci, ${ }^{20}$ P. S. Miyagawa, ${ }^{146}$ A. Mizukami, ${ }^{79}$ J. U. Mjörnmark, ${ }^{94}$ T. Mkrtchyan, ${ }^{181}$ M. Mlynarikova, ${ }^{139}$ T. Moa, ${ }^{43 a, 43 b}$ K. Mochizuki, ${ }^{107}$ P. Mogg, ${ }^{50}$ S. Mohapatra, ${ }^{38}$ S. Molander, ${ }^{43 a, 43 b}$ R. Moles-Valls,${ }^{24}$ M. C. Mondragon, ${ }^{104}$ K. Mönig, ${ }^{44}$ J. Monk, ${ }^{39}$ E. Monnier, ${ }^{99}$ A. Montalbano, ${ }^{149}$ J. Montejo Berlingen, ${ }^{35}$ F. Monticelli, ${ }^{86}$ S. Monzani, ${ }^{66 a}$ 
R. W. Moore,${ }^{3}$ N. Morange, ${ }^{128}$ D. Moreno, ${ }^{22}$ M. Moreno Llácer,${ }^{35}$ P. Morettini, ${ }^{53 b}$ M. Morgenstern, ${ }^{118}$ S. Morgenstern,${ }^{35}$ D. Mori, ${ }^{149}$ T. Mori, ${ }^{160}$ M. Morii ${ }^{57}$ M. Morinaga, ${ }^{176}$ V. Morisbak, ${ }^{130}$ A. K. Morley, ${ }^{35}$ G. Mornacchi, ${ }^{35}$ A. P. Morris ${ }^{92}$ J. D. Morris, ${ }^{90}$ L. Morvaj, ${ }^{152}$ P. Moschovakos, ${ }^{10}$ M. Mosidze, ${ }^{156 b}$ H. J. Moss, ${ }^{146}$ J. Moss, ${ }^{150, k k}$ K. Motohashi, ${ }^{162}$ R. Mount, ${ }^{150}$ E. Mountricha, ${ }^{35}$ E. J. W. Moyse, ${ }^{100}$ S. Muanza, ${ }^{99}$ F. Mueller, ${ }^{113}$ J. Mueller, ${ }^{135}$ R. S. P. Mueller, ${ }^{12}$ D. Muenstermann, ${ }^{87}$ P. Mullen, ${ }^{55}$ G. A. Mullier, ${ }^{20}$ F. J. Munoz Sanchez ${ }^{98}$ P. Murin, ${ }^{28 b}$ W. J. Murray, ${ }^{175,141}$ A. Murrone,${ }^{66 a, 66 b}$ M. Muškinja ${ }^{89}$ C. Mwewa, ${ }^{32 a}$ A. G. Myagkov, ${ }^{140,11}$ J. Myers, ${ }^{127}$ M. Myska, ${ }^{138}$ B. P. Nachman, ${ }^{18}$ O. Nackenhorst, ${ }^{45}$ K. Nagai, ${ }^{131}$ K. Nagano, ${ }^{79}$ Y. Nagasaka,${ }^{60}$ K. Nagata, ${ }^{166}$ M. Nagel, ${ }^{50}$ E. Nagy, ${ }^{99}$ A. M. Nairz,${ }^{35}$ Y. Nakahama,${ }^{115}$ K. Nakamura, ${ }^{79}$ T. Nakamura, ${ }^{160}$ I. Nakano, ${ }^{123}$ H. Nanjo, ${ }^{129}$ F. Napolitano, ${ }^{59 a}$ R. F. Naranjo Garcia, ${ }^{44}$ R. Narayan, ${ }^{11}$ D. I. Narrias Villar, ${ }^{59 a}$ I. Naryshkin, ${ }^{134}$ T. Naumann, ${ }^{44}$ G. Navarro, ${ }^{22}$ R. Nayyar, ${ }^{7}$ H. A. Neal, ${ }^{103}$ P. Y. Nechaeva, ${ }^{108}$ T. J. Neep,${ }^{142}$ A. Negri, ${ }^{68 a, 68 b}$ M. Negrini, ${ }^{23 b}$ S. Nektarijevic, ${ }^{117}$ C. Nellist, ${ }^{51}$ M. E. Nelson, ${ }^{131}$ S. Nemecek, ${ }^{137}$ P. Nemethy, ${ }^{121}$ M. Nessi, ${ }^{35, m m}$ M. S. Neubauer, ${ }^{170}$ M. Neumann, ${ }^{179}$ P. R. Newman, ${ }^{21}$ T. Y. Ng, ${ }^{61 \mathrm{c}}$ Y. S. Ng, ${ }^{19}$ H. D. N. Nguyen, ${ }^{99}$ T. Nguyen Manh, ${ }^{107}$ E. Nibigira, ${ }^{37}$ R. B. Nickerson, ${ }^{131}$ R. Nicolaidou, ${ }^{142}$ J. Nielsen, ${ }^{143}$ N. Nikiforou, ${ }^{11}$ V. Nikolaenko, ${ }^{140,11}$ I. Nikolic-Audit, ${ }^{132}$ K. Nikolopoulos, ${ }^{21}$ P. Nilsson, ${ }^{29}$ Y. Ninomiya ${ }^{79}$ A. Nisati,${ }^{70 a}$ N. Nishu, ${ }^{58 \mathrm{c}}$ R. Nisius, ${ }^{113}$ I. Nitsche, ${ }^{45}$ T. Nitta, ${ }^{176}$ T. Nobe, ${ }^{160}$ Y. Noguchi, ${ }^{83}$ M. Nomachi, ${ }^{129}$ I. Nomidis, ${ }^{132}$ M. A. Nomura, ${ }^{29}$ T. Nooney, ${ }^{90}$ M. Nordberg,${ }^{35}$ N. Norjoharuddeen,${ }^{131}$

T. Novak, ${ }^{89}$ O. Novgorodova, ${ }^{46}$ R. Novotny, ${ }^{138}$ M. Nozaki, ${ }^{79}$ L. Nozka, ${ }^{126}$ K. Ntekas, ${ }^{168}$ E. Nurse, ${ }^{92}$ F. Nuti, ${ }^{102}$

F. G. Oakham, ${ }^{33, e}$ H. Oberlack, ${ }^{113}$ T. Obermann, ${ }^{24}$ J. Ocariz,${ }^{132}$ A. Ochi, ${ }^{80}$ I. Ochoa, ${ }^{38}$ J. P. Ochoa-Ricoux, ${ }^{144 a}$ K. O'Connor, ${ }^{26}$ S. Oda ${ }^{85}$ S. Odaka, ${ }^{79}$ A. Oh,${ }^{98}$ S. H. Oh, ${ }^{47}$ C. C. Ohm, ${ }^{151}$ H. Oide, ${ }^{53 b, 53 a}$ H. Okawa, ${ }^{166}$ Y. Okazaki, ${ }^{83}$ Y. Okumura, ${ }^{160}$ T. Okuyama, ${ }^{79}$ A. Olariu, ${ }^{27 b}$ L. F. Oleiro Seabra, ${ }^{136 a}$ S. A. Olivares Pino, ${ }^{144 a}$ D. Oliveira Damazio, ${ }^{29}$ J. L. Oliver, ${ }^{1}$ M. J. R. Olsson, ${ }^{36}$ A. Olszewski, ${ }^{82}$ J. Olszowska, ${ }^{82}$ D. C. O’Neil, ${ }^{149}$ A. Onofre, ${ }^{136 a, 136 \mathrm{e}}$ K. Onogi, ${ }^{115}$ P. U. E. Onyisi, ${ }^{11}$ H. Oppen, ${ }^{130}$ M. J. Oreglia, ${ }^{36}$ Y. Oren, ${ }^{158}$ D. Orestano,${ }^{72 a, 72 b}$ E. C. Orgill, ${ }^{98}$ N. Orlando, ${ }^{61 b}$ A. A. O'Rourke, ${ }^{44}$ R. S. Orr, ${ }^{164}$ B. Osculati, ${ }^{53 b, 53 a, a}$ V. O'Shea, ${ }^{55}$ R. Ospanov, ${ }^{58 a}$ G. Otero y Garzon, ${ }^{30}$ H. Otono, ${ }^{85}$ M. Ouchrif, ${ }^{34 \mathrm{~d}}$ F. Ould-Saada, ${ }^{130}$ A. Ouraou, ${ }^{142}$ Q. Ouyang, ${ }^{15 a}$ M. Owen, ${ }^{55}$ R. E. Owen, ${ }^{21}$ V. E. Ozcan, ${ }^{12 c}$ N. Ozturk, ${ }^{8}$ J. Pacalt, ${ }^{126}$ H. A. Pacey, ${ }^{31}$ K. Pachal,${ }^{149}$ A. Pacheco Pages,${ }^{14}$ L. Pacheco Rodriguez, ${ }^{142}$ C. Padilla Aranda, ${ }^{14}$ S. Pagan Griso, ${ }^{18}$ M. Paganini, ${ }^{180}$ G. Palacino, ${ }^{63}$ S. Palazzo, ${ }^{40 b, 40 a}$ S. Palestini, ${ }^{35}$ M. Palka, ${ }^{81 b}$ D. Pallin, ${ }^{37}$ I. Panagoulias, ${ }^{10}$ C. E. Pandini, ${ }^{35}$ J. G. Panduro Vazquez, ${ }^{91}$ P. Pani, ${ }^{35}$ G. Panizzo,${ }^{64 a, 64 c}$ L. Paolozzi, ${ }^{52}$ T. D. Papadopoulou, ${ }^{10}$ K. Papageorgiou, ${ }^{9, t}$ A. Paramonov, ${ }^{6}$ D. Paredes Hernandez, ${ }^{61 \mathrm{~b}}$ S. R. Paredes Saenz, ${ }^{131}$ B. Parida, ${ }^{58 \mathrm{c}}$ A. J. Parker, ${ }^{87}$ K. A. Parker, ${ }^{44}$ M. A. Parker, ${ }^{31}$ F. Parodi, ${ }^{53 b, 53 a}$ J. A. Parsons,${ }^{38}$ U. Parzefall,${ }^{50}$ V. R. Pascuzzi, ${ }^{164}$ J. M. P. Pasner, ${ }^{143}$ E. Pasqualucci ${ }^{70 a}$ S. Passaggio,${ }^{53 b}$ F. Pastore, ${ }^{91}$ P. Pasuwan, ${ }^{43 a, 43 b}$ S. Pataraia,${ }^{97}$ J. R. Pater, ${ }^{98}$ A. Pathak, ${ }^{178, f}$ T. Pauly, ${ }^{35}$ B. Pearson, ${ }^{113}$ M. Pedersen, ${ }^{130}$ L. Pedraza Diaz, ${ }^{117}$ S. Pedraza Lopez, ${ }^{171}$ R. Pedro, ${ }^{136 a, 136 b}$ S. V. Peleganchuk, ${ }^{120 b, 120 a}$ O. Penc, ${ }^{137}$ C. Peng, ${ }^{15 d}$ H. Peng, ${ }^{58 a}$ B. S. Peralva, ${ }^{78 a}$ M. M. Perego, ${ }^{142}$ A. P. Pereira Peixoto, ${ }^{136 a}$ D. V. Perepelitsa, ${ }^{29}$ F. Peri, ${ }^{19}$ L. Perini, ${ }^{66 a, 66 b}$ H. Pernegger, ${ }^{35}$ S. Perrella, ${ }^{67,67 b}$ V. D. Peshekhonov, ${ }^{77, a}$ K. Peters, ${ }^{44}$ R. F. Y. Peters, ${ }^{98}$ B. A. Petersen, ${ }^{35}$ T. C. Petersen, ${ }^{39}$ E. Petit, ${ }^{56}$ A. Petridis, ${ }^{1}$ C. Petridou, ${ }^{159}$ P. Petroff, ${ }^{128}$ E. Petrolo, ${ }^{70 a}$ M. Petrov, ${ }^{131}$ F. Petrucci, ${ }^{72 a, 72 b}$ M. Pettee ${ }^{180}$ N. E. Pettersson, ${ }^{100}$ A. Peyaud, ${ }^{142}$ R. Pezoa, ${ }^{144 b}$ T. Pham, ${ }^{102}$ F. H. Phillips, ${ }^{104}$ P. W. Phillips,${ }^{141}$ G. Piacquadio, ${ }^{152}$ E. Pianori, ${ }^{18}$ A. Picazio, ${ }^{100}$ M. A. Pickering, ${ }^{131}$ R. Piegaia ${ }^{30}$ J. E. Pilcher, ${ }^{36}$ A. D. Pilkington, ${ }^{98}$ M. Pinamonti, ${ }^{71 a, 71 b}$ J. L. Pinfold, ${ }^{3}$ M. Pitt, ${ }^{177}$ M-A. Pleier, ${ }^{29}$ V. Pleskot, ${ }^{139}$ E. Plotnikova, ${ }^{77}$ D. Pluth, ${ }^{76}$ P. Podberezko, ${ }^{120 b, 120 a}$ R. Poettgen, ${ }^{94}$ R. Poggi, ${ }^{52}$ L. Poggioli, ${ }^{128}$ I. Pogrebnyak, ${ }^{104}$ D. Pohl, ${ }^{24}$ I. Pokharel, ${ }^{51}$ G. Polesello, ${ }^{68 a}$ A. Poley, ${ }^{44}$ A. Policicchio, ${ }^{40 b 40 \mathrm{a}}$ R. Polifka, ${ }^{35}$ A. Polini, ${ }^{23 \mathrm{~b}}$ C. S. Pollard, ${ }^{44}$ V. Polychronakos, ${ }^{29}$ D. Ponomarenko, ${ }^{110}$ L. Pontecorvo, ${ }^{70 a}$ G. A. Popeneciu, ${ }^{27 d}$ D. M. Portillo Quintero, ${ }^{132}$ S. Pospisil, ${ }^{138}$ K. Potamianos,${ }^{44}$ I. N. Potrap,${ }^{77}$ C. J. Potter, ${ }^{31}$ H. Potti, ${ }^{11}$ T. Poulsen, ${ }^{94}$ J. Poveda, ${ }^{35}$ T. D. Powell, ${ }^{146}$ M. E. Pozo Astigarraga ${ }^{35}$ P. Pralavorio, ${ }^{99}$ S. Prell, ${ }^{76}$ D. Price, ${ }^{98}$ M. Primavera, ${ }^{65 a}$ S. Prince, ${ }^{101}$ N. Proklova, ${ }^{110}$ K. Prokofiev,${ }^{61 \mathrm{c}}$ F. Prokoshin, ${ }^{144 \mathrm{~b}}$ S. Protopopescu, ${ }^{29}$ J. Proudfoot, ${ }^{6}$ M. Przybycien, ${ }^{81 \mathrm{a}}$ A. Puri, ${ }^{170}$ P. Puzo, ${ }^{128}$ J. Qian, ${ }^{103}$ Y. Qin, ${ }^{98}$ A. Quadt,${ }^{51}$ M. Queitsch-Maitland, ${ }^{44}$ A. Qureshi, ${ }^{1}$ P. Rados, ${ }^{102}$ F. Ragusa, ${ }^{66 a, 66 b}$ G. Rahal, ${ }^{95}$ J. A. Raine, ${ }^{98}$ S. Rajagopalan, ${ }^{29}$ A. Ramirez Morales, ${ }^{90}$ T. Rashid, ${ }^{128}$ S. Raspopov, ${ }^{5}$ M. G. Ratti, ${ }^{66 a, 66 b}$ D. M. Rauch, ${ }^{44}$ F. Rauscher, ${ }^{112}$ S. Rave,${ }^{97}$ B. Ravina, ${ }^{146}$ I. Ravinovich, ${ }^{177}$ J. H. Rawling, ${ }^{98}$ M. Raymond, ${ }^{35}$ A. L. Read, ${ }^{130}$ N. P. Readioff, ${ }^{56}$ M. Reale, ${ }^{65 a, 65 b}$ D. M. Rebuzzi ${ }^{68 a, 68 b}$ A. Redelbach, ${ }^{174}$ G. Redlinger, ${ }^{29}$ R. Reece, ${ }^{143}$ R. G. Reed, ${ }^{32 \mathrm{c}}$ K. Reeves, ${ }^{42}$ L. Rehnisch, ${ }^{19}$ J. Reichert, ${ }^{133}$ A. Reiss, ${ }^{97}$ C. Rembser,${ }^{35}$ H. Ren, ${ }^{15 \mathrm{~d}}$ M. Rescigno, ${ }^{70 \mathrm{a}}$ S. Resconi, ${ }^{66 \mathrm{a}}$ E. D. Resseguie, ${ }^{133}$ S. Rettie, ${ }^{172}$ E. Reynolds, ${ }^{21}$ O. L. Rezanova, ${ }^{120 b, 120 a}$ P. Reznicek, ${ }^{139}$ R. Richter, ${ }^{113}$ S. Richter, ${ }^{92}$ E. Richter-Was, ${ }^{81 b}$ O. Ricken, ${ }^{24}$ M. Ridel, ${ }^{132}$ P. Rieck,${ }^{113}$ C. J. Riegel,${ }^{179}$ O. Rifki, ${ }^{44}$ M. Rijssenbeek, ${ }^{152}$ A. Rimoldi, ${ }^{68 a, 68 b}$ M. Rimoldi, ${ }^{20}$ L. Rinaldi, ${ }^{23 b}$ G. Ripellino, ${ }^{151}$ B. Ristić, ${ }^{87}$ E. Ritsch,${ }^{35}$ I. Riu, ${ }^{14}$ J. C. Rivera Vergara, ${ }^{144 a}$ F. Rizatdinova, ${ }^{125}$ E. Rizvi, ${ }^{90}$ C. Rizzi,${ }^{14}$ R. T. Roberts ${ }^{98}$ S. H. Robertson, ${ }^{101, \mathrm{~m}}$ A. Robichaud-Veronneau, ${ }^{101}$ D. Robinson, ${ }^{31}$ 
J. E. M. Robinson, ${ }^{44}$ A. Robson, ${ }^{55}$ E. Rocco,${ }^{97}$ C. Roda,${ }^{69 a, 69 b}$ Y. Rodina, ${ }^{99}$ S. Rodriguez Bosca, ${ }^{171}$ A. Rodriguez Perez, ${ }^{14}$ D. Rodriguez Rodriguez, ${ }^{171}$ A. M. Rodríguez Vera, ${ }^{165 b}$ S. Roe,${ }^{35}$ C. S. Rogan,${ }^{57}$ O. Røhne, ${ }^{130}$ R. Röhrig, ${ }^{113}$ C. P. A. Roland, ${ }^{63}$ J. Roloff, ${ }^{57}$ A. Romaniouk,${ }^{110}$ M. Romano, ${ }^{23 b, 23 a}$ N. Rompotis, ${ }^{88}$ M. Ronzani, ${ }^{121}$ L. Roos, ${ }^{132}$ S. Rosati, ${ }^{70 a}$ K. Rosbach, ${ }^{50}$ P. Rose, ${ }^{143}$ N-A. Rosien, ${ }^{51}$ E. Rossi ${ }^{67 a, 67 b}$ L. P. Rossi, ${ }^{53 b}$ L. Rossini ${ }^{66 a, 66 b}$ J. H. N. Rosten, ${ }^{31}$ R. Rosten, ${ }^{14}$ M. Rotaru, ${ }^{27 b}$ J. Rothberg, ${ }^{145}$ D. Rousseau, ${ }^{128}$ D. Roy ${ }^{32 c}$ A. Rozanov, ${ }^{99}$ Y. Rozen, ${ }^{157}$ X. Ruan,${ }^{32 c}$ F. Rubbo, ${ }^{150}$ F. Rühr, ${ }^{50}$ A. Ruiz-Martinez, ${ }^{33}$ Z. Rurikova, ${ }^{50}$ N. A. Rusakovich, ${ }^{77}$ H. L. Russell, ${ }^{101}$ J. P. Rutherfoord, ${ }^{7}$ N. Ruthmann, ${ }^{35}$ E. M. Rüttinger, ${ }^{44 n}$ Y. F. Ryabov, ${ }^{134}$ M. Rybar, ${ }^{170}$ G. Rybkin, ${ }^{128}$ S. Ryu, ${ }^{6}$ A. Ryzhov, ${ }^{140}$ G. F. Rzehorz,${ }^{51}$ P. Sabatini, ${ }^{51}$ G. Sabato, ${ }^{118}$ S. Sacerdoti, ${ }^{128}$ H. F-W. Sadrozinski, ${ }^{143}$ R. Sadykov, ${ }^{77}$ F. Safai Tehrani, ${ }^{70 a}$ P. Saha, ${ }^{119}$ M. Sahinsoy, ${ }^{59 a}$ A. Sahu, ${ }^{179}$ M. Saimpert, ${ }^{44}$ M. Saito, ${ }^{160}$ T. Saito, ${ }^{160}$ H. Sakamoto, ${ }^{160}$ A. Sakharov, ${ }^{121, g g}$ D. Salamani, ${ }^{52}$ G. Salamanna, ${ }^{72 a, 72 b}$ J. E. Salazar Loyola, ${ }^{144 \mathrm{~b}}$ D. Salek, ${ }^{118}$ P. H. Sales De Bruin, ${ }^{169}$ D. Salihagic, ${ }^{113}$ A. Salnikov, ${ }^{150}$ J. Salt, ${ }^{171}$ D. Salvatore, ${ }^{40 b, 40 a}$ F. Salvatore, ${ }^{153}$ A. Salvucci, ${ }^{61 \mathrm{a}, 61 \mathrm{~b}, 61 \mathrm{c}}$ A. Salzburger, ${ }^{35}$ D. Sammel,${ }^{50}$ D. Sampsonidis, ${ }^{159}$ D. Sampsonidou, ${ }^{159}$ J. Sánchez, ${ }^{171}$

A. Sanchez Pineda,${ }^{64 a, 64 c}$ H. Sandaker ${ }^{130}$ C. O. Sander, ${ }^{44}$ M. Sandhoff,${ }^{179}$ C. Sandoval, ${ }^{22}$ D. P. C. Sankey, ${ }^{141}$ M. Sannino, ${ }^{53 b, 53 a}$ Y. Sano, ${ }^{115}$ A. Sansoni,${ }^{49}$ C. Santoni,${ }^{37}$ H. Santos, ${ }^{136 a}$ I. Santoyo Castillo, ${ }^{153}$ A. Sapronov, ${ }^{77}$ J. G. Saraiva, ${ }^{136 a, 136 \mathrm{~d}}$ O. Sasaki, ${ }^{79}$ K. Sato, ${ }^{166}$ E. Sauvan, ${ }^{5}$ P. Savard,${ }^{164, \mathrm{e}}$ N. Savic, ${ }^{113}$ R. Sawada, ${ }^{160}$ C. Sawyer ${ }^{141}$ L. Sawyer, ${ }^{93, v}$ C. Sbarra, ${ }^{23 b}$ A. Sbrizzi, ${ }^{23 b, 23 a}$ T. Scanlon, ${ }^{92}$ J. Schaarschmidt, ${ }^{145}$ P. Schacht, ${ }^{113}$ B. M. Schachtner, ${ }^{112}$

D. Schaefer, ${ }^{36}$ L. Schaefer, ${ }^{133}$ J. Schaeffer, ${ }^{97}$ S. Schaepe,${ }^{35}$ U. Schäfer,${ }^{97}$ A. C. Schaffer, ${ }^{128}$ D. Schaile, ${ }^{112}$

R. D. Schamberger, ${ }^{152}$ N. Scharmberg, ${ }^{98}$ V. A. Schegelsky, ${ }^{134}$ D. Scheirich, ${ }^{139}$ F. Schenck, ${ }^{19}$ M. Schernau, ${ }^{168}$ C. Schiavi, ${ }^{53 b, 53 a}$ S. Schier, ${ }^{143}$ L. K. Schildgen ${ }^{24}$ Z. M. Schillaci, ${ }^{26}$ E. J. Schioppa,${ }^{35}$ M. Schioppa, ${ }^{40 b, 40 a}$ K. E. Schleicher, ${ }^{50}$ S. Schlenker, ${ }^{35}$ K. R. Schmidt-Sommerfeld, ${ }^{113}$ K. Schmieden, ${ }^{35}$ C. Schmitt, ${ }^{97}$ S. Schmitt, ${ }^{44}$ S. Schmitz, ${ }^{97}$ U. Schnoor, ${ }^{50}$ L. Schoeffel, ${ }^{142}$ A. Schoening, ${ }^{59 b}$ E. Schopf, ${ }^{24}$ M. Schott, ${ }^{97}$ J. F. P. Schouwenberg, ${ }^{117}$ J. Schovancova, ${ }^{35}$ S. Schramm, ${ }^{52}$ A. Schulte, ${ }^{97}$ H-C. Schultz-Coulon, ${ }^{59 a}$ M. Schumacher, ${ }^{50}$ B. A. Schumm, ${ }^{143}$ Ph. Schune, ${ }^{142}$ A. Schwartzman, ${ }^{150}$

T. A. Schwarz, ${ }^{103}$ H. Schweiger, ${ }^{98}$ Ph. Schwemling, ${ }^{142}$ R. Schwienhorst, ${ }^{104}$ A. Sciandra, ${ }^{24}$ G. Sciolla, ${ }^{26}$

M. Scornajenghi, ${ }^{40 b, 40 a}$ F. Scuri, ${ }^{69 a}$ F. Scutti, ${ }^{102}$ L. M. Scyboz, ${ }^{113}$ J. Searcy, ${ }^{103}$ C. D. Sebastiani, ${ }^{70 a, 70 b}$ P. Seema, ${ }^{24}$

S. C. Seidel,${ }^{116}$ A. Seiden,${ }^{143}$ T. Seiss,${ }^{36}$ J. M. Seixas,${ }^{78 b}$ G. Sekhniaidze, ${ }^{67 a}$ K. Sekhon, ${ }^{103}$ S. J. Sekula, ${ }^{41}$ N. Semprini-Cesari, ${ }^{23 b, 23 a}$ S. Sen, ${ }^{47}$ S. Senkin, ${ }^{37}$ C. Serfon, ${ }^{130}$ L. Serin, ${ }^{128}$ L. Serkin, ${ }^{64 a, 64 b}$ M. Sessa, ${ }^{72 a, 72 b}$ H. Severini, ${ }^{124}$ F. Sforza, ${ }^{167}$ A. Sfyrla, ${ }^{52}$ E. Shabalina, ${ }^{51}$ J. D. Shahinian, ${ }^{143}$ N. W. Shaikh,${ }^{43 a, 43 b}$ L. Y. Shan, ${ }^{15 a}$ R. Shang, ${ }^{170}$ J. T. Shank, ${ }^{25}$ M. Shapiro, ${ }^{18}$ A. S. Sharma, ${ }^{1}$ A. Sharma, ${ }^{131}$ P. B. Shatalov, ${ }^{109}$ K. Shaw, ${ }^{153}$ S. M. Shaw, ${ }^{98}$ A. Shcherbakova, ${ }^{134}$ Y. Shen, ${ }^{124}$

N. Sherafati, ${ }^{33}$ A. D. Sherman, ${ }^{25}$ P. Sherwood, ${ }^{92}$ L. Shi, ${ }^{155,00}$ S. Shimizu, ${ }^{80}$ C. O. Shimmin, ${ }^{180}$ M. Shimojima, ${ }^{114}$ I. P. J. Shipsey, ${ }^{131}$ S. Shirabe, ${ }^{85}$ M. Shiyakova, ${ }^{77}$ J. Shlomi, ${ }^{177}$ A. Shmeleva ${ }^{108}$ D. Shoaleh Saadi, ${ }^{107}$ M. J. Shochet, ${ }^{36}$

S. Shojaii, ${ }^{102}$ D. R. Shope, ${ }^{124}$ S. Shrestha, ${ }^{122}$ E. Shulga,${ }^{110}$ P. Sicho,${ }^{137}$ A. M. Sickles,${ }^{170}$ P. E. Sidebo, ${ }^{151}$

E. Sideras Haddad, ${ }^{32 c}$ O. Sidiropoulou, ${ }^{174}$ A. Sidoti, ${ }^{23 b, 23 a}$ F. Siegert, ${ }^{46}$ Dj. Sijacki, ${ }^{16}$ J. Silva, ${ }^{136 a}$ M. Silva Jr., ${ }^{178}$ M. V. Silva Oliveira, ${ }^{78 a}$ S. B. Silverstein, ${ }^{43 a}$ L. Simic,${ }^{77}$ S. Simion, ${ }^{128}$ E. Simioni, ${ }^{97}$ M. Simon, ${ }^{97}$ P. Sinervo, ${ }^{164}$ N. B. Sinev, ${ }^{127}$ M. Sioli, ${ }^{23 b, 23 a}$ G. Siragusa, ${ }^{174}$ I. Siral, ${ }^{103}$ S. Yu. Sivoklokov, ${ }^{111}$ J. Sjölin, ${ }^{43 a, 43 b}$ M. B. Skinner, ${ }^{87}$ P. Skubic, ${ }^{124}$ M. Slater, ${ }^{21}$ T. Slavicek, ${ }^{138}$ M. Slawinska, ${ }^{82}$ K. Sliwa,${ }^{167}$ R. Slovak, ${ }^{139}$ V. Smakhtin, ${ }^{177}$ B. H. Smart, ${ }^{5}$ J. Smiesko, ${ }^{28 a}$ N. Smirnov, ${ }^{110}$ S. Yu. Smirnov, ${ }^{110}$ Y. Smirnov, ${ }^{110}$ L. N. Smirnova, ${ }^{111}$ O. Smirnova, ${ }^{94}$ J. W. Smith,${ }^{51}$ M. N. K. Smith, ${ }^{38}$ R. W. Smith, ${ }^{38}$ M. Smizanska, ${ }^{87}$ K. Smolek, ${ }^{138}$ A. A. Snesarev, ${ }^{108}$ I. M. Snyder, ${ }^{127}$ S. Snyder, ${ }^{29}$ R. Sobie, ${ }^{173, m}$ A. M. Soffa, ${ }^{168}$ A. Soffer, ${ }^{158}$ A. Søgaard, ${ }^{48}$ D. A. Soh, ${ }^{155}$ G. Sokhrannyi, ${ }^{89}$ C. A. Solans Sanchez, ${ }^{35}$ M. Solar, ${ }^{138}$ E. Yu. Soldatov, ${ }^{110}$ U. Soldevila, ${ }^{171}$ A. A. Solodkov ${ }^{140}$ A. Soloshenko, ${ }^{77}$ O. V. Solovyanov, ${ }^{140}$ V. Solovyev, ${ }^{134}$ P. Sommer, ${ }^{146}$ H. Son, ${ }^{167}$ W. Song, ${ }^{141}$

A. Sopczak, ${ }^{138}$ F. Sopkova, ${ }^{28 b}$ D. Sosa, ${ }^{59 b}$ C. L. Sotiropoulou, ${ }^{69 a, 69 b}$ S. Sottocornola, ${ }^{68 a, 68 b}$ R. Soualah,${ }^{64,64 c, p p}$ A. M. Soukharev, ${ }^{120 b, 120 a}$ D. South, ${ }^{44}$ B. C. Sowden, ${ }^{91}$ S. Spagnolo, ${ }^{65 a, 65 b}$ M. Spalla, ${ }^{113}$ M. Spangenberg, ${ }^{175}$ F. Spanò, ${ }^{91}$ D. Sperlich, ${ }^{19}$ F. Spettel, ${ }^{113}$ T. M. Spieker, ${ }^{59 a}$ R. Spighi ${ }^{23 b}$ G. Spigo, ${ }^{35}$ L. A. Spiller, ${ }^{102}$ D. P. Spiteri, ${ }^{55}$ M. Spousta, ${ }^{139}$ A. Stabile, ${ }^{66 a, 66 b}$ R. Stamen ${ }^{59 a}$ S. Stamm, ${ }^{19}$ E. Stanecka,${ }^{82}$ R. W. Stanek, ${ }^{6}$ C. Stanescu, ${ }^{72 a}$ B. Stanislaus, ${ }^{131}$ M. M. Stanitzki ${ }^{44}$ B. Stapf, ${ }^{118}$ S. Stapnes, ${ }^{130}$ E. A. Starchenko, ${ }^{140}$ G. H. Stark ${ }^{36}$ J. Stark, ${ }^{56}$ S. H Stark, ${ }^{39}$ P. Staroba, ${ }^{137}$ P. Starovoitov, ${ }^{59 a}$ S. Stärz, ${ }^{35}$ R. Staszewski, ${ }^{82}$ M. Stegler, ${ }^{44}$ P. Steinberg, ${ }^{29}$ B. Stelzer,${ }^{149}$ H. J. Stelzer, ${ }^{35}$ O. Stelzer-Chilton, ${ }^{165 a}$ H. Stenzel, ${ }^{54}$ T. J. Stevenson, ${ }^{90}$ G. A. Stewart, ${ }^{55}$ M. C. Stockton, ${ }^{127}$ G. Stoicea, ${ }^{27 b}$ P. Stolte, ${ }^{51}$ S. Stonjek, ${ }^{113}$ A. Straessner, ${ }^{46}$ J. Strandberg, ${ }^{151}$ S. Strandberg, ${ }^{43 a, 43 b}$ M. Strauss, ${ }^{124}$ P. Strizenec, ${ }^{28 b}$ R. Ströhmer, ${ }^{174}$ D. M. Strom, ${ }^{127}$ R. Stroynowski, ${ }^{41}$ A. Strubig, ${ }^{48}$ S. A. Stucci, ${ }^{29}$ B. Stugu, ${ }^{17}$ J. Stupak, ${ }^{124}$ N. A. Styles,${ }^{44}$ D. Su, ${ }^{150}$ J. Su, ${ }^{135}$ S. Suchek, ${ }^{59 a}$ Y. Sugaya,${ }^{129}$ M. Suk, ${ }^{138}$ V. V. Sulin, ${ }^{108}$ D. M. S. Sultan, ${ }^{52}$ S. Sultansoy, ${ }^{4 c}$ T. Sumida, ${ }^{83}$ S. Sun, ${ }^{103}$ X. Sun, ${ }^{3}$ K. Suruliz, ${ }^{153}$ C. J. E. Suster, ${ }^{154}$ M. R. Sutton, ${ }^{153}$ S. Suzuki, ${ }^{79}$ M. Svatos, ${ }^{137}$ M. Swiatlowski, ${ }^{36}$ S. P. Swift, ${ }^{2}$ A. Sydorenko, ${ }^{97}$ I. Sykora, ${ }^{28 a}$ T. Sykora, ${ }^{139}$ 
D. Ta ${ }^{97}$ K. Tackmann, ${ }^{44, q q}$ J. Taenzer, ${ }^{158}$ A. Taffard, ${ }^{168}$ R. Tafirout, ${ }^{165 a}$ E. Tahirovic,${ }^{90}$ N. Taiblum, ${ }^{158}$ H. Takai, ${ }^{29}$ R. Takashima, ${ }^{84}$ E. H. Takasugi, ${ }^{113}$ K. Takeda,${ }^{80}$ T. Takeshita, ${ }^{147}$ Y. Takubo, ${ }^{79}$ M. Talby, ${ }^{99}$ A. A. Talyshev,${ }^{120 b, 120 a}$ J. Tanaka, ${ }^{160}$ M. Tanaka, ${ }^{162}$ R. Tanaka, ${ }^{128}$ R. Tanioka, ${ }^{80}$ B. B. Tannenwald, ${ }^{122}$ S. Tapia Araya, ${ }^{144 \mathrm{~b}}$ S. Tapprogge, ${ }^{97}$ A. Tarek Abouelfadl Mohamed, ${ }^{132}$ S. Tarem, ${ }^{157}$ G. Tarna, ${ }^{27 b, q}$ G. F. Tartarelli, ${ }^{66 a}$ P. Tas, ${ }^{139}$ M. Tasevsky,${ }^{137}$ T. Tashiro, ${ }^{83}$ E. Tassi, ${ }^{40 b, 40 a}$ A. Tavares Delgado, ${ }^{136 a, 136 b}$ Y. Tayalati, ${ }^{34 \mathrm{e}}$ A. C. Taylor, ${ }^{116}$ A. J. Taylor, ${ }^{48}$ G. N. Taylor, ${ }^{102}$ P. T. E. Taylor, ${ }^{102}$ W. Taylor, ${ }^{165 b}$ A. S. Tee, ${ }^{87}$ P. Teixeira-Dias, ${ }^{91}$ H. Ten Kate, ${ }^{35}$ P. K. Teng, ${ }^{155}$ J. J. Teoh, ${ }^{129}$ F. Tepel, ${ }^{179}$ S. Terada, ${ }^{79}$ K. Terashi, ${ }^{160}$ J. Terron, ${ }^{96}$ S. Terzo, ${ }^{14}$ M. Testa, ${ }^{49}$ R. J. Teuscher, ${ }^{164, \mathrm{~m}}$ S. J. Thais, ${ }^{180}$ T. Theveneaux-Pelzer, ${ }^{44}$ F. Thiele, ${ }^{39}$ J. P. Thomas, ${ }^{21}$ A. S. Thompson, ${ }^{55}$ P. D. Thompson, ${ }^{21}$ L. A. Thomsen, ${ }^{180}$ E. Thomson, ${ }^{133}$ Y. Tian,${ }^{38}$ R. E. Ticse Torres,${ }^{51}$ V. O. Tikhomirov, ${ }^{108, r r}$ Yu. A. Tikhonov, ${ }^{120 b, 120 \mathrm{a}}$ S. Timoshenko, ${ }^{110}$ P. Tipton, ${ }^{180}$ S. Tisserant, ${ }^{99} \mathrm{~K}$. Todome, ${ }^{162}$ S. Todorova-Nova, ${ }^{5}$ S. Todt,${ }^{46}$ J. Tojo, ${ }^{85}$ S. Tokár, ${ }^{28 a}$ K. Tokushuku, ${ }^{79}$ E. Tolley, ${ }^{122}$ K. G. Tomiwa, ${ }^{32 c}$ M. Tomoto, ${ }^{115}$ L. Tompkins, ${ }^{150, f f}$ K. Toms, ${ }^{116}$ B. Tong,${ }^{57}$ P. Tornambe,${ }^{50}$ E. Torrence, ${ }^{127}$ H. Torres, ${ }^{46}$ E. Torró Pastor, ${ }^{145}$ C. Tosciri, ${ }^{131}$ J. Toth, ${ }^{99, s s}$ F. Touchard, ${ }^{99}$ D. R. Tovey, ${ }^{146}$ C. J. Treado, ${ }^{121}$ T. Trefzger, ${ }^{174}$ F. Tresoldi, ${ }^{153}$ A. Tricoli, ${ }^{29}$ I. M. Trigger, ${ }^{165 a}$ S. Trincaz-Duvoid, ${ }^{132}$ M. F. Tripiana, ${ }^{14}$ W. Trischuk, ${ }^{164}$ B. Trocmé,${ }^{56}$ A. Trofymov, ${ }^{128}$ C. Troncon, ${ }^{66 a}$ M. Trovatelli, ${ }^{173}$ F. Trovato, ${ }^{153}$ L. Truong, ${ }^{32 b}$ M. Trzebinski, ${ }^{82}$ A. Trzupek, ${ }^{82}$ F. Tsai, ${ }^{44}$ J. C-L. Tseng, ${ }^{131}$ P. V. Tsiareshka,${ }^{105}$ N. Tsirintanis, ${ }^{9}$ V. Tsiskaridze, ${ }^{152}$ E. G. Tskhadadze, ${ }^{156 \mathrm{a}}$ I. I. Tsukerman, ${ }^{109}$ V. Tsulaia, ${ }^{18}$ S. Tsuno, ${ }^{79}$ D. Tsybychev $,{ }^{152} \mathrm{Y} . \mathrm{Tu},{ }^{61 \mathrm{~b}}$ A. Tudorache, ${ }^{27 b}$ V. Tudorache, ${ }^{27 b}$ T. T. Tulbure, ${ }^{27 a}$ A. N. Tuna, ${ }^{57}$ S. Turchikhin, ${ }^{77}$ D. Turgeman, ${ }^{177}$ I. Turk Cakir, ${ }^{4 b, t t}$ R. Turra, ${ }^{66 \mathrm{a}}$ P. M. Tuts, ${ }^{38}$ E. Tzovara, ${ }^{97}$ G. Ucchielli, ${ }^{23 b, 23 a}$ I. Ueda,${ }^{79}$ M. Ughetto, ${ }^{43 a, 43 b}$ F. Ukegawa, ${ }^{166}$ G. Unal, ${ }^{35}$ A. Undrus, ${ }^{29}$ G. Unel, ${ }^{168}$ F. C. Ungaro, ${ }^{102}$ Y. Unno, ${ }^{79}$ K. Uno, ${ }^{160}$ J. Urban,${ }^{28 b}$ P. Urquijo, ${ }^{102}$ P. Urrejola, ${ }^{97}$ G. Usai, ${ }^{8}$ J. Usui,${ }^{79}$ L. Vacavant, ${ }^{99}$ V. Vacek, ${ }^{138}$ B. Vachon, ${ }^{101}$ K. O. H. Vadla, ${ }^{130}$ A. Vaidya,${ }^{92}$ C. Valderanis, ${ }^{112}$ E. Valdes Santurio, ${ }^{43 a, 43 b}$ M. Valente, ${ }^{52}$ S. Valentinetti, ${ }^{23 b, 23 a}$ A. Valero, ${ }^{171}$ L. Valéry, ${ }^{44}$ R. A. Vallance, ${ }^{21}$ A. Vallier, ${ }^{5}$ J. A. Valls Ferrer, ${ }^{171}$ T. R. Van Daalen, ${ }^{14}$ W. Van Den Wollenberg, ${ }^{118}$ H. Van der Graaf, ${ }^{118}$ P. Van Gemmeren, ${ }^{6}$ J. Van Nieuwkoop, ${ }^{149}$ I. Van Vulpen, ${ }^{118}$ M. C. van Woerden, ${ }^{118}$ M. Vanadia, ${ }^{71 a, 71 b}$ W. Vandelli, ${ }^{35}$ A. Vaniachine, ${ }^{163}$ P. Vankov, ${ }^{118}$ R. Vari, ${ }^{70 a}$ E. W. Varnes, ${ }^{7}$ C. Varni, ${ }^{53 b, 53 a}$ T. Varol, ${ }^{41}$ D. Varouchas, ${ }^{128}$ A. Vartapetian, ${ }^{8}$ K. E. Varvell, ${ }^{154}$ G. A. Vasquez, ${ }^{144 b}$ J. G. Vasquez ${ }^{180}$ F. Vazeille, ${ }^{37}$ D. Vazquez Furelos, ${ }^{14}$ T. Vazquez Schroeder, ${ }^{101}$ J. Veatch, ${ }^{51}$ V. Vecchio, ${ }^{72 a, 72 b}$ L. M. Veloce, ${ }^{164}$ F. Veloso, ${ }^{136 a, 136 c}$ S. Veneziano, ${ }^{70 a}$ A. Ventura, ${ }^{65 a, 65 b}$ M. Venturi, ${ }^{173}$ N. Venturi, ${ }^{35}$ V. Vercesi, ${ }^{68 a}$ M. Verducci, ${ }^{72 a, 72 b}$ C. M. Vergel Infante, ${ }^{76}$ W. Verkerke, ${ }^{118}$ A. T. Vermeulen, ${ }^{118}$ J. C. Vermeulen, ${ }^{118}$ M. C. Vetterli, ${ }^{149, e}$ N. Viaux Maira, ${ }^{144 b}$ O. Viazlo, ${ }^{94}$ I. Vichou, ${ }^{170, a}$ T. Vickey, ${ }^{146}$ O. E. Vickey Boeriu, ${ }^{146}$ G. H. A. Viehhauser, ${ }^{131}$ S. Viel, ${ }^{18}$ L. Vigani, ${ }^{131}$ M. Villa, ${ }^{23 b, 23 a}$ M. Villaplana Perez, ${ }^{66 a, 66 b}$ E. Vilucchi, ${ }^{49}$ M. G. Vincter, ${ }^{33}$ V. B. Vinogradov, ${ }^{77}$ A. Vishwakarma ${ }^{44}$ C. Vittori, ${ }^{23 b, 23 a}$ I. Vivarelli, ${ }^{153}$ S. Vlachos,${ }^{10}$ M. Vogel, ${ }^{179}$ P. Vokac, ${ }^{138}$ G. Volpi,${ }^{14}$ S. E. von Buddenbrock, ${ }^{32 \mathrm{c}}$ E. Von Toerne, ${ }^{24}$ V. Vorobel, ${ }^{139}$ K. Vorobev, ${ }^{110}$ M. Vos, ${ }^{171}$ J. H. Vossebeld, ${ }^{88}$ N. Vranjes, ${ }^{16}$ M. Vranjes Milosavljevic, ${ }^{16}$ V. Vrba, ${ }^{138}$ M. Vreeswijk, ${ }^{118}$ T. Šfiligoj, ${ }^{89}$ R. Vuillermet, ${ }^{35}$ I. Vukotic, ${ }^{36}$ T. Ženišs, ${ }^{28 a}$ L. Živković, ${ }^{16}$ P. Wagner, ${ }^{24}$ W. Wagner,${ }^{179}$ J. Wagner-Kuhr, ${ }^{112} \mathrm{H}$. Wahlberg, ${ }^{86} \mathrm{~S}$. Wahrmund, ${ }^{46} \mathrm{~K}$. Wakamiya ${ }^{80}$ V. M. Walbrecht, ${ }^{113}$ J. Walder,${ }^{87}$ R. Walker, ${ }^{112}$ W. Walkowiak, ${ }^{148}$ V. Wallangen, ${ }^{43 a, 43 b}$ A. M. Wang, ${ }^{57}$ C. Wang, ${ }^{58 b, q}$ F. Wang, ${ }^{178}$ H. Wang, ${ }^{18}$ H. Wang, ${ }^{3}$ J. Wang, ${ }^{154}$ J. Wang, ${ }^{59 b}$ P. Wang, ${ }^{41}$ Q. Wang, ${ }^{124}$ R.-J. Wang, ${ }^{132}$ R. Wang, ${ }^{58 a}$ R. Wang, ${ }^{6}$ S. M. Wang, ${ }^{155}$ W. T. Wang, ${ }^{58 \mathrm{a}}$ W. Wang, ${ }^{155, \text { uu }}$ W. X. Wang, ${ }^{58, \text {,vv }}$ Y. Wang, ${ }^{58 \mathrm{a}}$ Z. Wang, ${ }^{58 \mathrm{c}}$ C. Wanotayaroj, ${ }^{44}$

A. Warburton, ${ }^{101}$ C. P. Ward, ${ }^{31}$ D. R. Wardrope, ${ }^{92}$ A. Washbrook, ${ }^{48}$ P. M. Watkins, ${ }^{21}$ A. T. Watson, ${ }^{21}$ M. F. Watson, ${ }^{21}$ G. Watts, ${ }^{145}$ S. Watts, ${ }^{98}$ B. M. Waugh, ${ }^{92}$ A. F. Webb, ${ }^{11}$ S. Webb,${ }^{97}$ C. Weber, ${ }^{180}$ M. S. Weber, ${ }^{20}$ S. A. Weber, ${ }^{33}$ S. M. Weber, ${ }^{59 a}$ J. S. Webster, ${ }^{6}$ A. R. Weidberg, ${ }^{131}$ B. Weinert, ${ }^{63}$ J. Weingarten, ${ }^{51}$ M. Weirich, ${ }^{97}$ C. Weiser, ${ }^{50}$ P. S. Wells,${ }^{35}$ T. Wenaus, ${ }^{29}$ T. Wengler, ${ }^{35}$ S. Wenig, ${ }^{35}$ N. Wermes, ${ }^{24}$ M. D. Werner,${ }^{76}$ P. Werner, ${ }^{35}$ M. Wessels, ${ }^{59 a}$ T. D. Weston, ${ }^{20}$ K. Whalen, ${ }^{127}$ N. L. Whallon, ${ }^{145}$ A. M. Wharton, ${ }^{87}$ A. S. White, ${ }^{103}$ A. White, ${ }^{8}$ M. J. White, ${ }^{1}$ R. White, ${ }^{144 b}$ D. Whiteson, ${ }^{168}$ B. W. Whitmore, ${ }^{87}$ F. J. Wickens, ${ }^{141}$ W. Wiedenmann, ${ }^{178}$ M. Wielers, ${ }^{141}$ C. Wiglesworth, ${ }^{39}$ L. A. M. Wiik-Fuchs, ${ }^{50}$ A. Wildauer, ${ }^{113}$ F. Wilk, ${ }^{98}$ H. G. Wilkens, ${ }^{35}$ L. J. Wilkins, ${ }^{91}$ H. H. Williams, ${ }^{133}$ S. Williams, ${ }^{31}$ C. Willis,${ }^{104}$ S. Willocq, ${ }^{100}$ J. A. Wilson, ${ }^{21}$ I. Wingerter-Seez, ${ }^{5}$ E. Winkels,${ }^{153}$ F. Winklmeier, ${ }^{127}$ O. J. Winston, ${ }^{153}$ B. T. Winter, ${ }^{24}$ M. Wittgen, ${ }^{150}$ M. Wobisch, ${ }^{93}$ A. Wolf,${ }^{97}$ T. M. H. Wolf, ${ }^{118}$ R. Wolff, ${ }^{99}$ M. W. Wolter, ${ }^{82}$ H. Wolters, ${ }^{136 a, 136 c}$ V. W. S. Wong, ${ }^{172}$ N. L. Woods, ${ }^{143}$ S. D. Worm, ${ }^{21}$ B. K. Wosiek, ${ }^{82}$ K. W. Woźniak, ${ }^{82}$ K. Wraight, ${ }^{55}$ M. Wu ${ }^{36}$ S. L. Wu, ${ }^{178}$ X. Wu, ${ }^{52}$ Y. Wu, ${ }^{58 a}$ T. R. Wyatt, ${ }^{98}$ B. M. Wynne, ${ }^{48}$ S. Xella,${ }^{39}$ Z. Xi,${ }^{103}$ L. Xia, ${ }^{175}$ D. Xu, ${ }^{15 a}$ H. Xu, ${ }^{58 a}$ L. Xu, ${ }^{29}$ T. Xu, ${ }^{142}$ W. Xu, ${ }^{103}$ B. Yabsley, ${ }^{154}$ S. Yacoob, ${ }^{32 a}$ K. Yajima, ${ }^{129}$ D. P. Yallup, ${ }^{92}$ D. Yamaguchi, ${ }^{162}$ Y. Yamaguchi, ${ }^{162}$ A. Yamamoto, ${ }^{79}$ T. Yamanaka, ${ }^{160}$ F. Yamane ${ }^{80}$ M. Yamatani, ${ }^{160}$ T. Yamazaki ${ }^{160}$ Y. Yamazaki,${ }^{80}$ Z. Yan, ${ }^{25}$ H. J. Yang,${ }^{58 c, 58 d}$ H. T. Yang, ${ }^{18}$ S. Yang ${ }^{75}$ Y. Yang, ${ }^{160}$ Z. Yang, ${ }^{17}$ W-M. Yao, ${ }^{18}$ Y. C. Yap, ${ }^{44}$ Y. Yasu, ${ }^{79}$ E. Yatsenko, ${ }^{58 c, 58 d}$ J. Ye, ${ }^{41}$ S. Ye, ${ }^{29}$ I. Yeletskikh, ${ }^{77}$ E. Yigitbasi, ${ }^{25}$ 
E. Yildirim, ${ }^{97}$ K. Yorita, ${ }^{176}$ K. Yoshihara, ${ }^{133}$ C. J. S. Young, ${ }^{35}$ C. Young, ${ }^{150}$ J. Yu, ${ }^{8}$ J. Yu, ${ }^{76}$ X. Yue, ${ }^{59 a}$ S. P. Y. Yuen, ${ }^{24}$ I. Yusuff, ${ }^{31, w w}$ B. Zabinski, ${ }^{82}$ G. Zacharis, ${ }^{10}$ E. Zaffaroni, ${ }^{52}$ R. Zaidan, ${ }^{14}$ A. M. Zaitsev, ${ }^{140,11}$ N. Zakharchuk, ${ }^{44}$ J. Zalieckas, ${ }^{17}$ S. Zambito, ${ }^{57}$ D. Zanzi, ${ }^{35}$ D. R. Zaripovas, ${ }^{55}$ S. V. Zeißner, ${ }^{45}$ C. Zeitnitz, ${ }^{179}$ G. Zemaityte, ${ }^{131}$ J. C. Zeng, ${ }^{170}$ Q. Zeng, ${ }^{150}$ O. Zenin, ${ }^{140}$ D. Zerwas, ${ }^{128}$ M. Zgubič, ${ }^{131}$ D. F. Zhang, ${ }^{58 b}$ D. Zhang, ${ }^{103}$ F. Zhang, ${ }^{178}$ G. Zhang, ${ }^{58 a, v v}$ H. Zhang, ${ }^{15 c}$ J. Zhang, ${ }^{6}$ L. Zhang, ${ }^{50}$ L. Zhang, ${ }^{58 \mathrm{a}}$ M. Zhang, ${ }^{170}$ P. Zhang, ${ }^{15 \mathrm{c}}$ R. Zhang, ${ }^{58 \mathrm{a}, \mathrm{q}}$ R. Zhang, ${ }^{24} \mathrm{X}$. Zhang, ${ }^{58 \mathrm{~b}} \mathrm{Y}$. Zhang, ${ }^{15 \mathrm{~d}} \mathrm{Z}$. Zhang, ${ }^{128}$ P. Zhao, ${ }^{47}$ X. Zhao, ${ }^{41}$ Y. Zhao, ${ }^{58 b, 128, y}$ Z. Zhao, ${ }^{58 a}$ A. Zhemchugov, ${ }^{77}$ B. Zhou, ${ }^{103}$ C. Zhou, ${ }^{178}$ L. Zhou, ${ }^{41}$ M. S. Zhou, ${ }^{15 d}$ M. Zhou, ${ }^{152}$ N. Zhou, ${ }^{58 c}$ Y. Zhou, ${ }^{7}$ C. G. Zhu, ${ }^{58 b}$ H. L. Zhu, ${ }^{58 a}$ H. Zhu, ${ }^{15 a}$ J. Zhu, ${ }^{103}$ Y. Zhu, ${ }^{58 a}$ X. Zhuang, ${ }^{15 a}$ K. Zhukov, ${ }^{108}$ V. Zhulanov, ${ }^{120 b, 120 a}$ A. Zibell, ${ }^{174}$ D. Zieminska, ${ }^{63}$ N. I. Zimine, ${ }^{77}$ S. Zimmermann, ${ }^{50}$ Z. Zinonos, ${ }^{113}$ M. Zinser, ${ }^{97}$ M. Ziolkowski, ${ }^{148}$ G. Zobernig, ${ }^{178}$ A. Zoccoli, ${ }^{23 b, 23 a}$ K. Zoch, ${ }^{51}$ T. G. Zorbas, ${ }^{146}$ R. Zou, ${ }^{36}$ M. Zur Nedden, ${ }^{19}$ and L. Zwalinski ${ }^{35}$

(ATLAS Collaboration)

${ }^{1}$ Department of Physics, University of Adelaide, Adelaide, Australia

${ }^{2}$ Physics Department, SUNY Albany, Albany, New York, USA

${ }^{3}$ Department of Physics, University of Alberta, Edmonton, Alberta, Canada

${ }^{4 a}$ Department of Physics, Ankara University, Ankara, Turkey

${ }^{4 \mathrm{~b}}$ Istanbul Aydin University, Istanbul, Turkey

${ }^{4 \mathrm{c}}$ Division of Physics, TOBB University of Economics and Technology, Ankara, Turkey

${ }^{5}$ LAPP, Université Grenoble Alpes, Université Savoie Mont Blanc, CNRS/IN2P3, Annecy, France

${ }^{6}$ High Energy Physics Division, Argonne National Laboratory, Argonne, Illinois, USA

${ }^{7}$ Department of Physics, University of Arizona, Tucson, Azizona, USA

${ }^{8}$ Department of Physics, University of Texas at Arlington, Arlington, Texas, USA

${ }^{9}$ Physics Department, National and Kapodistrian University of Athens, Athens, Greece

${ }^{10}$ Physics Department, National Technical University of Athens, Zografou, Greece

${ }^{11}$ Department of Physics, University of Texas at Austin, Austin, Texas, USA

${ }^{12 a}$ Bahcesehir University, Faculty of Engineering and Natural Sciences, Istanbul, Turkey

${ }^{12 \mathrm{~b}}$ Istanbul Bilgi University, Faculty of Engineering and Natural Sciences, Istanbul, Turkey

${ }^{12 \mathrm{c}}$ Department of Physics, Bogazici University, Istanbul, Turkey

${ }^{12 \mathrm{~d} D e p a r t m e n t}$ of Physics Engineering, Gaziantep University, Gaziantep, Turkey

${ }^{13}$ Institute of Physics, Azerbaijan Academy of Sciences, Baku, Azerbaijan

${ }^{14}$ Institut de Física d'Altes Energies (IFAE), Barcelona Institute of Science and Technology, Barcelona, Spain

${ }^{15 a}$ Institute of High Energy Physics, Chinese Academy of Sciences, Beijing, China

${ }^{15 \mathrm{~b}}$ Physics Department, Tsinghua University, Beijing, China

${ }^{15 \mathrm{c}}$ Department of Physics, Nanjing University, Nanjing, China

${ }^{15 \mathrm{~d}}$ University of Chinese Academy of Science (UCAS), Beijing, China

${ }^{16}$ Institute of Physics, University of Belgrade, Belgrade, Serbia

${ }^{17}$ Department for Physics and Technology, University of Bergen, Bergen, Norway

${ }^{18}$ Physics Division, Lawrence Berkeley National Laboratory and University of California, Berkeley, California, USA

${ }^{19}$ Institut für Physik, Humboldt Universität zu Berlin, Berlin, Germany

${ }^{20}$ Albert Einstein Center for Fundamental Physics and Laboratory for High Energy Physics, University of Bern, Bern, Switzerland

${ }^{21}$ School of Physics and Astronomy, University of Birmingham, Birmingham, United Kingdom

${ }^{22}$ Centro de Investigaciónes, Universidad Antonio Nariño, Bogota, Colombia

${ }^{23 a}$ Dipartimento di Fisica e Astronomia, Università di Bologna, Bologna, Italy ${ }^{23 \mathrm{~b}}$ INFN Sezione di Bologna, Italy

${ }^{24}$ Physikalisches Institut, Universität Bonn, Bonn, Germany

${ }^{25}$ Department of Physics, Boston University, Boston, Massachusetts, USA

${ }^{26}$ Department of Physics, Brandeis University, Waltham, Massachusetts, USA

${ }^{27}$ Transilvania University of Brasov, Brasov, Romania

${ }^{27 \mathrm{~b}}$ Horia Hulubei National Institute of Physics and Nuclear Engineering, Bucharest, Romania

${ }^{27 \mathrm{c}}$ Department of Physics, Alexandru Ioan Cuza University of Iasi, Iasi, Romania

${ }^{27 \mathrm{~d}}$ National Institute for Research and Development of Isotopic and Molecular Technologies, Physics Department, Cluj-Napoca, Romania

${ }^{27 \mathrm{e}}$ University Politehnica Bucharest, Bucharest, Romania 


\footnotetext{
${ }^{27 \mathrm{f}}$ West University in Timisoara, Timisoara, Romania

${ }^{28 a}$ Faculty of Mathematics, Physics and Informatics, Comenius University, Bratislava, Slovak Republic

${ }^{28 \mathrm{~b}}$ Department of Subnuclear Physics, Institute of Experimental Physics of the Slovak Academy of Sciences,

Kosice, Slovak Republic

${ }^{29}$ Physics Department, Brookhaven National Laboratory, Upton, New York, USA

${ }^{30}$ Departamento de Física, Universidad de Buenos Aires, Buenos Aires, Argentina

${ }^{31}$ Cavendish Laboratory, University of Cambridge, Cambridge, United Kingdom

${ }^{32 \mathrm{a}}$ Department of Physics, University of Cape Town, Cape Town, South Africa

${ }^{32 \mathrm{~b}}$ Department of Mechanical Engineering Science, University of Johannesburg, Johannesburg, South Africa

${ }^{32 \mathrm{c}}$ School of Physics, University of the Witwatersrand, Johannesburg, South Africa

${ }^{33}$ Department of Physics, Carleton University, Ottawa, Ontario, Canada

${ }^{34 a}$ Faculté des Sciences Ain Chock, Réseau Universitaire de Physique des Hautes Energies-Université Hassan II, Casablanca, Morocco

${ }^{34 \mathrm{~b}}$ Centre National de l'Energie des Sciences Techniques Nucleaires (CNESTEN), Rabat, Morocco

${ }^{34 \mathrm{c}}$ Faculté des Sciences Semlalia, Université Cadi Ayyad, LPHEA-Marrakech, Morocco

${ }^{34 \mathrm{~d}}$ Faculté des Sciences, Université Mohamed Premier and LPTPM, Oujda, Morocco

${ }^{34 \mathrm{e}}$ Faculté des sciences, Université Mohammed V, Rabat, Morocco

${ }^{35}$ CERN, Geneva, Switzerland

${ }^{36}$ Enrico Fermi Institute, University of Chicago, Chicago, Illinois, USA

${ }^{37}$ LPC, Université Clermont Auvergne, CNRS/IN2P3, Clermont-Ferrand, France

${ }^{38}$ Nevis Laboratory, Columbia University, Irvington, New York, USA

${ }^{39}$ Niels Bohr Institute, University of Copenhagen, Copenhagen, Denmark

${ }^{40 \mathrm{a}}$ Dipartimento di Fisica, Università della Calabria, Rende, Italy

${ }^{40 \mathrm{~b}}$ INFN Gruppo Collegato di Cosenza, Laboratori Nazionali di Frascati, Italy

${ }^{41}$ Physics Department, Southern Methodist University, Dallas, Texas, USA

${ }^{42}$ Physics Department, University of Texas at Dallas, Richardson, Texas, USA

${ }^{43 a}$ Department of Physics, Stockholm University, Sweden

${ }^{43 \mathrm{~b}}$ Oskar Klein Centre, Stockholm, Sweden

${ }^{44}$ Deutsches Elektronen-Synchrotron DESY, Hamburg and Zeuthen, Germany

${ }^{45}$ Lehrstuhl für Experimentelle Physik IV, Technische Universität Dortmund, Dortmund, Germany

${ }^{46}$ Institut für Kern- und Teilchenphysik, Technische Universität Dresden, Dresden, Germany

${ }^{47}$ Department of Physics, Duke University, Durham, North Carolina, USA

${ }^{48}$ SUPA-School of Physics and Astronomy, University of Edinburgh, Edinburgh, United Kingdom

${ }^{49}$ INFN e Laboratori Nazionali di Frascati, Frascati, Italy

${ }^{50}$ Physikalisches Institut, Albert-Ludwigs-Universität Freiburg, Freiburg, Germany

${ }^{51}$ II. Physikalisches Institut, Georg-August-Universität Göttingen, Göttingen, Germany

${ }^{52}$ Département de Physique Nucléaire et Corpusculaire, Université de Genève, Genève, Switzerland

${ }^{53}$ Dipartimento di Fisica, Università di Genova, Genova, Italy

${ }^{53 \mathrm{~b}}$ INFN Sezione di Genova, Italy

${ }^{54}$ II. Physikalisches Institut, Justus-Liebig-Universität Giessen, Giessen, Germany

${ }^{55}$ SUPA-School of Physics and Astronomy, University of Glasgow, Glasgow, United Kingdom

${ }^{56}$ LPSC, Université Grenoble Alpes, CNRS/IN2P3, Grenoble INP, Grenoble, France

${ }^{57}$ Laboratory for Particle Physics and Cosmology, Harvard University, Cambridge, Massachusetts, USA

${ }^{58 \mathrm{a}}$ Department of Modern Physics and State Key Laboratory of Particle Detection and Electronics,

University of Science and Technology of China, Hefei, China

${ }^{58 \mathrm{~b}}$ Institute of Frontier and Interdisciplinary Science and Key Laboratory of Particle Physics and Particle Irradiation (MOE), Shandong University, Qingdao, China

${ }^{58 \mathrm{c}}$ School of Physics and Astronomy, Shanghai Jiao Tong University, KLPPAC-MoE, SKLPPC, Shanghai, China

${ }^{58 \mathrm{~d}}$ Tsung-Dao Lee Institute, Shanghai, China

${ }^{59 a}$ Kirchhoff-Institut für Physik, Ruprecht-Karls-Universität Heidelberg, Heidelberg, Germany

${ }^{59 \mathrm{~b}}$ Physikalisches Institut, Ruprecht-Karls-Universität Heidelberg, Heidelberg, Germany

${ }^{60}$ Faculty of Applied Information Science, Hiroshima Institute of Technology, Hiroshima, Japan

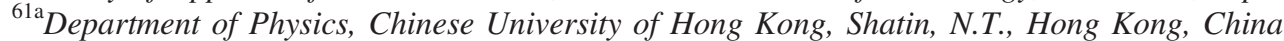

${ }^{61 b}$ Department of Physics, University of Hong Kong, Hong Kong, China

${ }^{61 \mathrm{c} D e p a r t m e n t}$ of Physics and Institute for Advanced Study, Hong Kong University of Science and Technology, Clear Water Bay, Kowloon, Hong Kong, China

${ }^{62}$ Department of Physics, National Tsing Hua University, Hsinchu, Taiwan

${ }^{63}$ Department of Physics, Indiana University, Bloomington, Indiana, USA
} 
${ }^{64 \mathrm{a}}$ INFN Gruppo Collegato di Udine, Sezione di Trieste, Udine, Italy

${ }^{64 \mathrm{~b}}$ ICTP, Trieste, Italy

${ }^{64 \mathrm{c}}$ Dipartimento di Chimica, Fisica e Ambiente, Università di Udine, Udine, Italy

${ }^{65 \mathrm{a}}$ INFN Sezione di Lecce, Italy

${ }^{65 \mathrm{~b}}$ Dipartimento di Matematica e Fisica, Università del Salento, Lecce, Italy

${ }^{66 a}$ INFN Sezione di Milano, Italy

${ }^{66 \mathrm{~b}}$ Dipartimento di Fisica, Università di Milano, Milano, Italy

${ }^{67 a}$ INFN Sezione di Napoli, Italy

${ }^{67 \mathrm{~b}}$ Dipartimento di Fisica, Università di Napoli, Napoli, Italy

${ }^{68 \mathrm{a}}$ INFN Sezione di Pavia, Italy

${ }^{68 \mathrm{~b}}$ Dipartimento di Fisica, Università di Pavia, Pavia, Italy

${ }^{69 a}$ INFN Sezione di Pisa, Italy

${ }^{69 \mathrm{~b}}$ Dipartimento di Fisica E. Fermi, Università di Pisa, Pisa, Italy

${ }^{70 a}$ INFN Sezione di Roma, Italy

${ }^{70 \mathrm{~b}}$ Dipartimento di Fisica, Sapienza Università di Roma, Roma, Italy

${ }^{71 \mathrm{a}}$ INFN Sezione di Roma Tor Vergata, Italy

${ }^{71 \mathrm{~b}}$ Dipartimento di Fisica, Università di Roma Tor Vergata, Roma, Italy

${ }^{72 \mathrm{a}}$ INFN Sezione di Roma Tre, Italy

${ }^{72 b}$ Dipartimento di Matematica e Fisica, Università Roma Tre, Roma, Italy

${ }^{73 a}$ INFN-TIFPA, Italy

${ }^{73 b}$ Università degli Studi di Trento, Trento, Italy

${ }^{74}$ Institut für Astro- und Teilchenphysik, Leopold-Franzens-Universität, Innsbruck, Austria

${ }^{75}$ University of Iowa, Iowa City, Iowa, USA

${ }^{76}$ Department of Physics and Astronomy, Iowa State University, Ames, Iowa, USA

${ }^{77}$ Joint Institute for Nuclear Research, Dubna, Russia

${ }^{78 a}$ Departamento de Engenharia Elétrica, Universidade Federal de Juiz de Fora (UFJF),

Juiz de Fora, Brazil

${ }^{78 b}$ Universidade Federal do Rio De Janeiro COPPE/EE/IF, Rio de Janeiro, Brazil

${ }^{78 c}$ Universidade Federal de São João del Rei (UFSJ), São João del Rei, Brazil

${ }^{78 \mathrm{~d}}$ Instituto de Física, Universidade de São Paulo, São Paulo, Brazil

${ }^{79}$ KEK, High Energy Accelerator Research Organization, Tsukuba, Japan

${ }^{80}$ Graduate School of Science, Kobe University, Kobe, Japan

${ }^{81 \mathrm{a} A G H}$ University of Science and Technology, Faculty of Physics and Applied Computer Science, Krakow, Poland

${ }^{81 \mathrm{~b}}$ Marian Smoluchowski Institute of Physics, Jagiellonian University, Krakow, Poland

${ }^{82}$ Institute of Nuclear Physics Polish Academy of Sciences, Krakow, Poland

${ }^{83}$ Faculty of Science, Kyoto University, Kyoto, Japan

${ }^{84}$ Kyoto University of Education, Kyoto, Japan

${ }^{85}$ Research Center for Advanced Particle Physics and Department of Physics, Kyushu University, Fukuoka, Japan

${ }^{86}$ Instituto de Física La Plata, Universidad Nacional de La Plata and CONICET, La Plata, Argentina

${ }^{87}$ Physics Department, Lancaster University, Lancaster, United Kingdom

${ }^{88}$ Oliver Lodge Laboratory, University of Liverpool, Liverpool, United Kingdom

${ }^{89}$ Department of Experimental Particle Physics, Jožef Stefan Institute and Department of Physics, University of Ljubljana, Ljubljana, Slovenia

${ }^{90}$ School of Physics and Astronomy, Queen Mary University of London, London, United Kingdom

${ }^{91}$ Department of Physics, Royal Holloway University of London, Egham, United Kingdom

${ }^{92}$ Department of Physics and Astronomy, University College London, London, United Kingdom

${ }^{93}$ Louisiana Tech University, Ruston, Louisiana, USA

${ }^{94}$ Fysiska institutionen, Lunds universitet, Lund, Sweden

${ }^{95}$ Centre de Calcul de l'Institut National de Physique Nucléaire et de Physique des Particules (IN2P3), Villeurbanne, France

${ }^{96}$ Departamento de Física Teorica C-15 and CIAFF, Universidad Autónoma de Madrid, Madrid, Spain

${ }^{97}$ Institut für Physik, Universität Mainz, Mainz, Germany

${ }^{98}$ School of Physics and Astronomy, University of Manchester, Manchester, United Kingdom

${ }^{99}$ CPPM, Aix-Marseille Université, CNRS/IN2P3, Marseille, France

${ }^{100}$ Department of Physics, University of Massachusetts, Amherst, Massachusetts, USA

${ }^{101}$ Department of Physics, McGill University, Montreal, Québec, Canada

${ }^{102}$ School of Physics, University of Melbourne, Victoria, Australia

${ }^{103}$ Department of Physics, University of Michigan, Ann Arbor, Michigan, USA 
${ }^{104}$ Department of Physics and Astronomy, Michigan State University, East Lansing, Michigan, USA

${ }^{105}$ B.I. Stepanov Institute of Physics, National Academy of Sciences of Belarus, Minsk, Belarus

${ }^{106}$ Research Institute for Nuclear Problems of Byelorussian State University, Minsk, Belarus

${ }^{107}$ Group of Particle Physics, University of Montreal, Montreal, Québec, Canada

${ }^{108}$ P.N. Lebedev Physical Institute of the Russian Academy of Sciences, Moscow, Russia

${ }^{109}$ Institute for Theoretical and Experimental Physics (ITEP), Moscow, Russia

${ }^{110}$ National Research Nuclear University MEPhI, Moscow, Russia

${ }^{111}$ D.V. Skobeltsyn Institute of Nuclear Physics, M.V. Lomonosov Moscow State University, Moscow, Russia

${ }^{112}$ Fakultät für Physik, Ludwig-Maximilians-Universität München, München, Germany

${ }^{113}$ Max-Planck-Institut für Physik (Werner-Heisenberg-Institut), München, Germany

${ }^{114}$ Nagasaki Institute of Applied Science, Nagasaki, Japan

${ }^{115}$ Graduate School of Science and Kobayashi-Maskawa Institute, Nagoya University, Nagoya, Japan

${ }^{116}$ Department of Physics and Astronomy, University of New Mexico, Albuquerque, New Mexico, USA

${ }^{117}$ Institute for Mathematics, Astrophysics and Particle Physics, Radboud University Nijmegen/Nikhef, Nijmegen, Netherlands

${ }^{118}$ Nikhef National Institute for Subatomic Physics and University of Amsterdam, Amsterdam, Netherlands

${ }^{119}$ Department of Physics, Northern Illinois University, DeKalb, Illinois, USA

${ }^{120 a}$ Budker Institute of Nuclear Physics, SB RAS, Novosibirsk, Russia

${ }^{120 \mathrm{~b}}$ Novosibirsk State University Novosibirsk, Russia

${ }^{121}$ Department of Physics, New York University, New York, New York, USA

${ }^{122}$ The Ohio State University, Columbus, Ohio, USA

${ }^{123}$ Faculty of Science, Okayama University, Okayama, Japan

${ }^{124}$ Homer L. Dodge Department of Physics and Astronomy, University of Oklahoma, Norman, Oklahoma, USA

${ }^{125}$ Department of Physics, Oklahoma State University, Stillwater, Oklahoma, USA

${ }^{126}$ Palacký University, RCPTM, Joint Laboratory of Optics, Olomouc, Czech Republic

${ }^{127}$ Center for High Energy Physics, University of Oregon, Eugene, Oregon, USA

${ }^{128}$ LAL, Université Paris-Sud, CNRS/IN2P3, Université Paris-Saclay, Orsay, France

${ }^{129}$ Graduate School of Science, Osaka University, Osaka, Japan

${ }^{130}$ Department of Physics, University of Oslo, Oslo, Norway

${ }^{131}$ Department of Physics, Oxford University, Oxford, United Kingdom

${ }^{132}$ LPNHE, Sorbonne Université, Paris Diderot Sorbonne Paris Cité, CNRS/IN2P3, Paris, France

${ }^{133}$ Department of Physics, University of Pennsylvania, Philadelphia, Pennsylvania, USA

${ }^{134}$ Konstantinov Nuclear Physics Institute of National Research Centre "Kurchatov Institute", PNPI, St. Petersburg, Russia

${ }^{135}$ Department of Physics and Astronomy, University of Pittsburgh, Pittsburgh, Pennsylvania, USA

${ }^{136 a}$ Laboratório de Instrumentação e Física Experimental de Partículas-LIP, Portugal

${ }^{136 \mathrm{~b}}$ Departamento de Física, Faculdade de Ciências, Universidade de Lisboa, Lisboa, Portugal

${ }^{136 \mathrm{c}}$ Departamento de Física, Universidade de Coimbra, Coimbra, Portugal

${ }^{136 \mathrm{~d}}$ Centro de Física Nuclear da Universidade de Lisboa, Lisboa, Portugal

${ }^{136 e}$ Departamento de Física, Universidade do Minho, Braga, Portugal

${ }^{136 f}$ Departamento de Física Teorica y del Cosmos, Universidad de Granada, Granada (Spain), Spain

${ }^{136 \mathrm{~g}}$ Dep Física and CEFITEC of Faculdade de Ciências e Tecnologia, Universidade Nova de Lisboa, Caparica, Portugal

${ }^{137}$ Institute of Physics, Academy of Sciences of the Czech Republic, Prague, Czech Republic

${ }^{138}$ Czech Technical University in Prague, Prague, Czech Republic

${ }^{139}$ Charles University, Faculty of Mathematics and Physics, Prague, Czech Republic

${ }^{140}$ State Research Center Institute for High Energy Physics, NRC KI, Protvino, Russia

${ }^{141}$ Particle Physics Department, Rutherford Appleton Laboratory, Didcot, United Kingdom

${ }^{142}$ IRFU, CEA, Université Paris-Saclay, Gif-sur-Yvette, France

${ }^{143}$ Santa Cruz Institute for Particle Physics, University of California Santa Cruz, Santa Cruz, California, USA

${ }^{144 a}$ Departamento de Física, Pontificia Universidad Católica de Chile, Santiago, Chile

${ }^{144 \mathrm{~b}}$ Departamento de Física, Universidad Técnica Federico Santa María, Valparaíso, Chile

${ }^{145}$ Department of Physics, University of Washington, Seattle, Washington, USA

${ }^{146}$ Department of Physics and Astronomy, University of Sheffield, Sheffield, United Kingdom

${ }^{147}$ Department of Physics, Shinshu University, Nagano, Japan

${ }^{148}$ Department Physik, Universität Siegen, Siegen, Germany

${ }^{149}$ Department of Physics, Simon Fraser University, Burnaby, British Columbia, Canada 


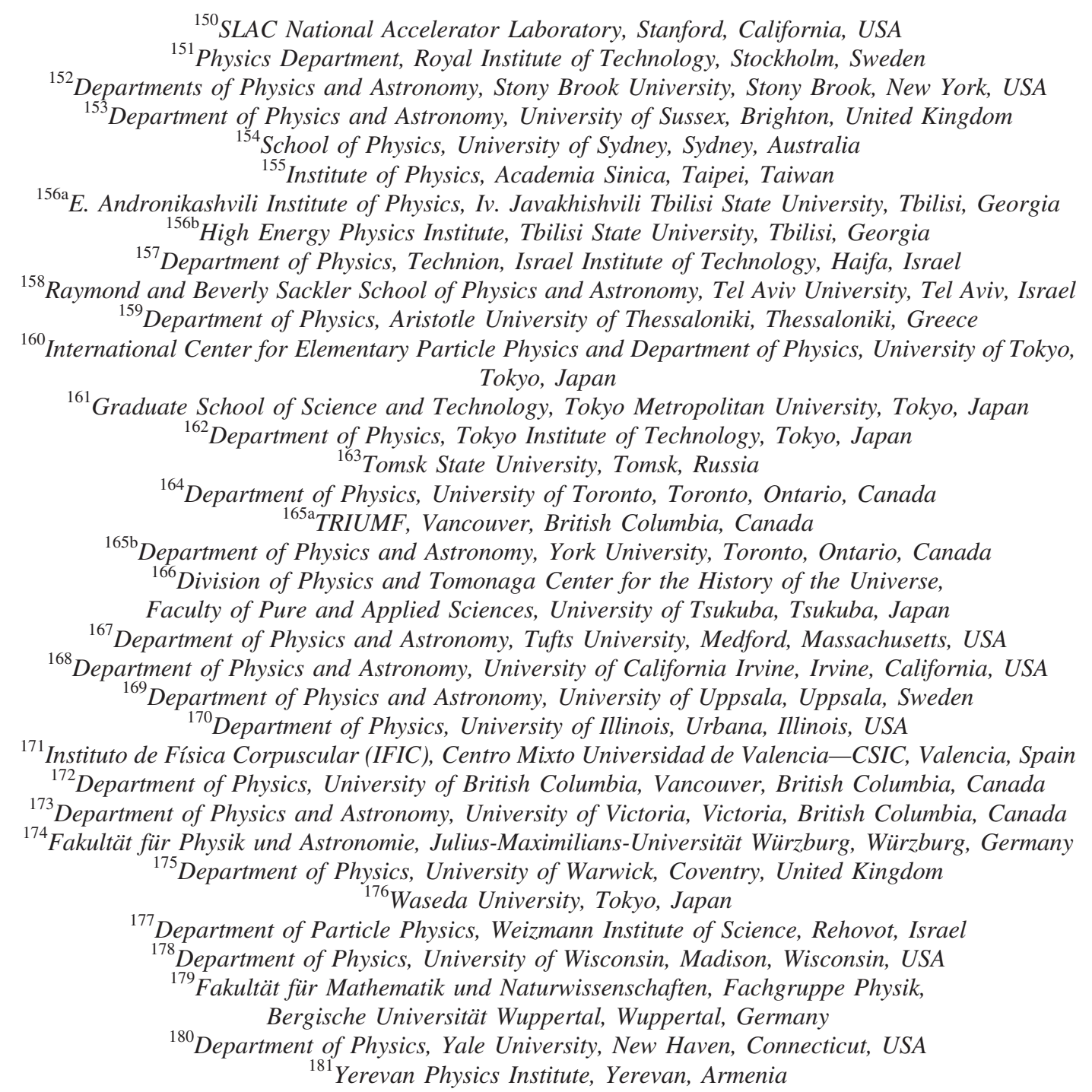

${ }^{\mathrm{a}}$ Deceased.

${ }^{\mathrm{b}}$ Also at Department of Physics, King's College London, London, United Kingdom.

${ }^{\mathrm{c}}$ Also at Istanbul University, Dept. of Physics, Istanbul, Turkey.

${ }^{\mathrm{d}}$ Also at Institute of Physics, Azerbaijan Academy of Sciences, Baku, Azerbaijan.

${ }^{\mathrm{e}}$ Also at TRIUMF, Vancouver, British Columbia, Canada.

${ }^{\mathrm{f}}$ Also at Department of Physics and Astronomy, University of Louisville, Louisville, Kentucky, USA.

${ }^{g}$ Also at Department of Physics, California State University, Fresno, California, USA.

${ }^{\mathrm{h}}$ Also at Department of Physics, University of Fribourg, Fribourg, Switzerland.

${ }^{\mathrm{i}}$ Also at Departament de Fisica de la Universitat Autonoma de Barcelona, Barcelona, Spain.

${ }^{\mathrm{j}}$ Also at Tomsk State University, Tomsk, and Moscow Institute of Physics and Technology State University, Dolgoprudny, Russia.

${ }^{\mathrm{k}}$ Also at The Collaborative Innovation Center of Quantum Matter (CICQM), Beijing, China.

${ }^{1}$ Also at Universita di Napoli Parthenope, Napoli, Italy.

${ }^{\mathrm{m}}$ Also at Institute of Particle Physics (IPP), Canada.

${ }^{\mathrm{n}}$ Also at II. Physikalisches Institut, Georg-August-Universität Göttingen, Göttingen, Germany.

${ }^{\circ}$ Also at Dipartimento di Fisica E. Fermi, Università di Pisa, Pisa, Italy.

${ }^{\mathrm{p}}$ Also at Horia Hulubei National Institute of Physics and Nuclear Engineering, Bucharest, Romania.

${ }^{\mathrm{q}}$ Also at CPPM, Aix-Marseille Université, CNRS/IN2P3, Marseille, France.

${ }^{\mathrm{r}}$ Also at Department of Physics, St. Petersburg State Polytechnical University, St. Petersburg, Russia.

${ }^{\mathrm{s}}$ Also at Borough of Manhattan Community College, City University of New York, New York, USA.

${ }^{t}$ Also at Department of Financial and Management Engineering, University of the Aegean, Chios, Greece.

${ }^{u}$ Also at Centre for High Performance Computing, CSIR Campus, Rosebank, Cape Town, South Africa.

${ }^{v}$ Also at Louisiana Tech University, Ruston, Louisiana, USA. 
${ }^{\mathrm{w}}$ Also at Institucio Catalana de Recerca i Estudis Avancats, ICREA, Barcelona, Spain.

${ }^{\mathrm{x}}$ Also at Department of Physics, University of Michigan, Ann Arbor, Michigan, USA.

${ }^{y}$ Also at LAL, Université Paris-Sud, CNRS/IN2P3, Université Paris-Saclay, Orsay, France.

${ }^{\mathrm{z}}$ Also at Graduate School of Science, Osaka University, Osaka, Japan.

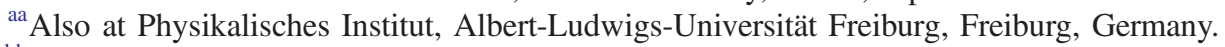

${ }^{\mathrm{bb}}$ Also at Institute for Mathematics, Astrophysics and Particle Physics, Radboud University Nijmegen/Nikhef, Nijmegen, Netherlands.

${ }^{\mathrm{cc}}$ Also at Near East University, Nicosia, North Cyprus, Mersin, Turkey.

${ }^{\mathrm{dd}}$ Also at Institute of Theoretical Physics, Ilia State University, Tbilisi, Georgia.

${ }^{\mathrm{ee}}$ Also at CERN, Geneva, Switzerland.

${ }^{\mathrm{ff}}$ Also at Department of Physics, Stanford University, Stanford, California, USA.

${ }^{g g}$ Also at Manhattan College, New York, New York, USA.

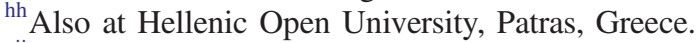

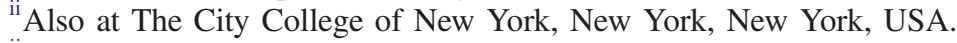

${ }^{\mathrm{jj}}$ Also at Departamento de Física Teorica y del Cosmos, Universidad de Granada, Granada (Spain), Spain.

${ }^{\mathrm{kk}}$ Also at Department of Physics, California State University, Sacramento, California, USA.

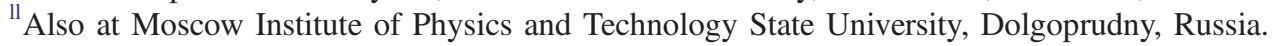

${ }^{\mathrm{mm}}$ Also at Département de Physique Nucléaire et Corpusculaire, Université de Genève, Genève, Switzerland.

${ }^{n n}$ Also at Department of Physics and Astronomy, University of Sheffield, Sheffield, United Kingdom.

${ }^{\text {oo }}$ Also at School of Physics, Sun Yat-sen University, Guangzhou, China.

${ }^{\mathrm{pp}}$ Also at Department of Applied Physics and Astronomy, University of Sharjah, Sharjah, United Arab Emirates.

${ }^{\mathrm{qq}}$ Also at Institut für Experimentalphysik, Universität Hamburg, Hamburg, Germany.

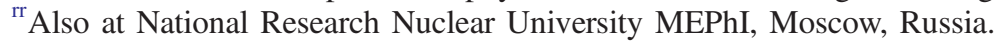

${ }^{\text {ss }}$ Also at Institute for Particle and Nuclear Physics, Wigner Research Centre for Physics, Budapest, Hungary.

${ }^{t t}$ Also at Giresun University, Faculty of Engineering, Giresun, Turkey.

${ }^{\mathrm{uu}}$ Also at Department of Physics, Nanjing University, Nanjing, China.

${ }^{v v}$ Also at Institute of Physics, Academia Sinica, Taipei, Taiwan.

${ }^{\text {ww }}$ Also at Department of Physics, University of Malaya, Kuala Lumpur, Malaysia. 